\title{
Image and Color Stylization through Region-based Abstraction
}

by

Rosa Azami

A thesis submitted to the Faculty of Graduate and Postdoctoral Affairs in partial fulfillment of the requirements for the degree of

\section{Doctor of Philosophy in} Computer Science

Ottawa-Carleton Institute for Computer Science School of Computer Science

Carleton University

Ottawa, Ontario

May, 2021

(C)Copyright

Rosa Azami, 2021 


\section{Abstract}

In Non-Photorealistic Rendering (NPR), abstraction methods simplify images. The NPR techniques produce stylized imagery by emphasizing the main structures through stroke elements, colors and removing unessential image information. However, the difficulty of abstraction lies in deciding what is important to be presented. This thesis introduces a few non-photorealistic rendering techniques for stylizing images by manipulating the image's elements such as details, complex region forms, and colors. To increase the visual interest in abstractions, it integrates contrast, texture, and structure preservation in designing the proposed algorithms.

We propose an abstraction method based on an over-segmentation through overlapping region growth. The main idea of this method is to create stylized images by complex primitives. Unlike traditional over-segmentation methods, this method emphasizes the irregularity of region shape even in uniform areas. The region creation by overlapping allows us to preserve small structures. Additionally, we extend the creation of stylized images through this framework by manipulating the color and shapes of the region primitives. As an application of the region-based framework, we present a novel recoloring algorithm based on a tree-based structure, which produces wild recolorizations. A set of colorful abstractions are produced with various styles by considering contrast and structure preservation.

We further engage image regions in providing a method for improving stippling in extreme-tone regions of the image. This method identifies dark regions and replaces them with polygons rather than stipples. For light regions, it suggests a mechanism to control the stipple placement. This approach generates stippled images with better visual quality than the originals despite using fewer stipples.

Finally, we present an automatic system for photo manipulation that brightens an abstraction and alters the detail levels. By employing a smooth map, we brighten the photograph such that the areas of interest will get increased contrast while colors in the areas of less interest will become lighter and less saturated. We define a system for scoring texture elements, to establish different levels of texture indication. 
to my parents 


\section{Acknowledgments}

Prof. David Mould has mentored me and given me the opportunity to work in this exciting field of research, and I am very grateful for his guidance, patience and motivation. Without his guidance and tremendous help, I wouldn't be able to finish this thesis.

I'd like to thank the rest of my thesis committee, Prof. Oliver van Kaick from Carleton University, Prof. Jochen Lang from the University of Ottawa, Prof. Craig S. Kaplan from the University of Waterloo, and Prof. Richard Dansereau from Carleton University, for their insightful comments and discussions on the content of my research.

Further, I thank my fellow labemates, the members of the Graphics, Imaging, and Games Lab in the School of Computer Science at Carleton University. I am grateful for having the chance to work in a friendly environment in the GIGL lab. Studying abroad had its own ups and downs and I am glad I have made friends among colleagues that made my life easier while being far from family.

Finally, I would like to thank my family for their love and support along this journey. I would like to thank Omid Sakhi, my dear friend who has also been there to support me in difficult times. 


\section{Preface}

Versions of Chapters 3, 4, 5, and 6 have been published in the following papers:

- Rosa Azami and David Mould. "Image abstraction through overlapping region growth," in Proceedings of Graphics Interface 2020, Canadian Human-Computer Communications Society / Société canadienne du dialogue humain-machine, 2020, pp. $66-73$.

- Rosa Azami and David Mould. "Artistic Recoloring of Image Oversegmentations," in Proceedings of Graphics Interface 2021, Canadian Human-Computer Communications Society / Société canadienne du dialogue humain-machine.

- Rosa Azami, Lars Doyle, and David Mould. "Stipple Removal in Extreme-tone Regions," In Proceedings of the 8th ACM/Eurographics Expressive Symposium on Computational Aesthetics and Sketch Based Interfaces and Modeling and Non-Photorealistic Animation and Rendering (Expressive '19). Eurographics Association, Goslar, DEU, 2019, pp. 123-132.

- Rosa Azami and David Mould. "Detail and Color Enhancement in Photo Stylization," In Proceedings of the symposium on Computational Aesthetics (CAE 17). Association for Computing Machinery, New York, NY, USA, Article 5, 2017, pp. 1-11.

The ideas in this thesis originated in discussions between myself and my supervisor, David Mould. I conducted the implementation, testing, and contributed to writing the manuscripts. David Mould provided guidance, intuitions, and contributed to the writing of the manuscripts.

Lars Doyle contributed to writing the paper from chapter 5 . He also provided the implementation of Poisson reconstruction used for the results. 


\section{Table of Contents}

$\begin{array}{lll}\text { Abstract } & \text { ii }\end{array}$

Acknowledgments $\quad$ iv

Preface $\quad$ v

List of Tables $\quad$ ix

List of Figures $\quad x$

1 Introduction 1

1.1 Problem Statement . . . . . . . . . . . . . . . . . 6

1.2 Contributions and Thesis Structure $\ldots \ldots \ldots \ldots$

1.3 Summary . . . . . . . . . . . . . . . . . . . 11

2 Literature Review 12

2.1 Abstraction Methods in NPR . . . . . . . . . . . . . . 12

2.1.1 Region-based Methods . . . . . . . . . . . . . . . . . 12

2.1.2 Filter-based Methods . . . . . . . . . . . . . . . 15

2.1.3 Stroke-based Methods . . . . . . . . . . . . . . . . . 16

2.1.4 Example-based Methods . . . . . . . . . . . . . . . . 17

2.2 Detail Enhancement Methods . . . . . . . . . . . . . . . . . 18

2.3 Color Palettes and Recoloring Methods . . . . . . . . . . . . . . 19

2.3.1 Example-based Recoloring . . . . . . . . . . . . . . . . . . 19

2.3.2 Palette-based Recoloring . . . . . . . . . . . . . . 20

2.4 Evaluation . . . . . . . . . . . . . . . . . . . . . . . . 21

2.5 Fundamental Material . . . . . . . . . . . . . . . . . . . . . 23

3 Region-based Abstraction $\quad 26$

3.1 Introduction . . . . . . . . . . . . . . . . . 26

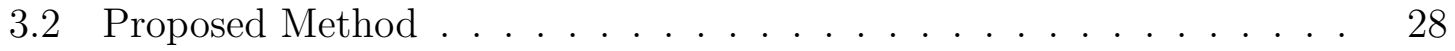


3.2 .1 Seed Placement . . . . . . . . . . . . . . . . . . . . . . . 29

3.2 .2 Growth Mechanism . . . . . . . . . . . . . . . . . . 32

3.2 .3 Rendering . . . . . . . . . . . . . . . . . . 33

3.3 Reshaping Region Boundaries . . . . . . . . . . . . . . . . 36

3.4 Results and Discussion . . . . . . . . . . . . . . . . . . 38

3.4.1 Comparison with Oversegmentation Methods . . . . . . . . 42

3.4.2 Reduced Color Palettes . . . . . . . . . . . . . . . . . . . 42

3.4.3 Performance . . . . . . . . . . . . . . . . . . . . . . . 44

3.4.4 Limitations . . . . . . . . . . . . . . . . . . . . . . . . 44

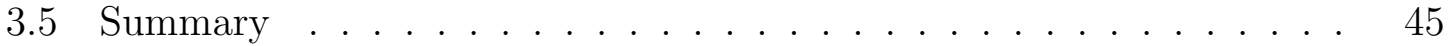

4 Recoloring of Region-based Abstraction 46

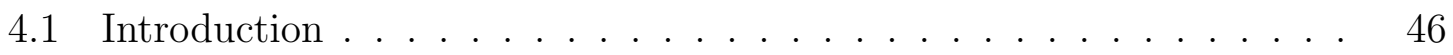

4.2 Recoloring Algorithm . . . . . . . . . . . . . . . . . . . . 48

4.2.1 Tree Creation from Widest Path . . . . . . . . . . . . . . 49

4.2.2 Tree Creation: The Widest Path Problem . . . . . . . . . 50

4.2 .3 Histogram Matching . . . . . . . . . . . . . . . . . . 52

4.2 .4 Color Assignment . . . . . . . . . . . . . . . . . . . . . . 53

4.3 Results and Discussion . . . . . . . . . . . . . . . 55

4.3 .1 Comparison with Naïve Methods . . . . . . . . . . . . . 59

4.3.2 Comparison with ColorArt . . . . . . . . . . . . . 61

4.3.3 Recoloring with SLIC0 Oversegmentation . . . . . . . . . . 61

4.3 .4 Performance . . . . . . . . . . . . . . . . . . . 61

4.3 .5 Limitations . . . . . . . . . . . . . . . . . . . . 63

4.4 Variations . . . . . . . . . . . . . . . . . . . . . 64

4.4 .1 Blending . . . . . . . . . . . . . . . . . . 65

4.4.2 Color Spaces and Distance Metrics _. . . . . . . . . . 65

4.4 .3 Reducing Palette Size . . . . . . . . . . . . . . . . . 68

4.5 Summary . . . . . . . . . . . . . . . . . . . . 69

5 Improving Stippling in Extreme-tone Regions $\quad 70$

5.1 Introduction . . . . . . . . . . . . . . . . 70

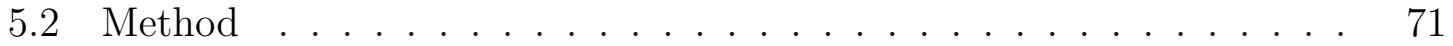

$5.2 .1 \quad$ Illustrating Dark Regions . . . . . . . . . . . . . . . 73

5.2 .2 Stipple Removal in Light Regions . . . . . . . . . . . . . 75 
5.2 .3 Detail Enhancement _. . . . . . . . . . . . . . . . . . . 78

5.3 Results and Discussion . . . . . . . . . . . . . . . . . 78

5.3.1 Visual Evaluation . . . . . . . . . . . . . . . . . . 79

5.3.2 Performance . . . . . . . . . . . . . . . . . . . . . 82

5.3 .3 Limitations ................... . . . 83

5.4 Summary . . . . . . . . . . . . . . . . 86

6 Hybrid Detail Enhancement and Abstraction $\quad 87$

6.1 Introduction . . . . . . . . . . . . . . . . . . . . . 87

6.2 Poisson Equation . . . . . . . . . . . . . . . . . . . 88

6.3 Methodology . . . . . . . . . . . . . . . . . . . 89

6.3.1 Sticks Filtering . . . . . . . . . . . . . . . 90

6.3.2 Scoring Connected Components . . . . . . . . . . . . . . 91

6.3.3 Poisson Solver . . . . . . . . . . . . . . . . . . . . 92

6.4 Hybrid Abstraction . . . . . . . . . . . . . . . . . . 93

6.4.1 Image Brightening . . . . . . . . . . . . . . . . 93

6.4.2 Detail Enhancement . . . . . . . . . . . . . . . . . . 94

6.4.3 Effect of Parameters . . . . . . . . . . . . . . . . . . . . . . . 99

6.4.4 Comparisons ................... 101

6.4.5 Timing....................... 103

6.4.6 Limitations . . . . . . . . . . . . . . . 103

6.5 Summary . . . . . . . . . . . . . . . . . 104

7 Conclusions and Future Work 106

7.1 Conclusions . . . . . . . . . . . . . . . . 107

7.2 Future Work . . . . . . . . . . . . . . . . . . . . 108

$\begin{array}{ll}\text { List of References } & 111\end{array}$

$\begin{array}{ll}\text { Appendix A Image Copyright } & 122\end{array}$ 


\section{List of Tables}

4.1 Timing results for images with varying numbers of regions. . . . . . 63

5.1 Stipple and polygon counts from SPS before and after applying our

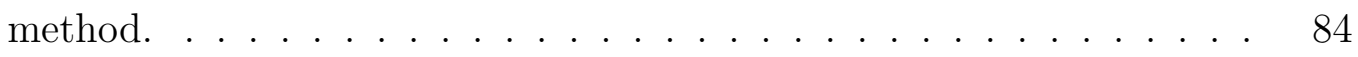

5.2 Stipple counts from LBG stippling before and after applying our method. 84 


\section{List of Figures}

1.1 Left: an abstraction art by Franz Cline, which used a few basic strokes to draw a face. Right: abstraction of Eiffel Tower by Robert Delaunay by combination of rectangles and small triangles. . . . . . . . . .

1.2 Examples of stylization methods in NPR. From left to right and top to bottom: anisotropic kuwahara filtering [74], structured abstraction [43], artistic thresholding [140] and stippling technique [31]. . . .

1.3 An example of the indication/detail technique [137]. The house is drawn with full detail in the upper left corner. In the house on the lower left corner, line segments drawn by a user, show where the most detail was desired. The final sketch on the right has varied levels of detail. . . . . . . . . . . . . . . . .

1.4 Examples of Fauvism art. Pont de Charing Cross by André Derain and Portrait of Madame Matisse The Green Line by Henri Matisse . .

1.5 The filtered image of the lion [74] has lost details on the whiskers, fur, and the eye pupil. The original photograph shown on the left is courtesy of Tambako the Jaguar. . . . . . . . . . . . . . . . . .

3.1 Abstraction result. Left to right: input image, modal region colors, simplified boundaries, black and white. . . . . . . . . . . . 27

3.2 Irregular regions generated by our method. . . . . . . . . . . . . . 28

3.3 Region-based abstraction stages. We compute a detail layer from input image and its filtered one. Then, we generate initial seeds for each region from the connected components of this detail map. For each seed, we expand a region. When all regions have been found, we color the output by assigning to each region the mode of the pixel values in the visible portion of that region. . . . . . . . . . . . . . . . 29

3.4 Left to right: absolute residuals vs positive/negative residuals. . . . . 31 
3.5 Seed placement. Left: all connected components of the (negative) detail layer in owl image. Right: close-up of the rectangle area on the left image, showing the selected seeds for each connected components of the detail layer. . . . . . . . . . . . . . . . . . .

3.6 Residuals divided into positive and negative connected components (color coded) have a close relation with the image content. . . . . . .

3.7 Examples of regions generated by the free-growth mechanism. The red square on the image illustrates several paths from seed to pixels terminating the region's growth.

3.8 Flattening. Left to right: overlapped regions, the contours after flattening process and the rendered image. . . . . . . . . . . . . . 34

3.9 Region boundaries in stump image. . . . . . . . . . . . . . . . . 35

3.10 An image of a noisy texture used as background texture for the sky in the arch image. . . . . . . . . . . . . . . . .

3.11 Abstractions after boundary simplification by Ramer-Douglas-Peucker Algorithm $(\epsilon=4)$, with initially generated regions (middle) and with flattened regions (right). . . . . . . . . . . . . .

3.12 Regions simplified with boundary smoothing using Fourier shape descriptors. . . . . . . . . . . . . . . . .

3.13 Image abstraction results. Left to right: the original images, abstractions with flattened regions and regions with boundary simplification using RDP algorithm [36]. Top to bottom: market, temple, autumn,

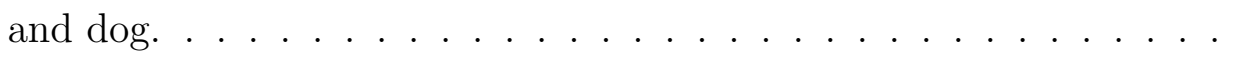

3.14 Image abstraction results (set2). Left to right: the original images, contours after flattening, abstractions with flattened regions and regions with boundary simplification using RDP algorithm [36]. Top to bottom: eye, lizard, parrots, and mountains. . . . . . . . . . . .

3.15 Original images of stump, port, bridge, owl, man, arch, old man, tomatoes, toque, and machinery. . . . . . . . . . . . .

3.16 Region comparison. Left to right: regions from SLIC [3], QuickShift [131], Felzenszwalb [45], and our method. . . . . . . . . . . 43

3.17 Cat and port in black and white. . . . . . . . . . . . . . 43 
3.18 Recoloring using a color palette from Art Deco Poster. Left to right: Art Deco poster and extracted color palette; recolored old man; and parrot. . . . . . . . . . . . . . . .

3.19 Uninteresting region shapes in the blurred background of toque and smooth surfaces on tomatoes. . . . . . . . . . . . . .

4.1 Colorful representational images. A modern vector illustration of a lion; a Fauvist painting by André Derain; an abstraction of the Eiffel Tower by Robert Delaunay. . . . . . . . . . . . . . . . . . . . . . . . 47

4.2 Recoloring algorithm pipeline. . . . . . . . . . . . . . . . . 50

4.3 Histogram matching color distances: cdfs of the original image $\{\Delta Q\}$ (blue), the palette $\{\Delta P\}$ (red), and the target color differences $\left\{\Delta q^{\prime}\right\}$ (yellow) . . . . . . . . . . . . . . . . .

4.4 Histogram matching result. Left to right: original image, result without histogram matching, and result using histogram matching. . .

4.5 Changing the source region locations. The starting region is indicated by a dot. . . . . . . . . . . . . . . . . . . .

4.6 Region recoloring results. Images, top to bottom: starfish, Venice, and lizard. . . . . . . . . . . . . . . 56

4.7 Region recoloring results. Images: lanterns and rust. . . . . . . . . . 57

4.8 A comparison with naïve recolorings. Left to right: The original image, the results from naïve method, random recoloring, and ours. . . . . .

4.9 Comparing with naïve recoloring. Left: closest color assigned to regions, right: our results. . . . . . . . . . . . . . . . 60

4.10 Comparison with ColorArt. Left: input; middle: our results; right: ColorArt results. . . . . . . . . . . . . . . . . . . . . 62

4.11 Recoloring with SLIC0 oversegmentation. . . . . . . . . . . . 62

4.12 Failure cases. . . . . . . . . . . . . . . . . . . . . . . 64

4.13 Postprocessing with an edge-aware filter. Left to right: original image; cross-filtered images with mask size 20, 100, and 300 . . . . . . . . 66

4.14 Colorization with different distance measures. . . . . . . . . . . . . 67

4.15 Reduced color palettes. Left to right, palette sizes are 10, 8, 6, 4, and 2. 68 
5.1 Steps of our method: $a$ ) original image; b) threshold mask; $c$ ) reconstructed image after Poisson blending; $d$ ) results from filling polygons; e) final result after stipple removal; $f$ ) close-ups of original stippling and final result of the polygon filling followed by stipple removal steps. 72

5.2 Transition from a dark region to its surroundings. The graph shows the original intensity profile (star), the thresholded values (square), and the results of blending (circle). On the right, the top images show the grayscale image and corresponding smooth transition of stipples and the bottom images are the results of hard thresholding. . . . . . 74

5.3 Left: thresholded regions; right: stippled image with dark regions covered by polygons. . . . . . . . . . . . . . . . . . .

5.4 Selected details of the car and New Jersey images. On the top row, note the unnecessary stipples in the sky above the car and in the background behind the kid. On the bottom row, the unwanted stipples are removed from the background.

5.5 Stipple removal process. From left to right: result before stipple removal; stipples with 0 or 1 neighbours removed; final result after removing stipples with fewer than $c$ neighbours. . . . . . . . . . . . . . 77

5.6 Effect of detail enhancement. From left to right: original gray image; positive residuals; original structure-aware stippling; stippling after detail enhancement. . . . . . . . . . . . . . . . . . . .

5.7 Smooth transitions and pattern preservation. Left column: original stipplings; middle: our results; right: details, in which we see smooth transitions from dark to light regions and preservation of facial features in toque, and the kid's face and tattoo in New Jersey. . . . . . . . . .

5.8 Results after applying our method on parrot and headlight based on structure-preserving stippling [78]. Left: original stipplings; right: our results. . . . . . . . . . . . . . . .

5.9 Results of detail enhancement. Left: original SPS; right: detail enhancement. Red circles show particular cases of detail enhancement and the blue circle shows stipple removal. Zoom in to see details. . .

5.10 LBG stippling with our algorithm on Theater and Oparara images. Left column: original LBG stippling and right column: LBG stippling after applying our method. 
5.11 Effect of parameter $c$ in bright images. Left to right: original stippling [78], result with $c=3, c=4$, and $c=5 . \ldots . . . . .285$

6.1 An example of distance transform map (left) and its corresponding smooth map obtained from solving a Poisson equation (right). . . . .

6.2 Overview of our method. Original rock arch image (C) Vadim Kurland, CC BY 2.0. . . . . . . . . . . . . . . . . . . . . . . . . . 89

6.3 The eight masks used for sticks filtering. . . . . . . . . . . . . . . 91

6.4 Scored connected components. Left: positive residuals; right: negative residuals. . . . . . . . . . . . . . . . . . . . .

6.5 Effect of parameter $m$ in $\lambda(x, y)$. Left to right: detail maps for $m=3$, $m=4, m=5$, and $m=10 \ldots \ldots \ldots$

6.6 Example steps of the enhancement process. Left to right: original image; filtered image; result from brightening the filtered image; detail map; and the final result from merging the brightened image and the detail map. Original Angry cat CPeter Pham, CC BY 2.0. . . . . .

6.7 Brightened and detail-enhanced images from benchmark set [97] . Left to right: barn, Yemeni, desert and cat. . . . . . . . . . . . . . . . 95

6.8 Stylization effects. From top to bottom: rock arch, whale, swans and old lady. . . . . . . . . . . . . . . . . . . . . . . . . . . . . . 97

6.9 Stylization effects (continue). From top to bottom: eye, skull rocks, Venice, rock formation and waterfall. . . . . . . . . . . . . . .

6.10 Different brightness. Original Oparara image (left) [97], our results with $b=1$ (middle) and $b=2$ (right).

6.11 Detail enhancement with different thresholding of the sorted connected component scores. Left to right: original, $s_{t}=0.9, s_{t}=0.75, s_{t}=0.33$ and $s_{t}=0.16$. Original cow image (C)Alastair Campbell, CC BY-SA 2.0.100

6.12 Different exaggeration parameters. Left to right: $k=3, k=5, k=7$. Original stones image by CanyonlandsNPS (public domain) and angry cat image (CPeter Pham, CC BY 2.0. . . . . . . . . . . . . . . . . 100

6.13 Brightness comparison. Left to right: original image of Memorial Church, contrast enhancement of Lee et al. (LDR), gradient domain HDR compression of Fattal et al., our result. Original Stanford Memorial Church image (C)Paul Debevec. 
6.14 Comparison of detail enhancement. Top row, left image: original image; middle: local Laplacian filter of Paris et al.; right: our result. The bottom row shows a close-up of the wall. Original Belgium House image CRaanan Fattal. . . . . . . . . . . . . . . . . . . . . . . 102

6.15 Failure cases. Strong color shifts of sky in Venice; presence of many textures in Etretat hiding the abstract background; noise effect in Chinese man next to the edges. . . . . . . . . . . . . . . . . . . . . . . . 104

6.16 Original Flickr images used in this chapter. From upper left: stones by CanyonlandsNPS (public domain), northern cardinal (C)Bill Damon, CC BY 2.0, Chinese (C)Anja Disseldorp, CC BY 2.0, Etretat (C)Cristian Bortes, CC BY 2.0 and . . . . . . . . . . . . . . . . . . . 104

A.1 Benchmark images used in this thesis. Left to right: sicknotepix (toque), Greg Myers (tomatoes), trevorklatko (Oparara), MrClean1982 (barn), Charles Roffey (desert), Theen Moy (cat), Rob Schneider (city), James Marvin Phelps (arch), Paul Stevenson (rim lighting), Martin Kenney (Mac), Richard Messenger (Yemeni), Photos By Clark (headlight). . . . . . . . . . . . . . . . . . . . 122

A.2 Images from the Berkeley Segmentation Dataset. . . . . . . . . . . . . 123

A.3 Tambako the Jaguar (lion), Mark \& Lesley (owl), Nicholas A. Tonelli (autumn), Carl Mueller (machinery), Emi Jane (eye), Alec Gamoff (mountain), Mongkhon Pookpun (stump), David Taylor (port), Carsten Ullrich (bridge), Michal Ščuglík (dog). . . . . . . . . . 123

A.4 Vector images: leaf, sketch and lion. Market courtesy of Rosa Azami 


\section{Chapter 1}

\section{Introduction}

Non-Photorealistic Rendering (NPR) methods in computer graphics generate styl-

ized images. NPR researchers have often been inspired by traditional art, especially representational art that gives the impression of a real scene. NPR researchers have introduced abstraction methods that focus on simplifying image content $[30,50,64]$. Abstraction methods have been used to stylize images, emphasizing the main structures through stroke elements and colors and removing unessential image information. A stylized image has a new look while its content has been modified into an artistic image as if created by a traditional artistic technique, like painting, drawing, stippling, or illustration. Although traditional art has inspired many NPR methods, abstract art received less attention.

Abstract realism makes an abstract representation of a scene by taking the objects and exaggerating or simplifying them. Abstract realism combines two contradictory terms, Abstract art and Realistic art. "The concept can involve edges or it might use color and value with extreme conservation. It could embody the simplicity of shapes or rich color relationships" [1]. This way artists could emphasize some elements of the scene in their art to express their feelings or draw attention to a subject.

Artists often present the key elements of the image in abstraction. Such abstractions allow you to see the image content without conscious effort. Abstraction preserves the significant portions of the image, which are usually the image's structural features. Some structural elements of the image, such as edges, may be unimportant on their own, but they could be the result of object interactions, and preserving them helps with understanding the objects and their relationships in the image. For example, take the work of Franz Cline and Robert Delaunay in Figure 1.1. The artists 
express the important elements by using a few diagonal brush strokes or geometric structures in the creation of the face or tower, respectively. The artist removes unimportant elements from the abstraction. Unnecessary image components do not contribute to image understanding and can distract the viewer's attention; removing them has no effect on the image's core theme. Artists avoid using strokes on the areas that show small details. However, if an artist wants the viewer's attention drawn to specific components, such as little branches of a tree, he will not hesitate to add smaller strokes or regions to the abstraction.

Figure 1.2 shows examples of NPR images where algorithms are employed to simulate traditional media or simplify an image to an illustrative form. On the top left, the Kuwahara filter [74] created a paint-like effect, and on the top right, a structured abstraction method [43] changed the image into a paper-cut effect. A black and white effect appears on the bottom left, which is a result of the artistic thresholding [140] algorithm. A rendering of the traditional stippling technique [31] is on the bottom right. The NPR abstractions usually preserve useful features of the original images by using stroke elements or colors and remove the unimportant elements. However, they are less detailed and many key elements of the scene are removed. In this research, we aim to generate stylized abstractions that preserve the details and textures.

In this thesis, we focus on image-based stylizations, where we take a photograph as input and convert it to a stylized image through different rendering techniques. In general, NPR methods can be extended to the field of 3D graphics [117] and games $[90]$.

We regarded the key components such as fine detail, strong structures, and colors as the main factors of the artistic rendering. An abstraction can express the important information about the shape of the objects in a photograph by suggesting some highly detailed structures. Such manipulation of the image can transfer a dull photograph into an exciting artwork. For example, artists put small strokes and lines on thin features such as the whiskers of a cat to better characterize the cat's face. This technique is called indication [49], and gives the impression of texture without drawing every single stroke. Figure 1.3 shows an example of the indication/detail technique by Winkenbach and Salesin [137]. 

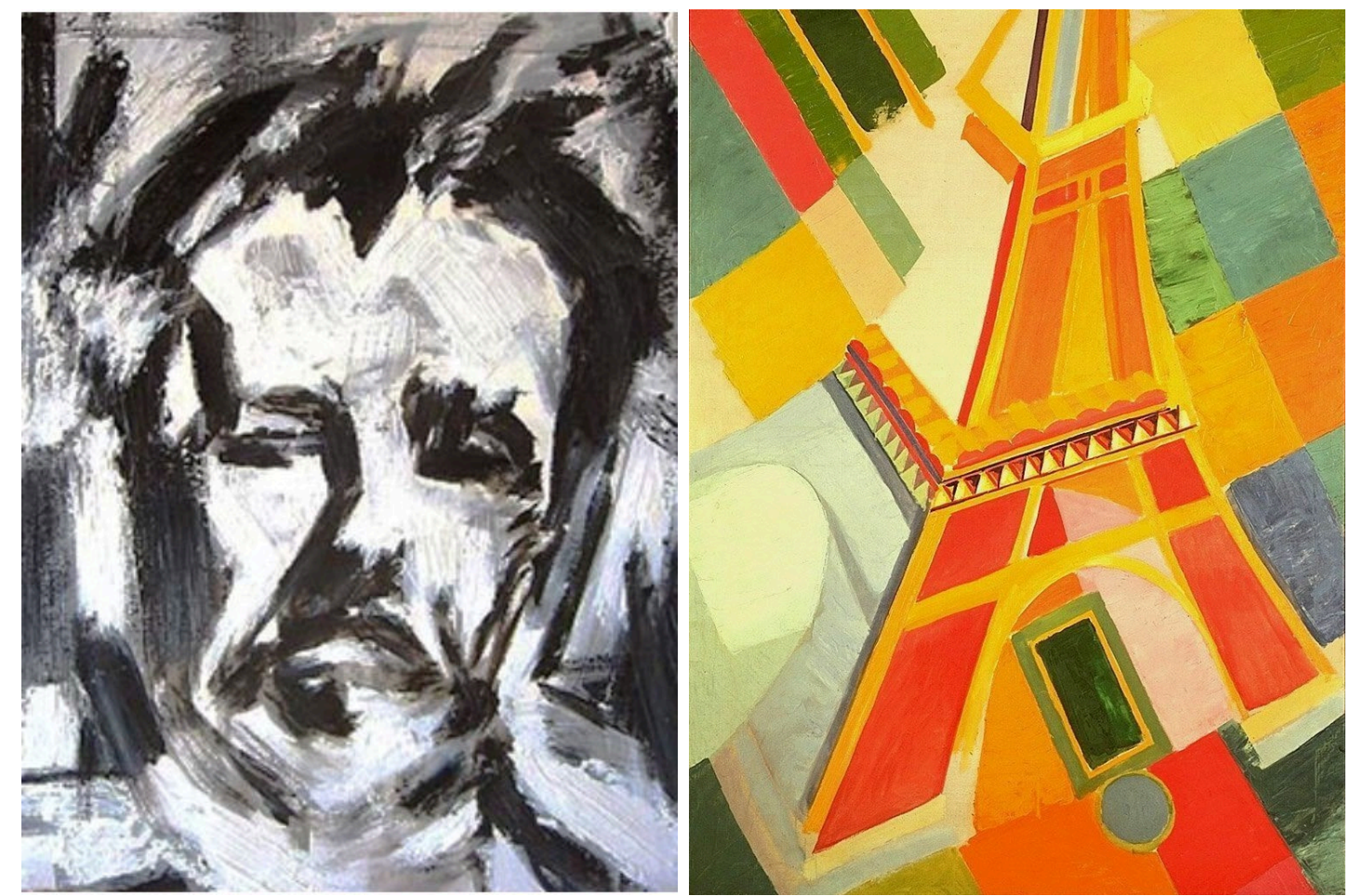

Figure 1.1: Left: an abstraction art by Franz Cline, which used a few basic strokes to draw a face. Right: abstraction of Eiffel Tower by Robert Delaunay by combination of rectangles and small triangles.

Artists also use segments for the abstraction of the image to show the main structures. In Figure 1.1, the artist used geometric structures to abstract the Eiffel tower. Segmentation methods have been used widely in NPR to extract structure from images. One way of showing an abstracted image could be combinations of different scale segments, possibly overlapping. For the purpose of abstraction, researchers $[30,43,143]$ obtained hierarchies of regions by performing the segmentation process at many different scales and constructed a tree to relate the segmented regions from different scales. An interactive abstract painting system called Sisley [143], decomposes an image into a hierarchy of its regions and applies random shifts to change the region's colors. Through a painterly rendering, the region boundaries can deviate with respect to a desired abstract level. In our research, we will investigate different styles by manipulating the shape and color of the objects.

In general, NPR methods can be classified into four categories [72]:

Stroke-based methods which approximate the image using a primitive other than colored pixels. Techniques such as stippling [23,67,77, 78, 88, 92, 103] use 

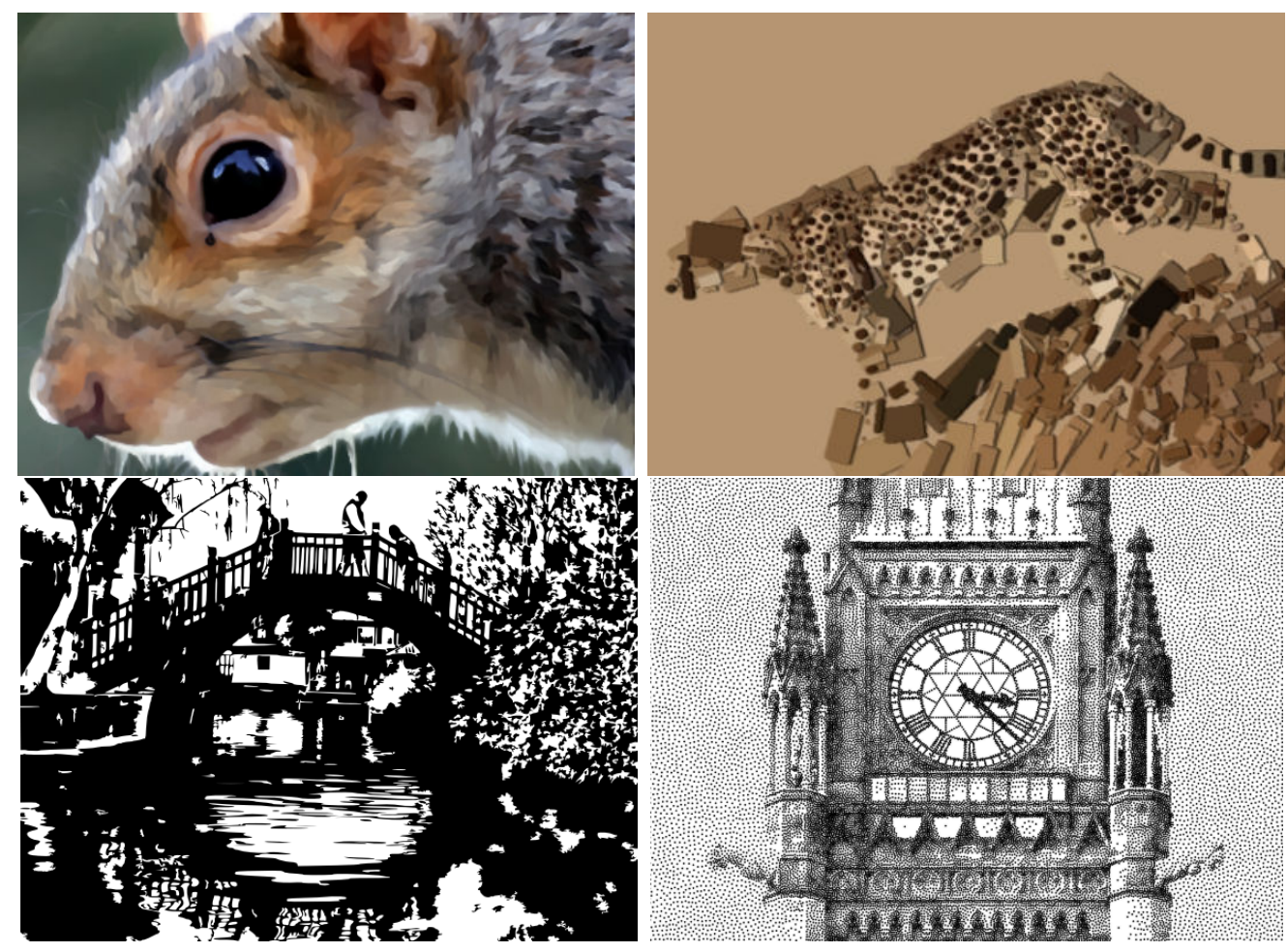

Figure 1.2: Examples of stylization methods in NPR. From left to right and top to bottom: anisotropic kuwahara filtering [74], structured abstraction [43], artistic thresholding [140] and stippling technique [31].

small dots to represent the regions with different tone distributions. Other techniques such as hatching $[12,61,62]$ and mosaicing $[52,66,82,100]$ reproduce artistic images using line/curve segments and small tiles, respectively.

The other group of techniques rely on region-based renderings, which apply segmentation to extract the image structure, then use regions as rendering primitives for creating artistic styles [30,37, 91, 122, 125, 140, 143].

Example-based methods are modeled to transfer either texture or color from a target image to a reference image $[12,55,61,62,67,87,88,126]$. Advances in machine learning algorithms produced images with complex content transfers and creativity through combining visual content and style [24,46, 79, 80, 135].

Most filter-based methods conducted a smoothing process or edge-preservation rather than stylizing the image. According to Kyprianidis et al. [72] most filtering efforts have been undertaken outside of image stylization and not many 


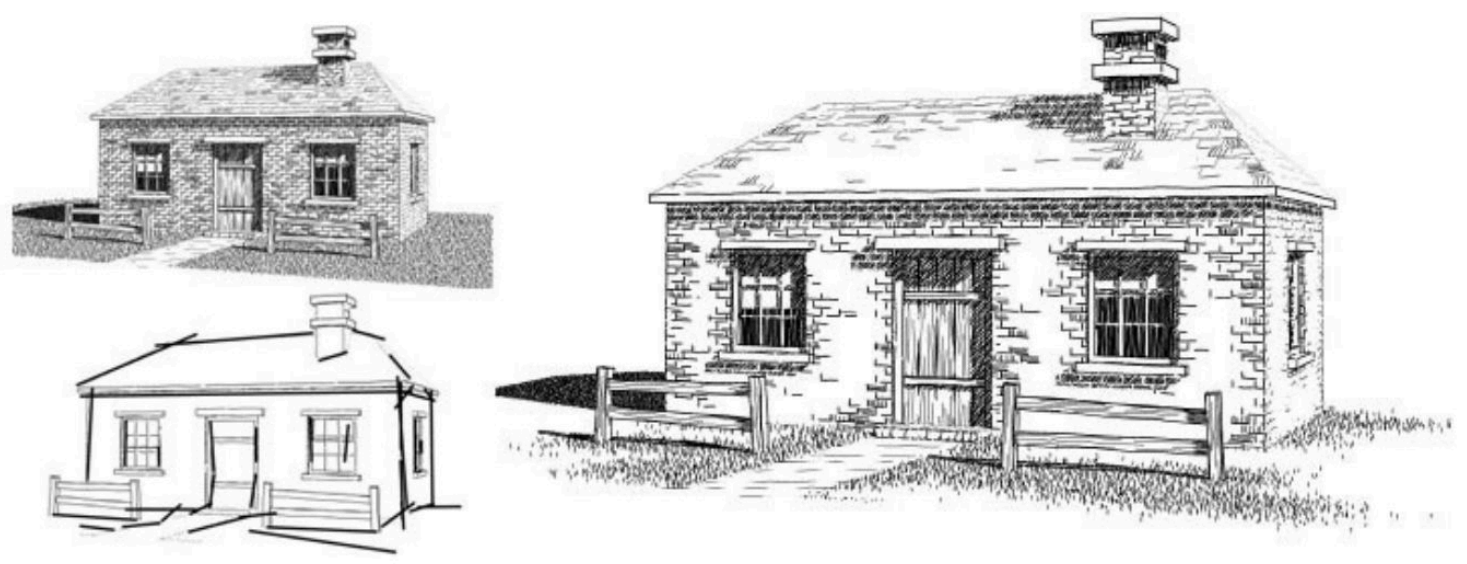

Figure 1.3: An example of the indication/detail technique [137]. The house is drawn with full detail in the upper left corner. In the house on the lower left corner, line segments drawn by a user, show where the most detail was desired. The final sketch on the right has varied levels of detail.

filter-based methods $[71,74,94,101,104]$ contributed to non-photorealistic rendering.

In our research, we want to create different image stylizations, such as black/white effect, recoloring, boundary deformation, and stippling. In the creation of stylized images, we aim to maintain several principles. We want to emulate the practice of artists, who paint only the notable detail of a scene while eliminating irrelevant detail. In addition to indicating the perceptually important details, we want our stylizations to keep the main structures intact and preserve strong edges.

A broad range of image manipulation is possible from abstractions in the form of regions. For example, a stylization generated by a region-based abstraction could resemble Fauvism, where the use of color and form translate the emotions into objects in paintings. Typically for Fauvism, the strong brush strokes use paint directly from the palette instead of preparing and blending it. The bold coloring of the objects in the painting makes them recognizable from the background. Figure 1.4 shows examples of Fauvism paintings of Pont de Charing Cross by André Derain and Portrait of Madame Matisse The Green Line by Henri Matisse. Artists made a clear separation of regions by assigning different colors to the neighboring regions.

Techniques such as stippling have been adapted with good tone and structure preservation $[23,78,103]$; while, most studies had focus on the tone matching in this area, presenting the extreme-tone regions had less attention. Regions that have no 

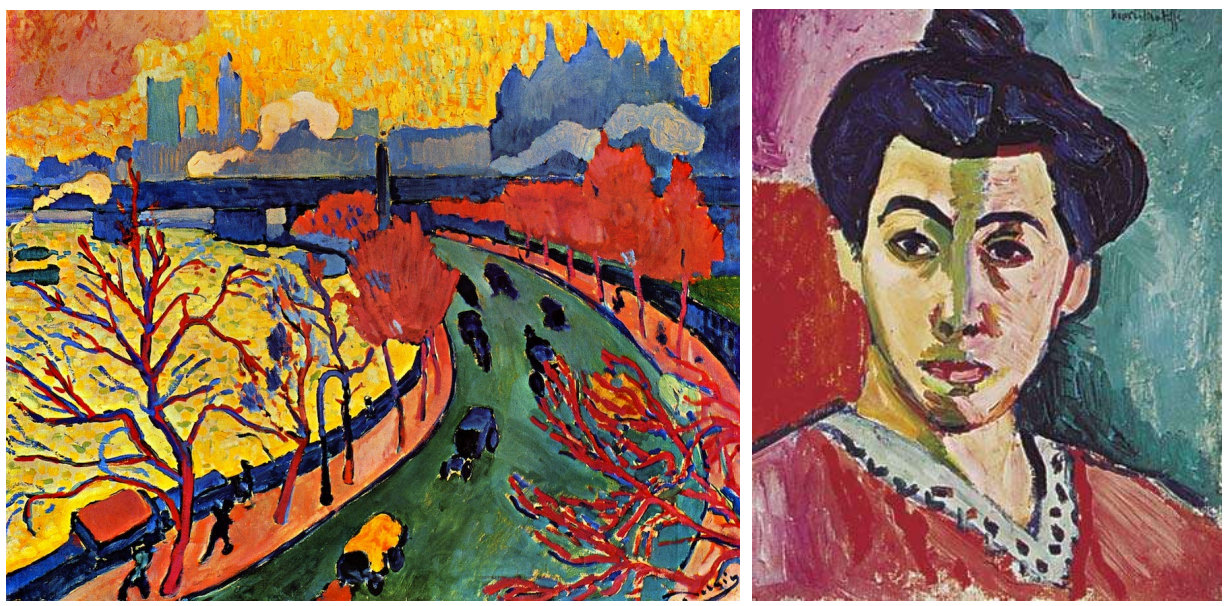

Figure 1.4: Examples of Fauvism art. Pont de Charing Cross by André Derain and Portrait of Madame Matisse The Green Line by Henri Matisse .

texture and are very light, still receive unwanted stipples, and dark regions are piled up with stipples, showing unpleasant gaps in between stipples. Treating the extremetone areas of the image can improve the stippling presentation.

\section{$1.1 \quad$ Problem Statement}

We address several problems that appear in Non-Photorealistic Rendering and describe the importance of treatment for each problem.

1. Loss of details and small-scale structures. Abstraction methods in NPR often remove details from image content; loss of such details can reduce the aesthetics of the abstracted image. This can happen when a global function (e.g. smoothing filter) changes the image smoothness/sharpness. Abstraction methods often simplify images by removing high-frequency details. Losing the small structural details of the image, like textures or thin features during the process, are common issues in abstraction.

Details such as textures and thin features can suggest depth and bring believability and vigor to images, as they do to realistic paintings. We would like to recover and in some cases exaggerate details lost through abstraction. Animal fur or whiskers, grass texture, bumps on rocks, and facial wrinkles are examples of details at risk of being lost through abstraction. Figure 1.5 shows a filtered 
image of the lion that has lost the details on the whiskers, fur, and the eye pupil. Although removing detail is the essence of abstraction, doing so can reduce the aesthetics of the result.
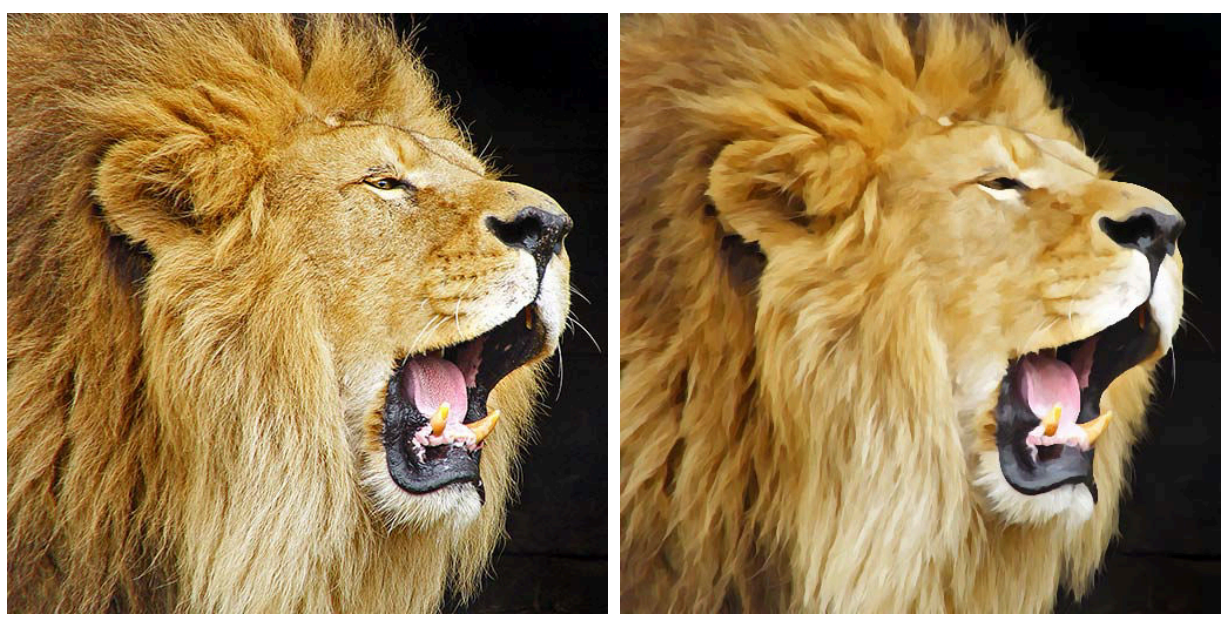

Figure 1.5: The filtered image of the lion [74] has lost details on the whiskers, fur, and the eye pupil. The original photograph shown on the left is courtesy of Tambako the Jaguar.

2. Simple color averaging produces dull colors. There is a problem with color and tone representation in abstraction. Averaging of the colors through an abstraction process would turn an image dull and uninteresting. We want to have the opportunity to recolor the abstraction. Merely averaging pixels generates dull colors. Varying the brightness or recoloring of abstraction or filtered image can introduce interesting effects. We aim to produce expressive abstractions by choosing more vivid colors. Although there is an existing body of work on recoloring, there is room for exploring this area; existing recoloring methods cannot cover specific styles such as Fauvism which needs more varied color assignments.

3. Uniformity appears in the shape and size of the elements in abstraction. Often, we have seen uniformity in shape and size of the textures in abstractions, which can be a natural outcome from many NPR abstractions. This effect appears both in filter-based and region-based methods. Abstractions by filter-based methods blur and flatten the jagged edges. Edges can assist in representing the texture and depending on the applications we may want to keep their shapes. 
Region-based abstractions often generate regular size regions which cannot capture small-scale structures and textures. Existing segmentation methods such as SLIC [3] generate regions of uniform shape and size. Other segmentation methods with more flexibility in region shape and size such as meanshift [25] or quickshift [131] also fail to represent very fine details like textures. We need an automatic algorithm that can represent regions with high irregularity in shape and size that can capture both main structures and thin features. To generate different art styles we would need a radical transformation of the elements. We need an abstraction framework that could be manipulated with some level of flexibility.

4. Conventional tone-preserving stippling struggles with extreme-tone regions.

Under conventional approaches, light regions become littered with stipples that are distracting and yet, because of their low density, cannot communicate any image features that may be present. At the same time, dark regions require immense quantities of stipples but because of small gaps between them, the effect is visually unpleasing. Additionally, low-contrast features in bright regions of an image will not be expressed with a tone-preserving stippling algorithm. There will simply not be enough stipples to indicate any contrast. For example, the cat's white whiskers against a bright background may no longer be visible after stippling. Although this is a natural outcome of tone-preserving stippling, we would still like to show these details.

By solving the above problems, we aim to express objects with more details and vivid colors to increase images' visual appeal. Like art movements that brought new ideas to the artists of the time, we want to generate abstractions representing fine features and wild colors to the abstracted image rather than smoothing the colors.

In summary, our research goals in this thesis focus on image stylization methods through manipulating the image regions and colors. We address the following objectives:

- Transforming naïve colorizations of abstractions into wild and colorful ones.

- Preserving fine-scale structures such as textures and thin features in abstractions, elements that are typically dropped under existing abstraction processes. 
- Generating abstractions that contain regions with highly irregular shapes and sizes, where conforming to the image content.

- Providing stylizations with radically transforming irregular regions into simplified structures.

- Preserving of both weak and strong structural elements in abstractions.

In the next section, we briefly propose our solutions to achieve these goals.

\subsection{Contributions and Thesis Structure}

This thesis provides a few abstraction methods to create stylized images that address the mentioned problems in NPR. It will demonstrate automatic systems to create and manipulate the stylized images that were generated through abstraction mechanisms: over-segmentation, colorization, stippling, and detail enhancement of abstractions. The thesis presents the following contributions:

- We introduced an abstraction method that locally emphasizes image details and mimics the artistic notion of indication [49]. We use the detail layer to reintroduce and exaggerate details in areas near strong edges. Introducing the details increases the aesthetics of the abstraction and enhances the local contrast. Moreover, we manipulate the colors and change the brightness across the filtered image while highlighting subjects in focus. The small shifts in colors creates more vivid abstractions than the original filtered image.

- We created a region-based abstraction that generates highly irregular oversegmented regions from a photograph. We create regions that can capture image elements of different shapes and sizes. Our approach can be seen as a hybrid of stroke-based and region-based stylization methods, generating complex region shapes that conform to local detail. We performed a preliminary investigation of stylizations that involve manipulation of the regions' colors and boundary shapes.

- We designed a recoloring framework for an over-segmented image, creating multiple recolorizations with just one palette. Our framework uses an ordering 
mechanism to prioritize regions' coloring to create wild and high contrast images. We also present various styles, softened recolored images by blending the colors, and simplified the recoloring by limiting the palette colors. Further, we generated new colorizations from a palette by applying different metrics and color spaces.

- We address two issues in stippling that appear in extreme-tone regions of the image. First, traditional stippling methods match the tone of dark areas by filling them with huge numbers of stipples; we instead cover each dark area with a polygon. Second, tone-based stippling can produce isolated stipples in light regions; we identify isolated stipples and remove them from the image. Moreover, we use a tone reversal mechanism for showing details in lighter regions. The overall contrast of the final result increases and shows clearer stippled images.

The remainder of this thesis is structured as follows:

2. Literature Review: The next chapter presents an overview of related work on region-based abstractions, NPR's abstraction methods, and detail enhancement methods. It covers recoloring approaches that were used mostly in NPR related literature.

3. Region-based Abstraction: This chapter provides an instance of regionbased abstraction method and its steps towards stylized abstraction. It explains a region growth mechanism that can abstract an image with highly irregular oversegmented regions. The chapter provides results from the original method and several examples from region boundary manipulations.

4. Recoloring of Region-based Abstraction: This chapter describes a recoloring framework for an over-segmented image. The recoloring system assigns wild colors from a palette to regions of the image to create vivid abstractions. It explains an algorithm constructed based on the widest path problem used for processing the regions. Moreover, it presents several stylizations obtained by manipulating the system variables.

5. Improving Stippling in Extreme-tone Regions: This chapter presents a method for improving stippled images in extreme-tone regions. It describes the method representing clusters of dark pixels by polygons rather than by a multitude of stipples. It also provides a solution for the intensity discontinuities resulting from thresholding using a Poisson blending operation. Further, it presents a postprocessing mechanism to eliminate spurious stipples that contribute little to image content. 
6. Hybrid Detail Enhancement and Abstraction: This chapter describes the hybrid detail and color enhancement for the stylization of photographs. The system represents relevant details in different image areas by employing the Poisson equation to score the details. Moreover, the chapter describes a simple method to brightening the abstraction. The net effect is a cheerful image in which some details are emphasized while others are suppressed.

7. Conclusions and Future Work: Lastly, this chapter concludes this thesis, evaluates our results, and gives directions on future work.

\subsection{Summary}

Some of the work in this thesis appeared previously in the following publications:

1. Rosa Azami and David Mould. "Image abstraction through overlapping region growth," In Proc. Graphics Interface (2020), pp. 66 - 73.

2. Rosa Azami, Lars Doyle, and David Mould. "Stipple Removal in Extreme-tone Regions," Expressive (2019), pp. 123-132.

3. Rosa Azami and David Mould. "Detail and Color Enhancement in Photo Stylization," In Proc. CAe (2017), pp. 1-11.

4. Rosa Azami and David Mould, "Artistic Recoloring of Image Oversegmentations," In Proc. Graphics Interface (2021). 


\section{Chapter 2}

\section{Literature Review}

This chapter gives an overview of existing research on image abstraction and stylization methods in NPR. The abstraction of photographs can be divided into regionbased, filter-based, stroke-based, and example-based methods. The chapter starts by reviewing past NPR methods in these categories, including a few approaches that applied boundary modifications. Then, it gives an overview of previous approaches to image detail enhancements. Next, it covers related work on recoloring methods and color palettes. The chapter ends with a discussion on the evaluation of NPR results and reviews a few fundamental methods that were used in our work.

\subsection{Abstraction Methods in NPR}

\subsubsection{Region-based Methods}

Several region-based algorithms have been introduced in non-photorealistic rendering. Regions are connected areas of the image, which are formed by groups of nearby pixels of similar colors or textures. A region's shape and size can differ based on the underlying algorithm.

Segmentation methods have previously been applied to images as a step on the way towards generating abstractions. DeCarlo and Santella [30] performed segmentation [5], then inferred the hierarchy of image structures from coarse to fine segments. Later, other researchers created a style rather than a simplified segmentation of the image. Mould introduced a stained glass filter [91] that mimics medieval stained glass windows. The regions were produced by an initial segmentation using meanshift [25] and then their boundaries were manipulated by morphological operators. Wen et 
al. [133] applied the meanshift segmentation [25] in an interactive manner to refine the segments. A user manually specifies which regions are background/foreground. Then, regions get colored. Xu and Kaplan introduced artistic thresholding [140] where a graph data structure was applied to the segmentation of a source image. They employed an energy function to measure the quality of different black and white coloring of the segments. Their results failed to show the high-level features that were crossing through the foreground objects. The problem was in the use of the $X O R$ to depict foreground objects on top of a background that varied between black and white.

Some region-based approaches simplify the image content into primitive geometric shapes. In Arty Shapes [125], the framework automatically chooses the shape (circles, triangles, rectangles, superellipses, and convex polygons) that best represents the region. More recently, the work by Faraj et al. [43] uses a topographical map to generate stylized images from simplified shapes organized in a tree structure. They use a dictionary to extract shapes and simplify the abstraction by removing small shapes based on a threshold value. However, similar to Arty Shapes, a limited number of geometric shapes are used and the method cannot represent various region shapes.

Graph-based optimization methods such as graph cuts [16,17] and GrabCut [112] also have been used as the underlying segmentation process for region-based stylization. Mould [96] proposed a detailed black and white stylization method, where graph cut was used in building and composing the detail and base layers. Zhao and Zhu [143] used GrabCut to segment the regions interactively in their abstraction system called Sisley.

Some NPR abstraction applied the SLIC (simple linear iterative clustering) method [2] to create regions. SLIC generates superpixels by clustering pixels based on their color similarity and closeness in the image plane. Doyle and Mould [38] used SLIC [2], to obtain tile boundaries of stained glass. Gerstner et al. [47] used SLIC to generate pixel art. SLIC is a proper choice of segmentation method for such applications that need regular regions. However, we need to capture details of different sizes and shapes and hence SLIC would not be a suitable method.

Watershed transformation and its hierarchical segmentation $[6,13,26]$ could be good candidates for abstraction. Arbelaez et al. [6] proposed a hierarchical segmentation which uses an oriented watershed transform to create initial regions from contours. The algorithm could be used for a coarse segmentation of the image. Because of the edge detector thresholding, lower gradient magnitudes will be halted, and thin 
and small features would be omitted. Recently, Cousty et al. [27] expanded the watershed hierarchical representation and proposed combinations of different hierarchical segmentation that can feature different aspects of the same image. Although watershed segmentation has not been used for abstraction purposes, this recent approach might be useful in the creation of a detailed abstraction. However, based on their results, while the algorithm can maintain some small features of the image, the segments are not capturing the full complexity of the object contours. In our work, for a highly detailed abstraction, we need an over-segmentation that will not eliminate small image features and that will conform well to the shape of the objects.

\section{Boundary Simplification}

Some NPR researchers, inspired by artists such as Delaunay, Kandinsky, Miró, and Matisse, created abstractions that transform complex geometric shapes into much simpler forms. Typical geometries included circles, squares or triangles, and other shapes resembling convex hulls. In Arty Shapes [125] the authors fit the segmented regions to shapes such as triangles or rectangles. Faraj et al. [43] proposed a hierarchical and geometrical image representation, called a topographic map, made of shapes organized in a tree structure. In addition to artistic abstraction, vectorization methods and image reconstructions could benefit from region simplification. In our design, the region's boundaries conform to the image structures and have the flexibility of an extreme transformation. Replacing the regions with shapes is not our goal.

Smoothing the boundaries can generate interesting effects. Fourier shape descriptors are an example method for boundary smoothness. Descriptors encode the shape of the regions by taking the Fourier transform of the boundary. Then, the original boundary can be recovered from the inverse Fourier transform. Doyle et al. [37] manipulated Fourier descriptors to smooth out the boundaries of the mosaics resulting in oval-like pebble shapes.

The previous approaches limit us to transformations close to the original shape of the region. The Ramer-Douglas-Peucker Algorithm [36], simplifies the region boundaries based on a piecewise linear curve which removes vertices based on a measure of error. This method would keep the shape of the structures very close to the original boundary; yet, it can retain a certain level of complexity even after simplification. A user can decide the level of simplification by adjusting a threshold for computing the 
error. We apply the RDP algorithm to simplify our region boundaries.

\subsubsection{Filter-based Methods}

Several filter-based methods in the literature produce abstract and artistic images. In the review of these filters, our attention goes to the flattening, edge preservation, and texture preservation of the results. We need an edge-aware filter, able to preserve both strong edges and weak edges depending on the surrounding context.

Papari et al. [104] introduced a painterly filter based on the Kuwahara filter [70]. They employed different weighting functions to preserve corners and edges. However, their method fails to capture directional features and results in clustering artifacts. Kyprianidis and Döllner [73] presented an edge-aware abstraction framework, based on the bilateral filter and used a smooth tensor field to capture local structures. Later, a generalization of the Kuwahara filter [74] and a multi-scale version of anisotropic Kuwahara filter [71] was introduced, which led to images with more painterly appearance. These filters have impressive abstraction results and preserve the local structure and directional image features, but they do not capture the textures or thin features. Moreover, because of using the directional field for capturing the local orientation, the smoothed image would have some deviation at weak edges. The distortion caused by deviation at weak edges makes it a poor candidate for our goal to retrieve the details.

Winnemöller et al. [138] presented automatic realtime video and image abstraction. By approximating images using anisotropic diffusion, they reduced contrast in low-contrast regions. However, they could not get rid of high-contrast texture lines without extreme abstraction of the low-contrast areas, while in our work some indication of image detail would suffice to capture the essence of textureness in the image. Orzan et al. [101] generated abstracted images by performing a Poisson reconstruction on extracted edges of the image. Their technique smooths weak edges and keeps the strong structural information. However, sometimes it over-simplifies shapes.

Median filters $[58,59,108]$ have been used for smoothing and especially noise reduction, but compared to bilateral filters $[39,40,130]$ they failed to preserve even strong edges. Kass and Solomon [65] introduced families of median filters, the smoothed local histogram filters that could preserve strong edges. These filters were based on derivatives and integrals of locally weighted histograms over large neighborhoods. One of these variations is called the closest-mode filter which can preserve strong 
edges. They used the detail layer for sharpening the effect; however, the result had extreme artifacts. They had to use a diffusion operator to reduce artifacts.

The geodesic filter [28] and its variants such as the cumulative range geodesic filter (CRGF) [94] try to smooth the image while keeping edges sharp. The textured regions will be abstracted through CRGF, while irregular shapes and ragged edges will be preserved. Since we are interested in capturing the irregularity of the textures, the CRGF filter is a good basis for our work.

Semmo et al. [118] proposed an stylized abstraction which transforms images into an oil paint look. They quantize the input image by using color palettes. To mimic the paint texture on canvas, they extract local orientations by means of smoothed structure tensor and line integral convolution [19]. The focus in this work was mostly on capturing the paint texture rather than the image texture. They used a flow field to generate the brush textures, so small structures and thin features can not be present in their results.

\subsubsection{Stroke-based Methods}

Several NPR researchers created abstractions using strokes, such as short lines, circles, and brush textures. In stroke-based methods, a common methodology is iteratively placing brush strokes with different color, size and orientation. Various artistic styles could be simulated based on this method. Haeberli [50] placed brush strokes in a stochastic distribution in the neighborhood of a manually driven cursor. Hertzmann [53] used a random order to render the strokes to prevent an undesirable appearance of regularity in the brush strokes. Zheng et al. [144] applied strokes in the context of 3D painting and animation where paint strokes change shape and length by movements of the camera. Their results benefit from modeling continuous and long brush strokes with variable size and shape. Other techniques, such as line drawings $[63,84,124,139]$, pen-and-ink illustrations [34,136, 137], and hatching [56,62,142] use strokes to abstract the image with highlighting the feature lines.

Stippling is another illustrative technique, which uses small dots rather than line segments to abstract the image. Most early stippling methods in computer graphics focused on achieving an even distribution of points, free of visual artifacts. One method of achieving this outcome is by means of Lloyd's algorithm [83]. Later, other approaches $[32,57,116]$ improved this method to converge faster and preserve image structure. Secord [116] proposed weighted Voronoi stippling, able to preserve some 
image structures through tone matching. Deussen et al. [31] proposed a method based on the Linde-Buzo-Gray (LBG) algorithm. It represents details better than Secord's algorithm while converging faster.

Example-based stipplings $[67,88]$ have focused on hand-drawn stipple art, with attention to aspects such as stipple distributions, dot shape, and grey-scale characteristics. Other higher-level artistic processes are considered as well [87], but current NPR algorithms are not able to automate them. Kim et al. [67] model stipple distributions from individual artists, then synthesize new distributions with similar statistical properties using tone maps to guide the stipple density. Martin et al. [88] discussed the grey-scale properties of hand-drawn stipples.

Structure-aware image reproduction [23,103] shifts the emphasis away from tone reproduction towards detail and texture representation. Mould [92] proposed a weighted-graph algorithm that favours stipple placement at high-gradient locations. Using a shortest-path algorithm, the image plane is explored while placing stipples at the frontier whenever a distance threshold is exceeded. With a similar objective, Schlechtweg et al. [115] introduced stipplebots which emphasize image structure. Li and Mould [78] drew on their previous work on error-diffusion based halftoning [77] to emphasize contrast and structure in the stippled result. Pixels are processed in a priority order that emphasizes extreme dark or light pixels. When a stipple is placed at a location, the resulting error, with respect to tone, is distributed among neighbouring locations. By varying the distribution pattern, different stylistic effects are achieved.

Although there are many improvements in structure-aware stippling, previous methods could not handle the problems that appear in extreme-tone regions. Stippling methods require enormous numbers of stipples to represent the black areas of an image. In light areas, where there is no information of the image content, placing a few stipples will distract rather than inform the viewer. Moreover, showing details is difficult in light areas, since tone preservation enforces a low stipple count in lighter regions. In our thesis we offer solutions for these problems.

\subsubsection{Example-based Methods}

The goal of example-based rendering is to find a mapping between an exemplar pair. Hertzmann et al. proposed image analogies [55], which generalizes texture synthesis for the case of two corresponding image pairs. The method has been applied to 
applications including texture transfer, painterly rendering and super-resolution.

Example-based renderings have been adopted in hatching techniques $[12,61,62]$ and stippling styles $[67,87,88,126]$. Although example-based methods may handle some stylization concerns, the mathematical model used in example-based methods cannot account for all quality factors. For example, preserving the structures is ineffective in stylized images produced by example-based approaches. While examplebased renderings are usually effective for a wide range of artistic styles, the algorithm will not work in practice if training data pairs are inaccessible.

In recent years, learning-based methods have become popular among NPR researchers. Neural Style Transfer (NST) is a method of using neural networks to render a content image in different styles. Gatys et al. [46] employ a Convolutional Neural Network $(\mathrm{CNN})$ to create creative images by recombining visual content and style. Following the original work [46], various NST algorithms were proposed $[24,79,80,135]$ to transfer a style to images. These recent approaches tried to preserve the detail of the original and eliminate distortion and other artifacts in the output.

\section{$2.2 \quad$ Detail Enhancement Methods}

Texture and detail preservation in abstraction is one of our missions in this research. Here we refer to a few approaches to detail preservation that have appeared for different purposes.

Fattal et al. [44] proposed a method for rendering high dynamic range images on conventional displays. They manipulated the gradient field of the luminance image by attenuating the magnitudes of large gradients and then construct a low dynamic range image by solving a Poisson equation on the modified gradient field. The attenuation must penalize the larger gradients more heavily than smaller ones, thus could compress drastic luminance changes, while preserving fine details. Bae et al. [11] presented an approach to manipulate digital photographs using a combination of non-linear edge-preserving decomposition and linear analysis. They suggested different detail management applications, such as detail preservation and texture transfer from a model to a source image.

Paris et al. [105] introduced Local Laplacian Filters, which perform a local pointwise manipulation of Laplacian pyramids. Highly detailed images are generated by manipulating the coefficients of the Laplacian Pyramid around each pixel. 
While previous methods dealt with high dynamic range images, Boyadzhiev et al. [15] considered material perception in their work. They split the image into highand low-frequency subbands and manipulate them. They achieved different categories of effects through band-sifting operators such as shiny/glossy/metallic, bright/glowing and old/used/worn. High-spatial frequencies capture features (like small-scale bumps and wrinkles), and low-spatial frequencies mostly represent large-scale shading variations. This is the core idea in most detail enhancement approaches.

In our work, we extract the detail layer. The details retrieved from subtracting an edge-aware filter [94] present good connectivity. We post-process the details to better capture features before augmenting them to the image. We use the sticks filter [29], which was originally designed to reduce speckle noise and preserve linear structures. By applying the sticks filter on the detail layer we preserve and extend the thin features.

\subsection{Color Palettes and Recoloring Methods}

In this thesis, we present a non-interactive recoloring method, that uses a color palette for recoloring of the region-based abstraction. Here, we review the existing research on recoloring methods in NPR and color palette selection.

\subsubsection{Example-based Recoloring}

Recoloring methods were first proposed by Reinhard et al. [111], where the colors of one image were transferred to another. They converted the RGB signals to Ruderman et al.'s [113] perception-based color space $l \alpha \beta$. To produce colors they shifted and scaled the $l \alpha \beta$ space using simple statistics.

Neumann and Neumann [98] extracted palette colors from an arbitrary target image and applied 3D histogram matching. They attempt to keep the original hues after style transformation. They performed matching transformations on 3D cumulative distribution functions belonging to the original and the style images. However, due to the lack of spatial information, some problems appeared by unpredictable noise and gradient effects. They suggest using image segmentation and 3-dimensional smoothing of the color histogram to improve the result.

Levin et al. [76] introduced an interactive colorization method for black and white 
images. They used a quadratic cost function derived from the color differences between a pixel and its weighted average neighborhood colors. The user indicates the desired color by scribbling in the interior of the region. The colors automatically propagate to the remaining pixels. However, sometimes it fails at strong edges, due to a sensitive scale parameter. Inspired by Levin et al. [76], Yatziv and Sapiro [141] proposed a method based on luminance-weighted chrominance blending and fast intrinsic geodesic distance computations. Sykora et al. [128] developed a tool called LazyBrush for the colorization of cartoons which integrates textures to the images to create 3D-like effects [127]. Casaca et al. [21] used Laplacian coordinates for image division and used a color theme for fast colorization. Fang et al. [42] proposed an interactive optimization method for colorization of the hand-drawn grayscale images. To maintain a smooth color transitions and control the color overflow in the texture areas, they used a smooth feature map to adjust the feature vectors.

A few methods composite the colors for recoloring. The compositing could be achieved by using alpha blending [89] or the Kubelka-Munk [68] equation (KM). For a paint-like effect, NPR researchers mostly worked with the physics-based KubelkaMunk equation to predict the reflectance of a layer of pigment. Some researchers tried to mimic the actual artist decision of mixing the colors to generate the palettes. To improve the prediction of compositing colors, a data-driven color compositing framework by $\mathrm{Lu}$ et al. [85] derives three models based on optimized alpha blending, interpolation using radial basis functions (RBF), and KM optimization. Later, the KM pigment-based model was used for recoloring of styles such as watercolor painting [4]. The compositing by the KM model leaves traces of overlapping stroke layers which can produce nearly natural painting effects.

\subsubsection{Palette-based Recoloring}

The interest in colorization and recoloring methods opened up new research ideas on color palettes, rather than simple approaches like averaging colors [48]. A frequent interaction scheme has a user scribble each region of the image with a color, and the algorithm would do the rest of the work. Early methods to select the color palettes used Gaussian mixture model (GMM) [121] or k-means [22] to cluster the image pixels. Chang et al. [22] introduced a photo-recoloring method by user-modified palettes. To select the color palettes, they compute the k-means clustering on image colors, then discard the black entry. Tan et al. [129] proposed a technique to decompose an image 
into layers to extract the palette colors. Each layer of decomposition represents a coat of paint of a single color applied with varying opacity throughout the image. To determine a color palette capable of reproducing the image, they analyzed the image in RGB-space geometrically in a simplified convex hull.

Recent methods on generating the palettes have more flexibility for editing. Playful Palette [123] is a set of blobs of color that blend together to create gradients and gamuts. DiVerdi et al. [35] also proposed an approximation of image colors based on the Playful Palette. An objective function minimizes the distance between the original image and the recolored one, based on the self organizing map. While the method is faster, the quality is lower.

A few methods assign colors to regions. Qu et al. [110] proposed a colorization technique for the black and white manga using the Gabor wavelet filter. A user scribbles on the drawing to connect the regions; the algorithm then assigns colors to different hatching patterns, halftoning, and screening. Xu and Kaplan introduced artistic thresholding [140] where a graph data structure is applied on the segmentation of a source image. They employed an energy function to measure the quality of different black and white coloring of the segments. However, they failed to show the high-level features that were crossing through the foreground objects. There are a few approaches in the pattern colorization problem, intended to colorize graphic patterns [14,81]. Lin et al. [81] proposed a palette-based recoloring method. They learn and predict the distribution of properties such as saturation, lightness, and contrast for individual regions and neighboring regions. Pattern colorings are scored using the predicted distributions and color compatibility model by O'Donovan et al. [99]. Bohra and Gandhi [14] approached the colorization problem as an optimal graph matching problem over color groups in the reference and a grayscale template image. Similar to Playful Palette [35], the colorArt method of Bohra and Gandhi [14] enforces an equal number of colors in the reference palette and the template image, and cannot find matches otherwise.

\subsection{Evaluation}

Presenting research results requires convincing the audience about the value of the work. When developing algorithms, we want to show that the algorithms are useful; in NPR, when we create a style or artistic effect, we need to convince the reader 
that our results are interesting or expressive. Defining artistic goals in a useful and measurable way is much more difficult. Yet, we need to evaluate our result in some way.

One possible way is user studies. However, Hertzmann [54] argued that poorlydesigned studies can damage the creativity and exploratory aspects of NPR research. It can mislead the readers if the study is not well designed. Each study is limited by specific measurements and assumptions of the design, so the statistics from a study may not justify the method. Hertzmann claimed that the NPR community does not have a good sense for how to make effective use of studies.

Hall and Lehmann [51] suggested avoiding measurement in favor of appreciation. They argued that NPR should borrow evaluation techniques from art history. Hall and Lehmann pointed out that NPR researchers sometimes cannot characterize the objectives of the style properly, while training in art history would make evaluation easier. However, Mould [95] disagreed on this vision. In reality, not all the practitioners would have the opportunity to learn art history and it is not the only area of knowledge that satisfies a proper evaluation of NPR work. He reminded us that in NPR research, structural approaches have been used for evaluating the images, and lack of knowledge on the art concepts should not be a barrier in evaluating an NPR work.

Direct objective measurement of properties of the images is preferred but is hard to apply, because it requires a customized metric function estimating the quantity of interest and it is unable to provide definitive answers directly. Usually, for evaluating image processing applications, availability of the ground truth makes it possible to apply a direct objective measurement. However, we normally do not have ground truth from the stylized images to measure the objectives.

Mould [95] described a structured approach for subjective evaluation. He suggested to be specific in initially describing objectives, and then to return to the same list of objectives in the evaluation. To evaluate our abstractions, we are following the design that Mould suggested. We need to break down the target style into components that can be separately evaluated. Knowing the characteristics of the style would help in designing the algorithm. We need to make sure that the most important elements be identified through the evaluation.

A clear problem statement is important in deciding what form evaluation should take. For example, in our abstractions, we care to retain the important details. Later 
in evaluation, the output images should be able to support this characteristic of our interest in the generated style. In general, in our evaluations, we target the important components of our abstraction framework such as preserving the details and capturing structures of different scales and shapes, enhancing the contrasts and flexibility for reshaping and recoloring.

Moreover, illustrating several example images can show different aspects of the method. We will present our abstractions with images of different compositions and use different parameter settings. It will help the reader to better understand the objectives of the method. Finally, it is important to have a good discussion of the results and draw attention to details in the examples that states the method's success. In our work, we have a few side-by-side comparisons of previous approaches and point out the specific differences between images produced by different algorithms.

\subsection{Fundamental Material}

In this section, we review some known methods that we have employed in our work.

Poisson Equation: The Poisson equation has been used effectively as a guided interpolation approach for image synthesis and processing [41,101,107]. In general, a Poisson equation with Dirichlet boundary conditions is defined as [107]:

$$
\Delta f=\operatorname{div} \mathbf{v} \quad \text { over } \quad \Omega \text {, with }\left.\quad f\right|_{\delta \Omega}=\left.f^{*}\right|_{\delta \Omega}
$$

where $\operatorname{div} \mathbf{v}=\frac{\delta u}{\delta x}+\frac{\delta v}{\delta y}$ is the divergence of $\mathbf{v}=(u, v)$ and $f^{*}$ is a scalar function defining the boundary conditions.

Elder and Goldberg [41] introduced an image editing system in the contour domain. Their system reconstructs the image intensity from brightness at edges. They assumed that in regions other than edges, the intensity function approximately satisfies the Laplace equation $\Delta I(x, y)=0$, and therefore solving the Laplace equation provides a good reconstruction of the original intensity. Perez et al. [107] also used the Poisson equation as an interpolation mechanism. Their proposed method smoothly imports source image regions into a destination image and can affect the texture, illumination, and color of objects within the selected region. Orzan et al. [101] used a Poisson reconstruction method for image stylization, controlling local detail levels in an input photograph. Orzan et al. [102] and Jeschke [60] used the Poisson equation to interpolate colors smoothly on either side of a boundary curve. The Diffusion Curve 
Image (DCI) introduced by Orzan et al. [102] specifies colors on either side of Bezier curves. Jeschke [60] improved DCIs by introducing new boundary conditions midway between the diffusion curves.

Like previous methods, we solve the Poisson equation, using it for both color manipulation and detail enhancement. We obtain a smooth map over the image considering two main boundary conditions: edges of the image and the ridges of the distance transform map obtained from edges. Jeschke [60] also used the term "ridges" to refer to curves midway between the diffusion curves.

Connected Component Analysis [119,120]: Connected pixels are any set of pixels with similar intensities that are joined within a boundary. Any maximal region that contains the connected pixels is called a connected component; the connected component partition segments the image. Connected components analysis has been applied for many image processing applications such as object extraction and line detection. In this thesis, we have used connected component analysis in grouping the pixels in the detail layer [11].

Edge Detector: Another widely used method in image processing and computer vision is edge detection. The Canny edge detector [20] has been commonly used in determining the locations where there is a high alteration in the contrast in an image. Such locations often form the boundary of objects in a scene.

Widest Path Problem: Pollack [109] introduced the widest path or maximum capacity path problem which appeared in network routing and transportation problems. The widest path algorithm finds a path between two designated nodes in a weighted graph that maximizes the weight of the minimum-weight edge in the path. We use this algorithm to determine the regions' order of processing by constructing a tree on a graph of connected regions.

Detail Layer Decomposition: Durand and Dorsey [39] applied a contrast reduction by decomposing an input intensity into two scales. They used the bilateral filter [130] to produce a large-scale layer called base, and a detail layer which is the difference between the input intensity and the base layer. Similar to Durand and Dorsey [2002], Bae et al. [11] defined the base layer and detail layer from the input image for tone management of photographs.

Inspired by the previous approaches, in this thesis, we decompose an image I into base B and detail D layers:

$$
B=C R G F(I) \quad, \quad D=I-B
$$


where CRGF is an edge-aware smoothing filter called cumulative range geodesic filter [94]. The detail layer which is also called the residual layer, gives information about the distribution of the details in the image. In the areas with textures or small-scale features, the detail layer shows higher density in the distribution of details while in the flat areas, the detail layer is sparse or empty. 


\section{Chapter 3}

\section{Region-based Abstraction}

\subsection{Introduction}

Image stylization has seen a tension between abstraction, which removes detail from input images, and media emulation, which often adds detail such as watercolor texture or brushstroke direction. However, while the resulting images can contain fine-scale details such as paint texture, such details are often dissociated from any fine-scale detail that had been present in the input. The resulting images often show uniformity in shape and size of the abstraction primitives. Fine textures and small structures can characterize the objects in the image and presenting these features can increase the visual appeal by suggesting depth to an abstraction work. Abstraction methods often fail to capture the small structural components. This issue appears both in filter-based and region-based methods. Abstractions by filter-based methods may blur edges. Region-based abstractions often generate uniform-size regions which cannot capture small-scale structures and fine textures.

This chapter presents an automatic oversegmentation algorithm that can create regions with highly irregular shapes and sizes; the resulting regions can then be used as stylization primitives $[125,140]$. Sample results from our approach appear in Figure 3.1.

This approach uses region-based abstraction to modify an input photograph, where the image plane is covered by overlapping irregularly shaped regions that approximate the image content. Unlike traditional segmentation methods, or superpixel methods such as SLIC [3], we deliberately create non-compact regions, with the 

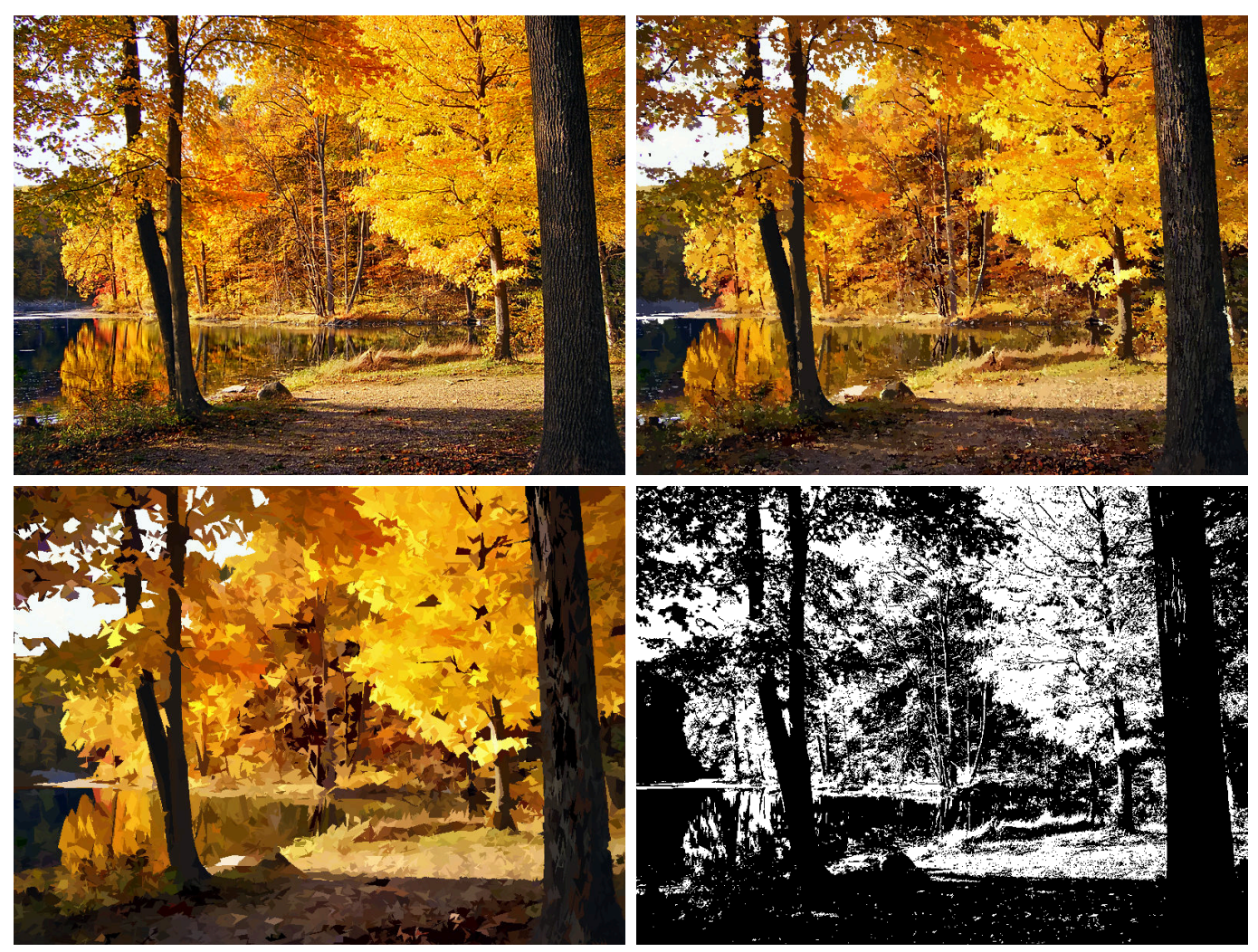

Figure 3.1: Abstraction result. Left to right: input image, modal region colors, simplified boundaries, black and white.

jaggedness and complexity of region shapes creating significant visual interest. Figure 3.2 shows some sample region shapes generated with our method. Image detail is preserved because the region boundaries conform to image edges, while variation in final region size allows very small details to be kept. We do not add any detail based on simulated media, though that could certainly be added if desired. Our regions have the following properties:

- Irregularity: generated regions have high irregularity in shape and size.

- Detail preserving: capture the small details of the image, like high-frequency textures or thin features.

- Structure preserving: can conform to edges within the image, which enables capturing the structures. 

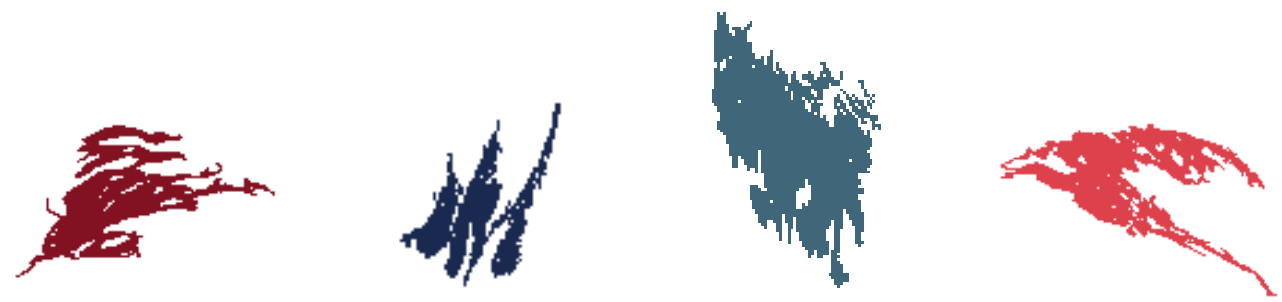

Figure 3.2: Irregular regions generated by our method.

The process is entirely automatic. From the initial image, we compute a detail map by subtracting a smoothed version [11]. Connected components of a thresholded detail map generate seeds for a region growth process. Seeds are ordered based on the residual values, and seeds with smaller residual values are expanded first. When all regions have been found, we assign a color to the visible portion of that region.

This chapter makes the following contributions:

- Overall, we propose a mechanism for abstracting images through highly irregular region shapes that nonetheless conform to image content. Our approach can be seen as a hybrid of stroke-based and region-based stylization methods.

- We introduce a region-growing algorithm that generates intricate region shapes that conform to local detail. Unlike traditional oversegmentation methods, we emphasize the irregularity of region shape even in uniform areas.

\subsection{Proposed Method}

Our method is a region growth approach guided by local colors, where regions are the key elements of the abstraction. Given an input image $I$, our goal is to generate a set of regions $\left\{R_{i}\right\}$ that capture the image's important structures. For example, image textures are conveyed by the irregular shape of the regions. Our method has three main steps:

- Seed placement: we identify a starting point for each region; this is done by finding connected components from a thresholded detail layer. Each connected component above a minimum size generates one seed. 


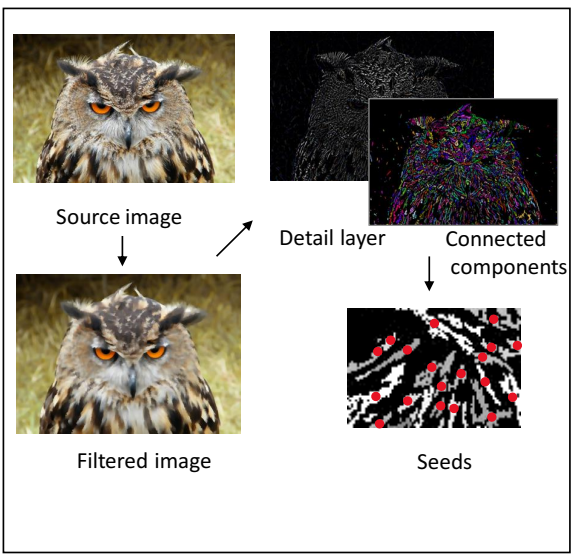

Seed placement

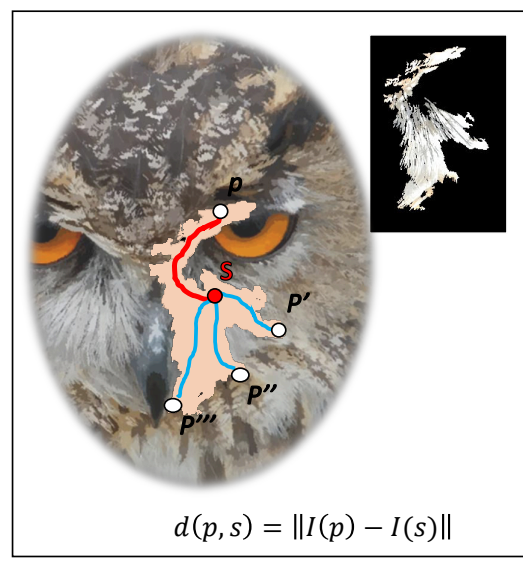

Region generation

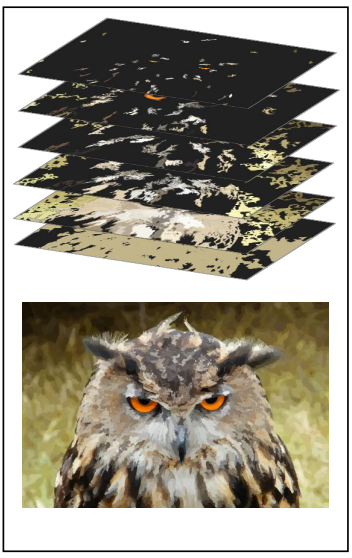

Rendering

Figure 3.3: Region-based abstraction stages. We compute a detail layer from input image and its filtered one. Then, we generate initial seeds for each region from the connected components of this detail map. For each seed, we expand a region. When all regions have been found, we color the output by assigning to each region the mode of the pixel values in the visible portion of that region.

- Growth mechanism: a region grows until a stopping criterion is reached. We halt expansion locally when the incremental cost becomes too large; when expansion is not possible in any direction, the region is finished.

- Rendering: we place regions in order of size: on the bottom, the largest regions cover the background, and smaller ones come on top providing detail. The final color of each region is the modal color of its visible pixels.

Figure 3.3 provides a visual depiction of these stages. In the following, we describe each step of the algorithm in more detail.

\subsubsection{Seed Placement}

We want to preserve the visual features of the image. "Visual feature" is a broad category encompassing all manner of small-scale elements that can help a viewer recognize objects and interpret the image, and includes disparate entities including small structures such as distant objects, material details such as cracks or bumps, lighting phenomena such as highlights or shadows, and more. Each seed will later form a region; a desirable seed distribution places one seed on each visual feature. Ideally, we want to avoid putting extra seeds within a visual feature as doing so could 
create many nested regions, which are not visually appealing.

We use image residuals to guide us in placing the initial seeds. We use the heuristic that extrema in the residuals are likely to correspond to features of interest. One strategy might be to use non-maximal suppression to identify extremal points. However, for noisy or complex images, the number of extrema may be excessive. We approximate the visual features by first computing a three-way threshold, dividing the image into areas of low residual, large positive residual, and large negative residual. We then compute the connected components of the resulting field.

Note that this process guarantees separation of locally bright from locally dark details, providing a seed (and subsequent region) for each; were one to threshold on the absolute value of the residuals instead, dark and light extrema would be confused and useful detail would be lost.

Figure 3.4 provides some intuition. On the left, it shows the absolute values of the residuals. On the right, it shows both positive and negative residuals, where darker pixels have negative signs, and lighter pixels have positive signs. Residual of value zero corresponds to a gray value of 128 . The owl's feathers are far more distinct when the sign of the residual is retained.

To produce the detail layer (residual layer) [11], we smooth the input image $I$ using the cumulative range geodesic filter (CRGF) [93] and subtract it from the original:

$$
B=C R G F(I) \quad, \quad D=I-B
$$

where $\mathrm{B}$ is the base and $\mathrm{D}$ is the detail layer. The spatial variations in the detail layer indicate where the image's visual features are located. Featureless areas, such as flat surfaces, often have zero or extremely small residuals, whereas areas with varied residuals contain visual features.

We find all connected components with positive and negative labels, discarding components that are too small (less than 10 pixels in size). We compute one region per connected component, starting the region growth at the component's median pixel position. Figure 3.5 illustrates selected seeds - red points - on the connected components of the negative detail layer.

Figure 3.6, left to right, shows connected components of the thresholded positive and negative residuals in color coded segments. The third and fourth images are 

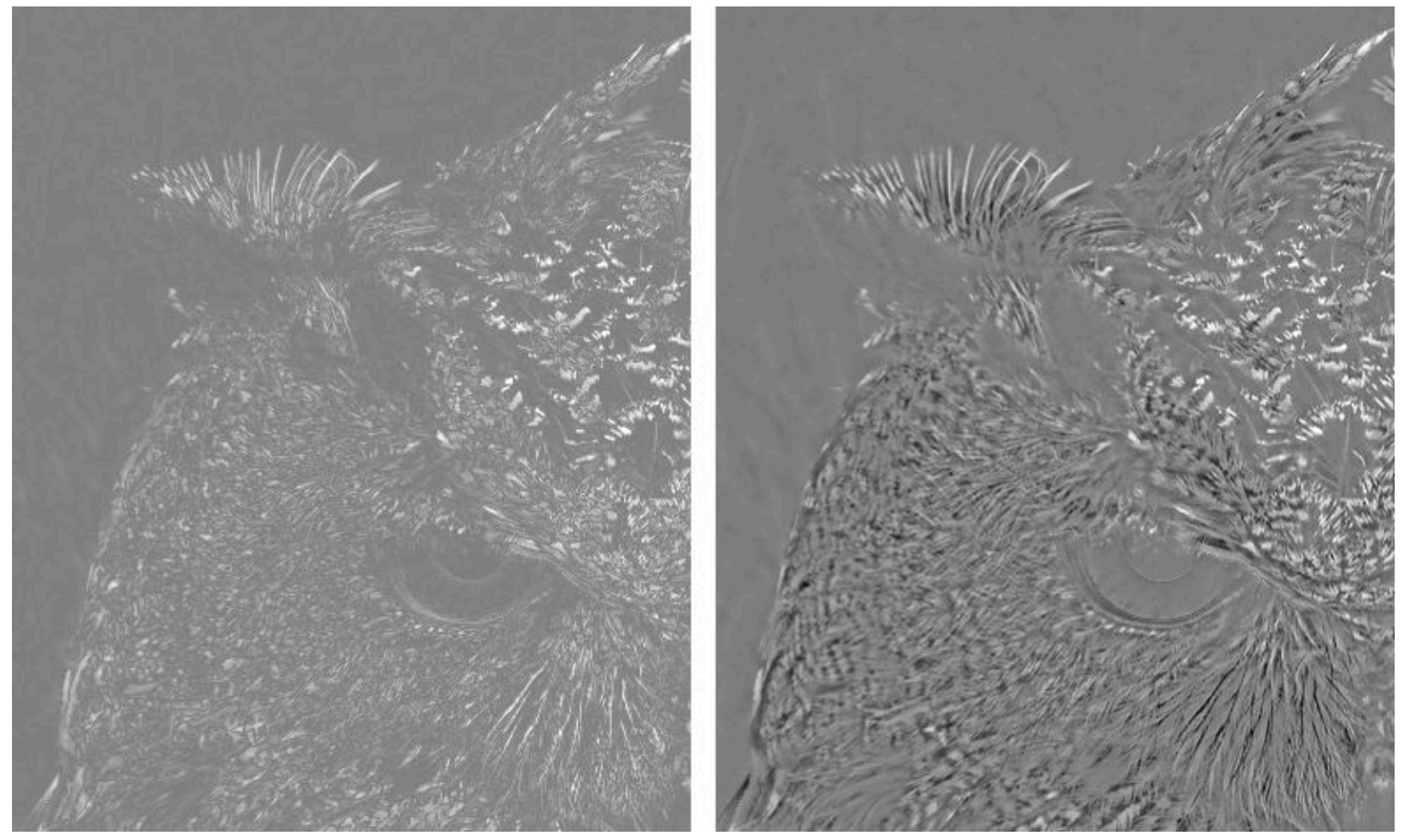

Figure 3.4: Left to right: absolute residuals vs positive/negative residuals.
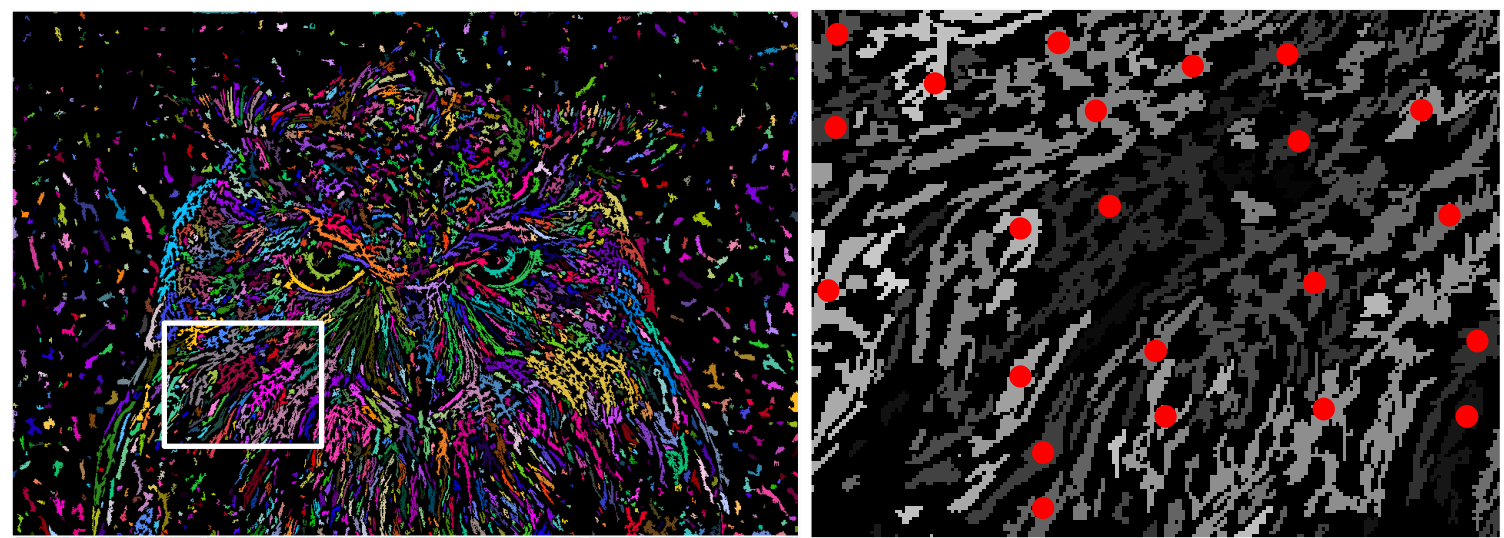

Figure 3.5: Seed placement. Left: all connected components of the (negative) detail layer in owl image. Right: close-up of the rectangle area on the left image, showing the selected seeds for each connected components of the detail layer. 
our results from over-segmentation and recoloring of regions. To capture the visual features, connected components of residual maps offered a good approximation of the seed distribution. Many generated regions are closely related to the corresponding connected components.
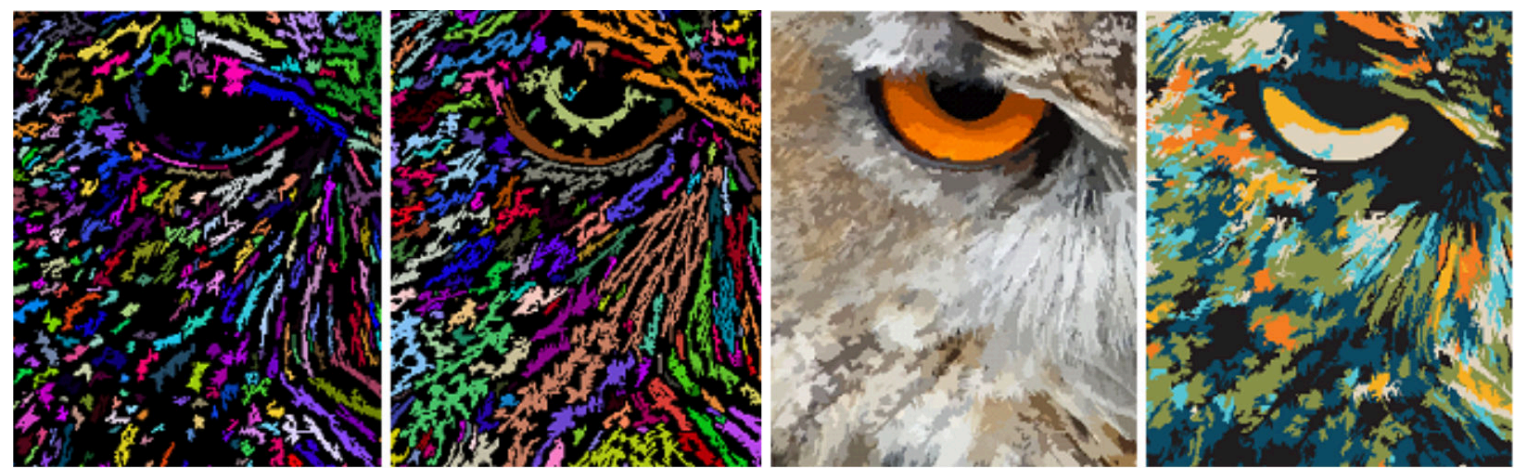

Figure 3.6: Residuals divided into positive and negative connected components (color coded) have a close relation with the image content.

\subsubsection{Growth Mechanism}

The seeds are now treated individually, with a region grown around each one, following a best-first order in an 8-connected graph. We continue adding pixels to the region, ordered by increasing cumulative range geodesic distance [93], until growth in every direction has halted. Effectively, this process involves computing shortest paths where the edge weight to a node $p$ is the color distance to the seed; $D_{c}(p, s)=\|I(p)-I(s)\|$, where $I($.$) denotes image color or intensity at each pixel location, and \|$.$\| is the$ Euclidean norm. In our process, we used the squared color distance in Lab space; we found that duplicating the luminance factor and squaring the distance $D_{c}$ led to greater ability to distinguish fine detail.

Each seed is assigned a unique region ID, used to label each pixel in its region. A given pixel can belong to many regions simultaneously; the IDs of regions passing a pixel will be stored in the pixel's data structure. Once all the regions are generated, each pixel carries several region IDs, where the last one represents the final region ID. The overlapped regions leading to subdividing the larger regions. In the end, we assign each pixel the ID of its smallest region; a connected component analysis on the image pixels assigns a unique region ID to pixels belonging to a fragment region. 


\section{Region Growth Termination}

Our region growth process can be viewed as a single-source shortest path problem, where we compute range geodesic distances to a tree of nodes surrounding the seed. We expand the region by recursively adding the neighbours of each node, tracking the total path cost of each node from the seed. When the incremental cost along the growth path exceeds a per-region parameter $T$, we halt further growth along that path. When all growth paths have been halted, the region is considered complete. In our implementation, we do not explicitly terminate growth through a node; rather, the boundary nodes that we are considering adding are stored in a priority queue, and we implicitly halt progression by not adding potential successor nodes into the queue.

We decide whether or not to locally halt progression based on whether or not the cost of going in the current direction is considered to be large in the context of the current region. We operationalize "large" as follows. The first time a node added to a region is $h$ edges from the seed, we store its accumulated cost. This cost provides a baseline value, say $T$; we increase the baseline by a factor $k$, using $h=10$ and $k=100$ in our examples. Subsequently, for every pixel we consider adding to the region, we compare the total weight of its most recent $h$ edges, say $W_{h}$, with the baseline T.k. When $W_{h}>T . k$, we terminate progression. In other words, any increase beyond a factor of $k$ in the rolling-average path cost halts region growth in that direction. Figure 3.7 illustrates a sample region growth from seed $s$ and the terminated pixels on the boundary of the region. Algorithm 1 shows the steps.

\subsubsection{Rendering}

We want to construct an ID map where each pixel in the image has a sing;e region ID. We first sort the generated regions from larger to smaller and process them in sorted order. Processing a region means assigning to all its pixels the ID of the region. In the end, each pixel has the ID of the last (smallest) region it was inside. Once we have assigned preliminary region IDs to all pixels, we finalize the IDs by performing a connected component analysis of the ID map. Doing so gives us different labels for region fragments that have been separated by other regions. The process of going from overlapping regions to a single ID per pixel is called flattening. 

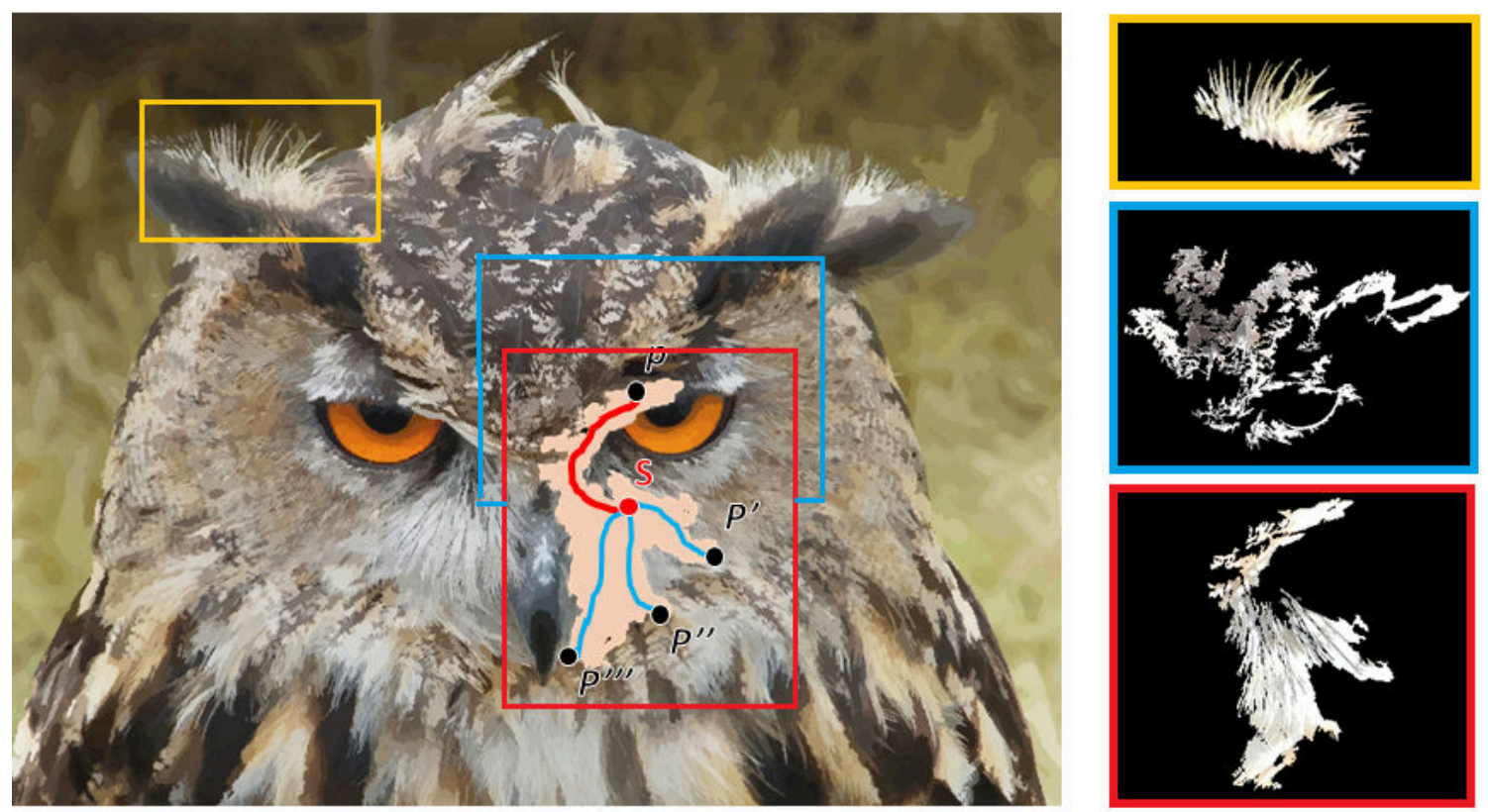

Figure 3.7: Examples of regions generated by the free-growth mechanism. The red square on the image illustrates several paths from seed to pixels terminating the region's growth.

Figure 3.8 illustrates the rendering process. From left to right, it shows the overlapping of the grown regions, the contours after flattening process and the rendered image. For each of the final flattened regions, we compute the average color and apply this color to its constituent pixels. The final result is an oversegmented abstraction of the image. Figure 3.9 shows region contours resulting from rendering the connected components. Note that our approach is able to convey textured areas of the image, such as the grass and tree trunk, by irregularly-shaped regions.
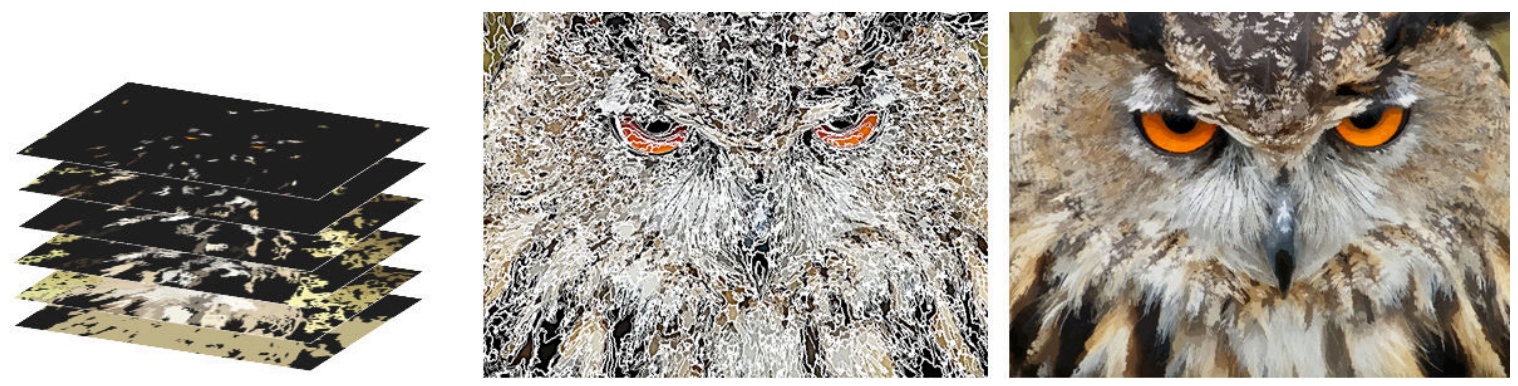

Figure 3.8: Flattening. Left to right: overlapped regions, the contours after flattening process and the rendered image. 

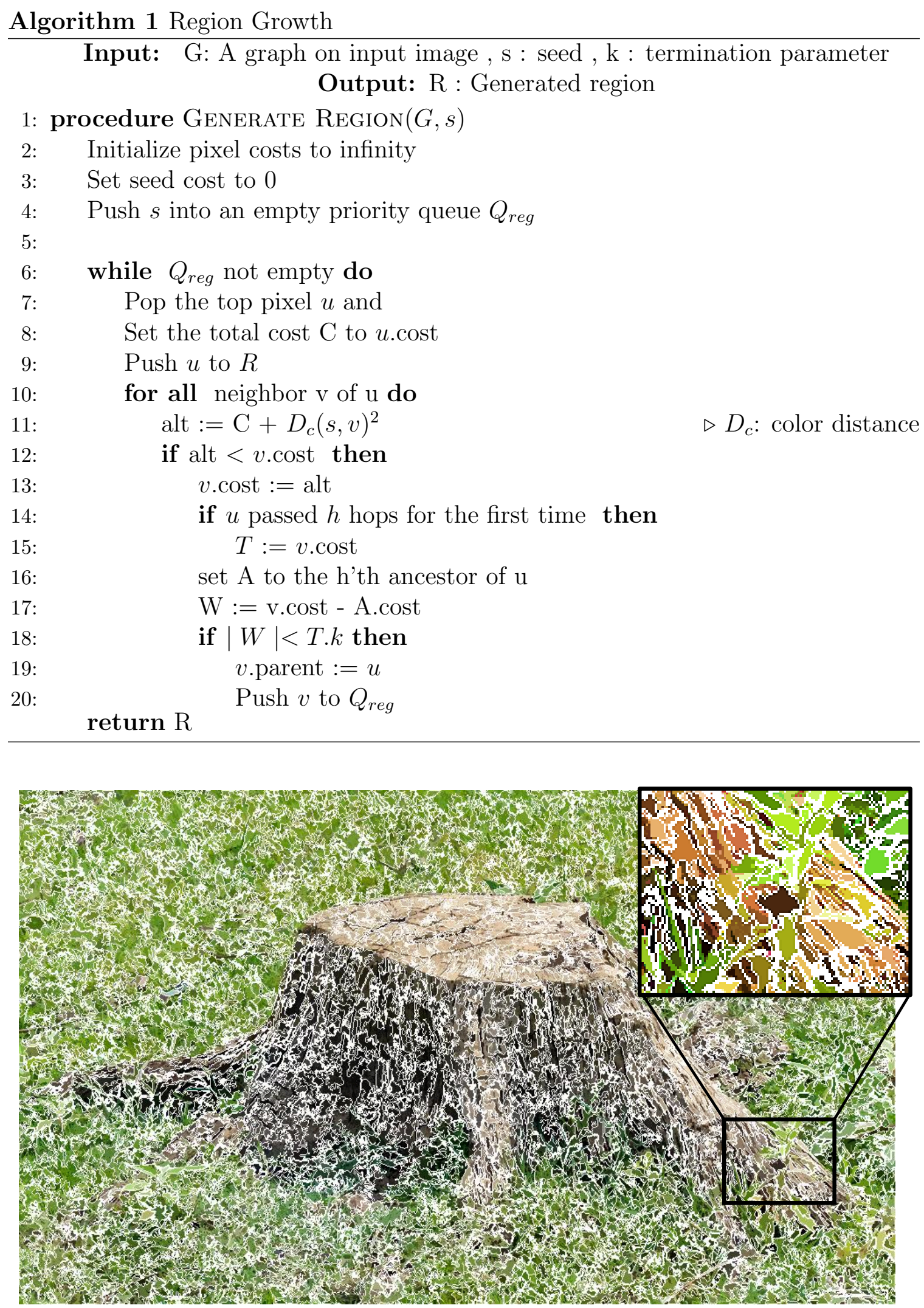

Figure 3.9: Region boundaries in stump image. 
Often, the parts of the image that have little detail do not generate any seeds, and consequently, these areas may not be assigned any region ID before the region growing process completes. The connected component analysis guarantees that all pixels will be given a region ID.

However, uniform areas of the image may be covered by large regions with little interior detail. For many applications, this may be desirable. For cases where it is not, we suggest covering uniform portions of the image by a template of textured regions from another image, with a mask selected manually. In Figure 3.10, we show an example of a textured image and the corresponding regions suitable for use as template regions.
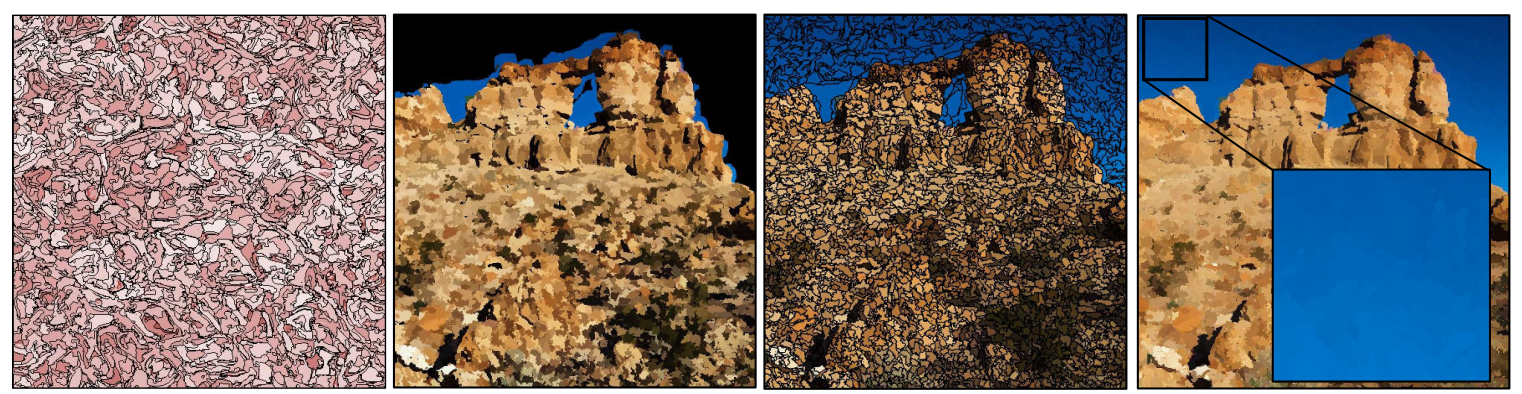

Figure 3.10: An image of a noisy texture used as background texture for the sky in the arch image.

\subsection{Reshaping Region Boundaries}

We can use the regions directly for image abstraction. In addition, we can manipulate the regions, for example altering region shapes or manipulating region colors. In this section, we undertake a preliminary discussion of the region boundary reshaping and in the next chapter, we introduce a method for recoloring the regions.

\section{Contour Simplification}

Drastic shape simplification has been used for stylization previously $[43,125]$, often using highly simplified shapes. We simplified the region boundaries based on the Ramer-Douglas-Peucker (RDP) Algorithm [36], which simplifies a piecewise linear curve by removing vertices based on a measure of error. This method would keep the 
shape of the structures very close to the original boundary; because we began with elaborate shapes, we retain a certain level of complexity even after simplification. A user can decide the level of simplification by adjusting the threshold $\epsilon$ for computing the error. In Figure 3.11, $\epsilon$ is set to 4 for both images. Higher thresholds create more drastic shape simplifications. We showed the simplified boundaries of initially generated regions and the flattened ones. Simplifying the flattened regions will create gaps between connected regions. We used a smoothed version of the original image to cover the background. Boundary simplification of the larger regions can produce interesting results, though the effect on small regions is negligible. We rendered the simplified boundaries of the original regions in all of our results.

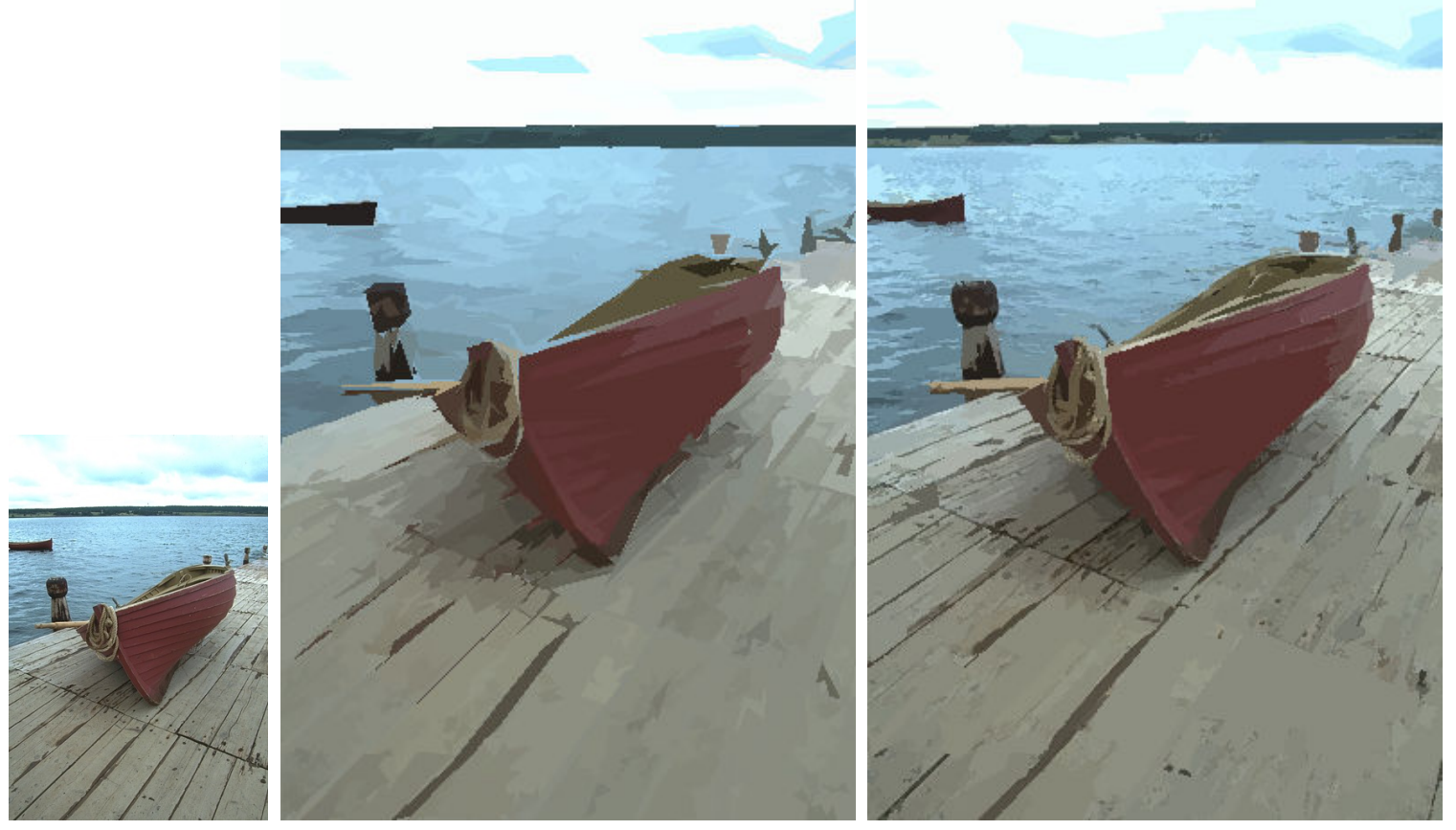

Figure 3.11: Abstractions after boundary simplification by Ramer-Douglas-Peucker Algorithm $(\epsilon=4)$, with initially generated regions (middle) and with flattened regions (right).

\section{Boundary Smoothing}

Alternatively, we suggest smoothing the region boundaries in frequency space, computing Fourier shape descriptors $[18,69]$ and then truncating the coefficient sequence. 
This process produces a high level of abstraction. Some results appear in Figure 3.12, where we retained the first 7 coefficients.
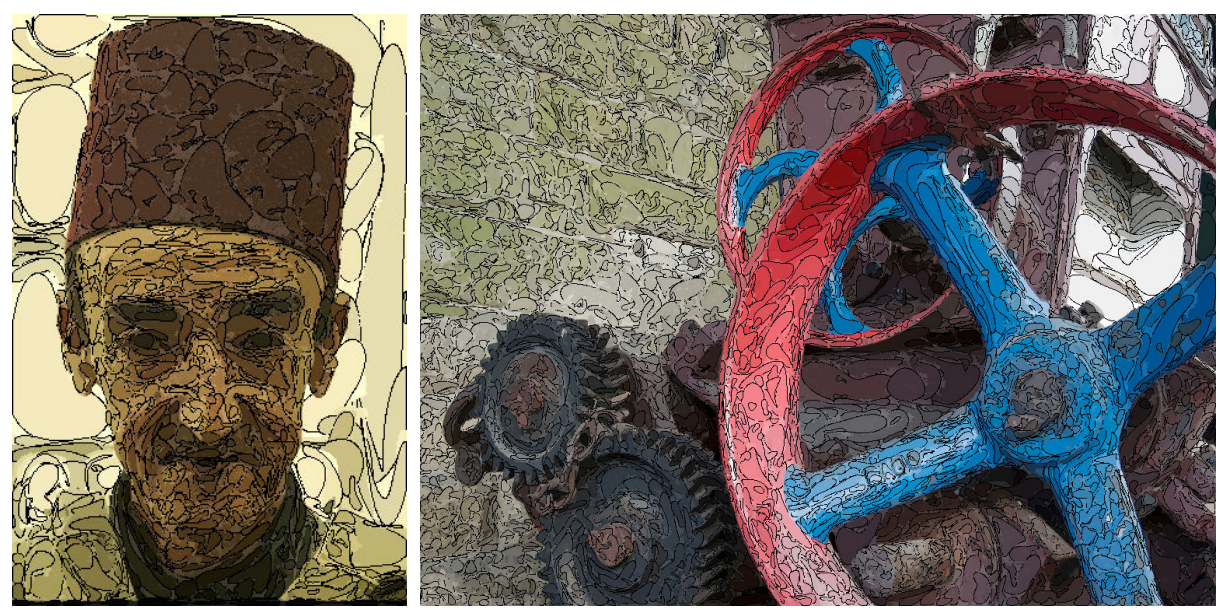

Figure 3.12: Regions simplified with boundary smoothing using Fourier shape descriptors.

\subsection{Results and Discussion}

We give more examples of our region-based abstraction in Figures 3.13 and 3.14. Original images of the examples presented in Figures 3.9, 3.10 and 3.12 can be seen in Figure 3.15. We encourage readers to zoom in to better observe the abstraction effects. We wanted thin features and textures to stay visible after abstraction. In the market image, the texture of the bale of hay has been captured, and boundaries of the wheat sheaves kept their jagged shapes. Some small objects on the back shelves and hanging objects are abstracted. Our abstraction preserved strong edges such as the bars of the booth. On the right, the simplified version of the abstraction by means of overlapped regions created more artistic results.

The temple image shows how our abstraction mechanism can keep both isotropic structures and anisotropic textures at the same time. The abstraction process retained irregular textures in the background. The bricks have low contrast textures which are not prominent but are still captured by the regions. Abstraction through boundary simplification deforms the regular structure of the bricks, which might be undesirable. 

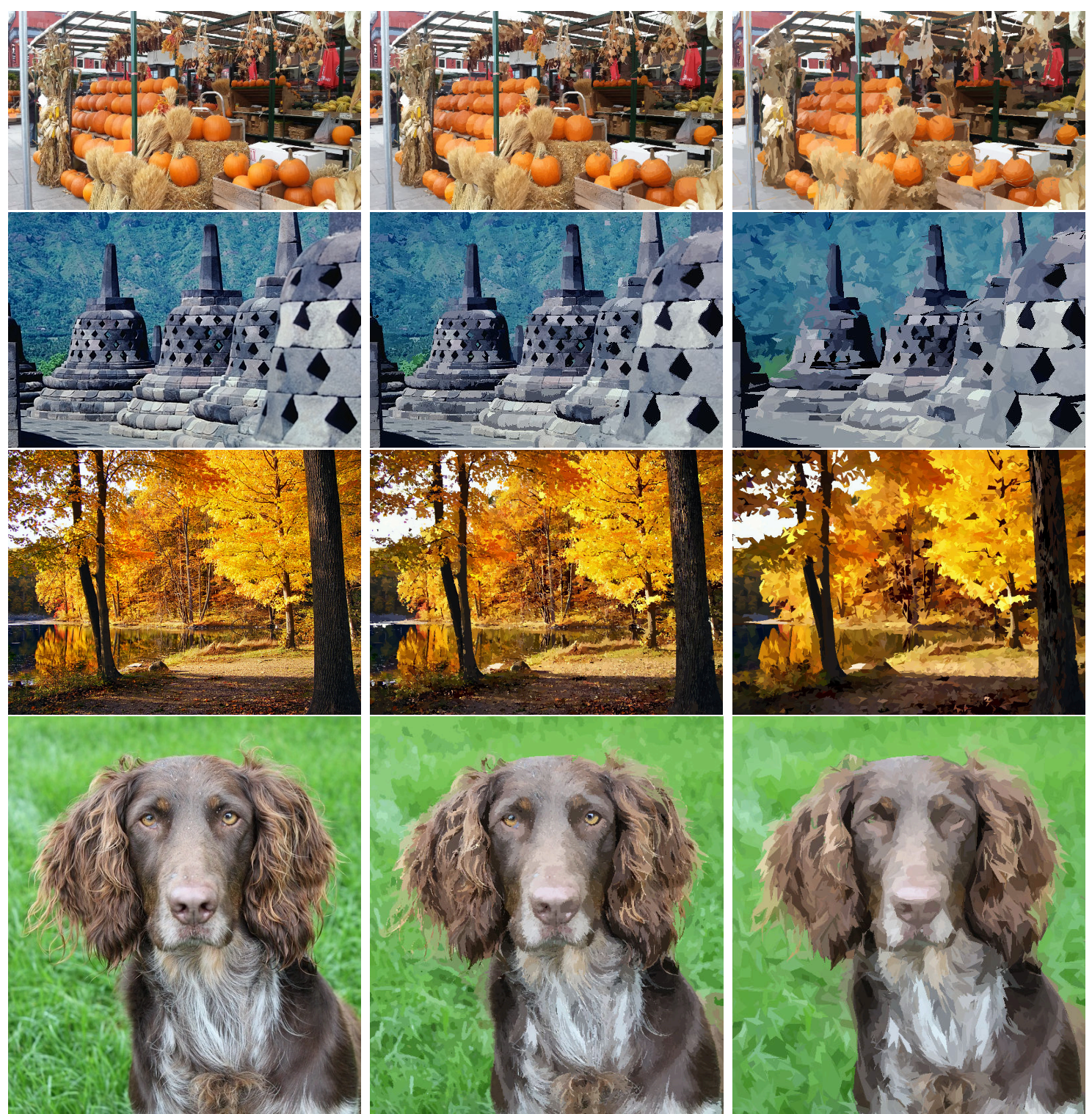

Figure 3.13: Image abstraction results. Left to right: the original images, abstractions with flattened regions and regions with boundary simplification using RDP algorithm [36]. Top to bottom: market, temple, autumn, and dog. 

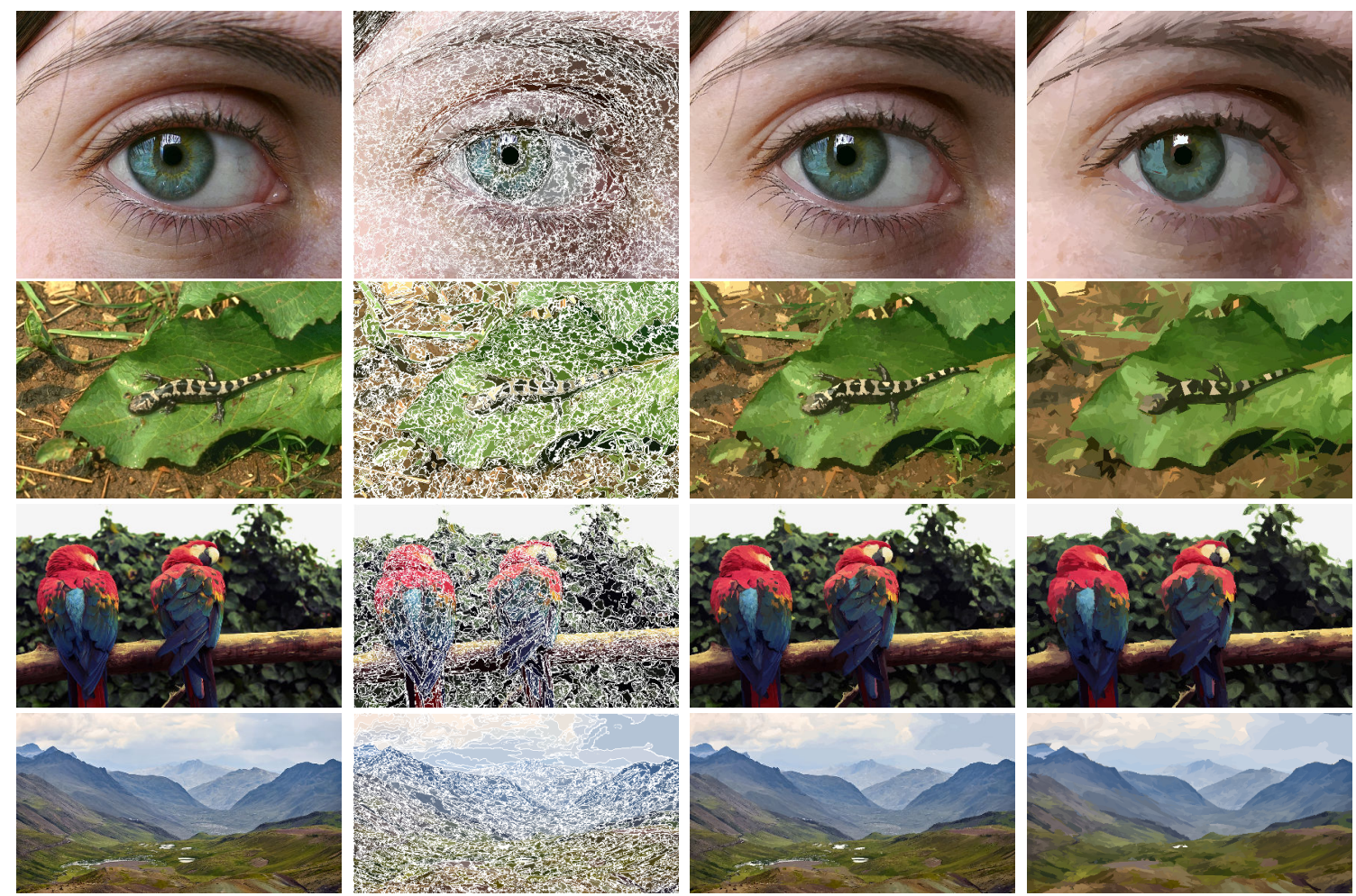

Figure 3.14: Image abstraction results (set2). Left to right: the original images, contours after flattening, abstractions with flattened regions and regions with boundary simplification using RDP algorithm [36]. Top to bottom: eye, lizard, parrots, and mountains. 
Images of nature such as the autumn image often contain irregular features like masses of leaves and bark textures. Our abstraction mechanism can keep the complexity of these features. The abstraction with simplified region boundaries gives a more severe stylization that nonetheless conveys the content of the image. Unlike the temple image, where directionality of features generated a slight undesirable effect, the less structured textures of autumn remain comprehensible.

Our abstraction retained the curvy shapes of the hair layers on the dog's ears and chest in the dog image. The background grass is out of focus and the original image is blurred. However, with abstraction, the area became more interesting textures. In the rightmost abstractions, we eliminated small regions, which prevented the eyes of the dog from being rendered. A user-supplied detail mask would fix this problem, though the method would no longer be strictly automated.

Some additional example results can be found in Figure 3.14, including the region contours.

In our examples, the regions were colored using original image colors. Modification of colors can generate artistic images as well. Some abstraction methods like artistic thresholding [140] used meanshift [25] to segment the image. For our black and white results in Figure 3.17, we used thresholding of our oversegmentation. Since this naïve method does not consider the connectivity of the regions, the effectiveness of the result is based strictly on the complex shapes of the regions we provided.

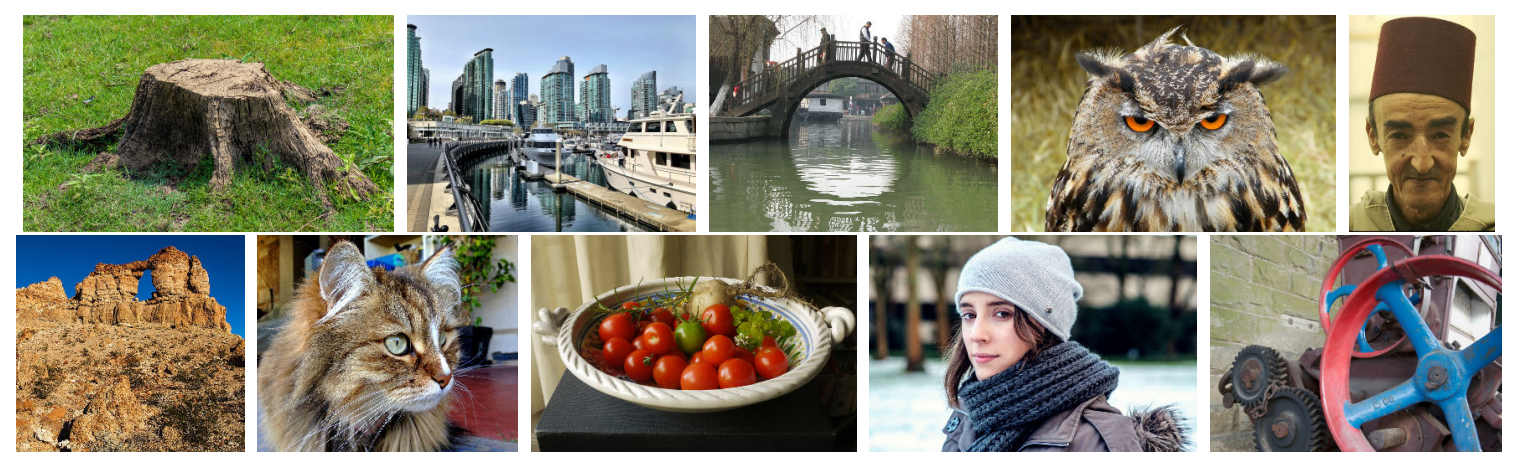

Figure 3.15: Original images of stump, port, bridge, owl, man, arch, old man, tomatoes, toque, and machinery. 


\subsubsection{Comparison with Oversegmentation Methods}

Our priority-based overlapping region creation allows us to preserve small structures. We briefly compare our segmentation with those created by SLIC [3], Felzenszwalb's method [45] and QuickShift [131]. SLIC was specifically intended to create compact, uniform-size segments; QuickShift has more variation in region size and its segments are not necessarily compact, but it still struggles to capture thin structures. The size and number of segments can vary in Felzenszwalb's method, depending on local contrast.

Figure 3.16 shows oversegmented regions produced by SLIC, QuickShift, Felzenszwalb's method, and our method. In general, our regions are far more irregular, a property undesirable for some applications but useful in stylization. Our method is capable of keeping directional patterns. The regions on the water are oriented parallel to the gradient. In the SLIC and QuickShift methods, the vertical boundaries cut across the shadows on the water and the curve on the arch of the bridge. Felzenszwalb's segmentation generates regions quite similar qualitatively to ours. For this application, many of the differences are unimportant. Still, Felzenszwalb's regions do not capture well the large structures such as the arch of the bridge. Our segmentation provides more definition in regions with gradients and small details; for example, see the branches and the area beneath the bridge.

\subsubsection{Reduced Color Palettes}

We modified the regions' colors, restricting them to small color palettes. The smallest possible color palette is a black and white palette; see Figure 3.17, where we threshold each region separately. Despite the simplicity of the approach, the oversegmentation lets us capture small details such as the fur in the cat image and thin structures in the port image.

Choosing a color palette and automatically assigning colors to regions in general is not trivial. Here, we show a simple technique, recoloring the regions with a color palette extracted from an input image. The main colors are determined using k-

means; the output color for a particular region is the nearest palette color to the region's average color. A sample result is given in Figure 3.18. The technique is a generalization of thresholding to an arbitrary palette. In the next chapter, we will 

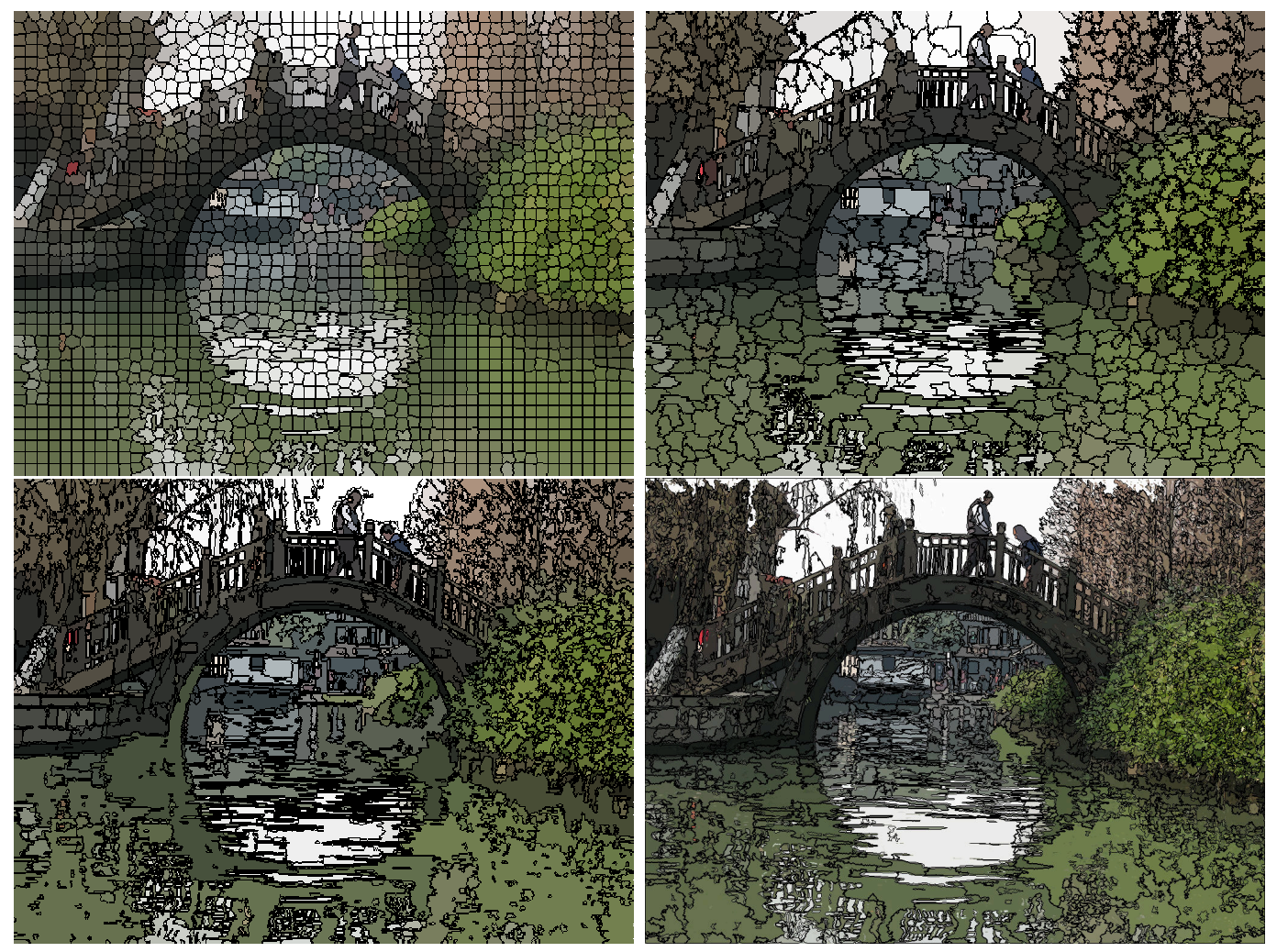

Figure 3.16: Region comparison. Left to right: regions from SLIC [3], QuickShift [131], Felzenszwalb [45], and our method.

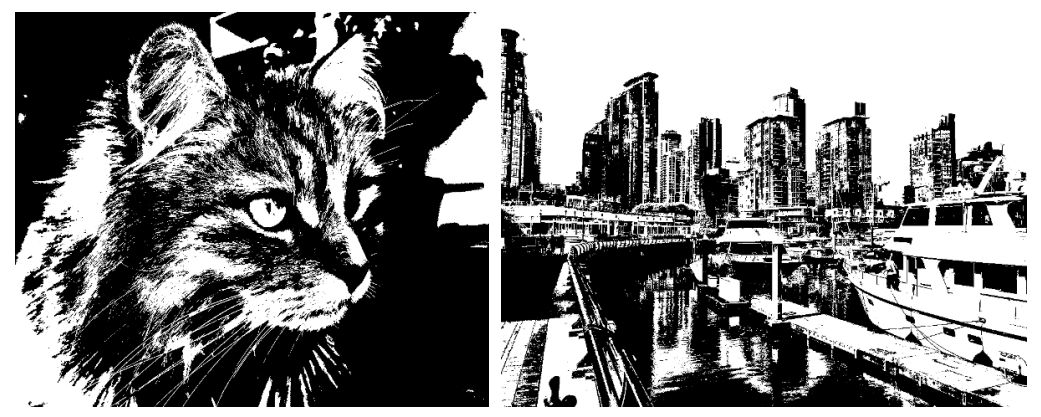

Figure 3.17: Cat and port in black and white. 
introduce a method for artistic recoloring of region-based abstraction.
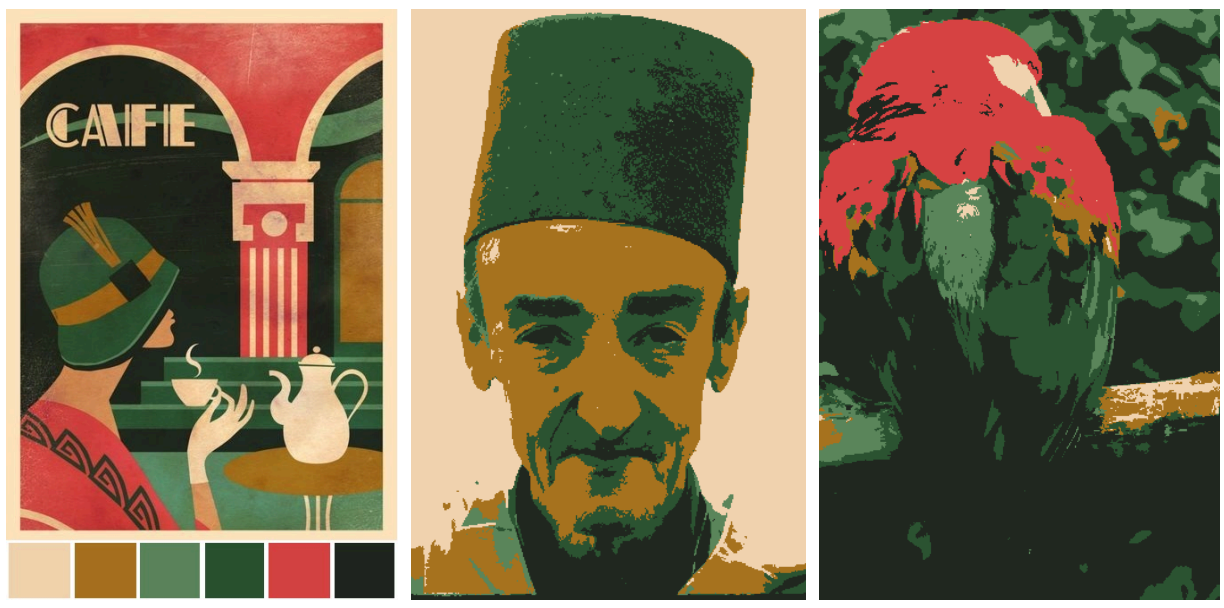

Figure 3.18: Recoloring using a color palette from Art Deco Poster. Left to right: Art Deco poster and extracted color palette; recolored old man; and parrot.

\subsubsection{Performance}

Our algorithm was implemented in $\mathrm{C}++$, using OpenCV for some functionality. On an Intel(R) Core(TM) i7-6700 with a $3.4 \mathrm{GHz}$ CPU and 16.0 GB of RAM, our unoptimized research code takes approximately 67 seconds to process a $1024 \times 680$ input image with about 4200 initial seeds, while the processing time for an image of size $480 \times 320$ with 1200 seeds is about 5 seconds. The majority of the computational expense is incurred by the region growth process.

\subsubsection{Limitations}

We intended our algorithm to create highly complex regions, making use of local image detail to guide region shapes. However, it works best when there is significant local color variation. When applied to blurred or smooth images, the lack of detail can lead to more uniform region shapes. For example, consider the blurred background in the toque image and the smooth surfaces in the tomatoes image, shown in Figure 3.19. 

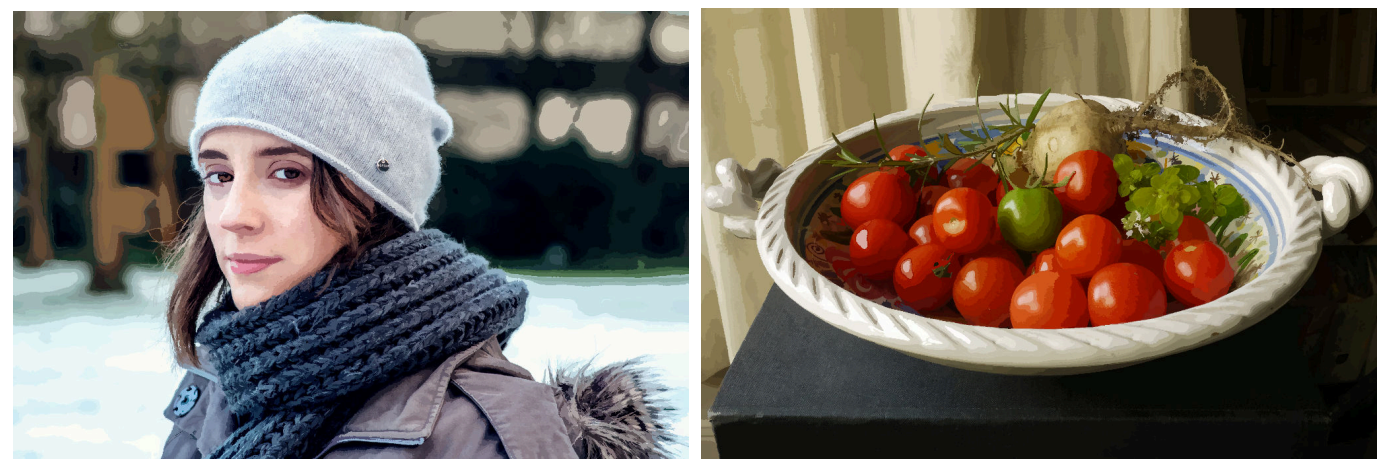

Figure 3.19: Uninteresting region shapes in the blurred background of toque and smooth surfaces on tomatoes.

\subsection{Summary}

In this chapter, we presented an image stylization technique that creates intricate spatial primitives using a region growth mechanism, customized to the target image, then covers the image plane with these primitives. The results strike a balance between detail removal and preservation. Further abstraction is possible through simplifying the region boundaries and recoloring the regions. We have obtained appealing abstractions using the cumulative range geodesic, and presented some preliminary results of manipulating these boundaries and coloring the regions.

This work has been published as follows [9]:

- Rosa Azami and David Mould. "Image abstraction through overlapping region growth," In Proc. Graphics Interface (2020), pp. 66 - 73. 


\section{Chapter 4}

\section{Recoloring of Region-based Abstraction}

\subsection{Introduction}

This chapter presents a recoloring approach that assigns vivid colors to the regions obtained from our region-based abstraction method in the previous chapter. Often time, averaging of the colors through an abstraction process would turn an image dull and uninteresting. Color plays an important role in image aesthetics. In representational art, artists employ colors that match the perceived colors of objects in the depicted scene. Conversely, abstraction provides more freedom to manipulate colors. Figure 4.1 shows a modern vector illustration of a lion, an example of Fauvism by André Derain, and an abstraction of the Eiffel Tower by Robert Delaunay. The artists have expressed the image content by vivid colors assigned carefully various image regions. We aim at a method that can generate colorful images, recoloring an image using an arbitrary input palette. In this chapter, we describe a method for recoloring an image based on a subdivision of the image into distinct segments, recoloring each segment based on its relationship with its neighbours.

Our goal is to present different recoloring possibilities by assigning colors to each region of an oversegmented input image. We aim to maintain the image contrast and preserve strong edges so that the content of the scene remains recognizable. It is important to convey textures and small features, often too delicate to be preserved by existing abstraction methods. We would like to be able to create wild and vivid abstractions through use of unusual palettes.

Manual recoloring of oversegmented images would be tedious; the images contain hundreds or thousands of segments; clicking on every segment would take a long time, 

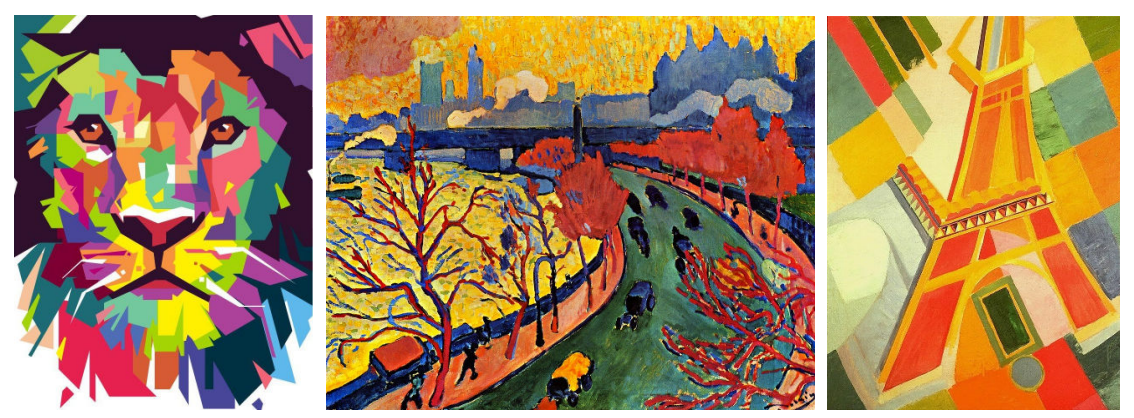

Figure 4.1: Colorful representational images. A modern vector illustration of a lion; a Fauvist painting by André Derain; an abstraction of the Eiffel Tower by Robert Delaunay.

even leaving aside the cognitive and interaction overhead of making selections from the palette. We provide an automatic assignment of colors to regions. The assignment can be used as is, could be used in a fast manual assessment loop (for example, if an artist wanted to choose a suitable palette for coloring a scene), or could be a good starting point for a semi-automatic approach where a user made minor modifications to the automated results.

In this chapter, we present an automatic recoloring approach for a region-based abstraction. The input is a desired palette and an oversegmented image. The method assigns a color from the palette to each region; it is based on the widest path algorithm [109], which organizes the regions into a tree based on the weight of the edges connecting them. We use color differences between adjacent regions both to order the regions and to select colors, trying to match the difference magnitude between the assigned palette colors and the original region colors. The use of color differences allows structures in the image to remain recognizable despite breaking the link between the original and depicted color.

The chapter's main contributions are as follows:

- We designed a recoloring method for an oversegmented image, creating multiple abstractions colored with just one palette. Our method creates wild and high contrast images.

- Various styles can be created by our method. We experiment with color blending between regions and produce smooth images. By reducing the palette colors, we simplify the recolored images. Moreover, we generate new colorings from a palette by applying different metrics and color spaces. 


\subsection{Recoloring Algorithm}

We present a recoloring algorithm that automatically assigns colors to regions of an oversegmented image. Our system takes as input a set of regions and a palette containing a set of colors and assigns a color to each region. The recolored image should convey recognizable objects in the image. Edges are essential to the visibility of the structures. Neighboring regions will be assigned distinct colors to express an edge, and regions of similar colors will be assigned similar colors. The human visual system is sensitive to brightness contrast; to help preserve contrast in our recoloring, we take into account the regions' relative luminance changes when selecting region colors.

We take the strategy of emphasizing color differences between regions, seeking to match the original color distance value between adjacent regions without preserving the colors themselves. Neighbouring regions with large differences will be assigned distant colors, preserving the boundary, while similar-colored regions will be assigned similar output colors or even the same color.

We use a graph structure to organize the segmented image, where each region is a node and edges link adjacent regions. To simplify color assignment, we will construct a tree over the graph, with a subsequent tree traversal assigning colors to nodes based on the color of the parent node.

One possible approach to color assignment would use direct optimization. A suitable optimization framework for our case would take as input the graph, the palette, and suitable region descriptors such as average region color; it would then assign to each region a color from the palette, seeking to minimize an energy function over the labeling. Given an energy function, various minimization techniques exist and could be deployed straightforwardly.

However, designing an energy function for a particular goal is in general challenging. In our case, it is unclear what the energy function should look like. We want both flexibility in assigning a wide range of colors to the regions, and preservation of visual content in the original image. Our strategy of maintaining color difference magnitudes between adjacent nodes succeeds at the latter part, but when enacted over the entire adjacency graph, would not satisfy the former consideration. We suspect that instead it would color regions of similar input color with constant output color, contrary to our intention.

Our proposed approach is to break the adjacency graph into a tree, assigning 
colors over tree traversal. This strategy increases opportunities for unusual color transitions across unimportant region boundaries (neighbor regions that belong to different branches), allowing irregular recolorings as in the inspirational artwork of Figure 4.1. We assign weights to the edges reflecting their priority, with large regions, regions of very similar color, and regions of very different color receiving high priority. Both small regions and regions with intermediate color differences receive lower priority. Once weights have been assigned, we find the tree within the graph that maximizes the weight of the minimum-weight edges, a construction that corresponds to the widest path problem.

Our algorithm has two main steps. First, we create a tree by applying the widest path algorithm [109] on the region graph. Then we assign colors to regions by traversing the tree beginning from its root. For each region in the graph, we choose the color from the palette that best matches the color difference with its parent. Before starting, we apply histogram matching between the regions' color differences and the palette color differences. The histogram matching allows us to best convey the image content while using the full extent of an arbitrary input palette, even one that has a color distribution very different from that of the input image.

We show a flowchart of our recoloring approach in Figure 4.2. The input is (a) an oversegmented image and (b) a palette to use in the recoloring. We compute (c) the adjacency graph of the segments and aggregate the differences between adjacent colors into the set $\{\Delta Q\}$. The widest path algorithm (d) gives us a tree linking all nodes of the adjacency graph. We next compute (e) the set of differences between palette colors, yielding $\{\Delta P\}$. We match the histogram (f) of $\{\Delta Q\}$ to that of $\{\Delta P\}$ and $(\mathrm{g})$ assign colors to all regions by traversing the widest-path tree, resulting in $(\mathrm{h})$ the fully recolored image.

\subsubsection{Tree Creation from Widest Path}

Before we explain the recoloring proper, we will describe tree creation. We will employ the widest path algorithm to create a tree over the input oversegmentation. Later, we will traverse the tree and assign a color to each region, matching the edge's target color difference with the color difference with its parent's color. In practice, it is possible to combine the tree creation and traversal, since the widest-path algorithm involves a best-first traversal of the tree as it is being built. Prior to color assignment, 


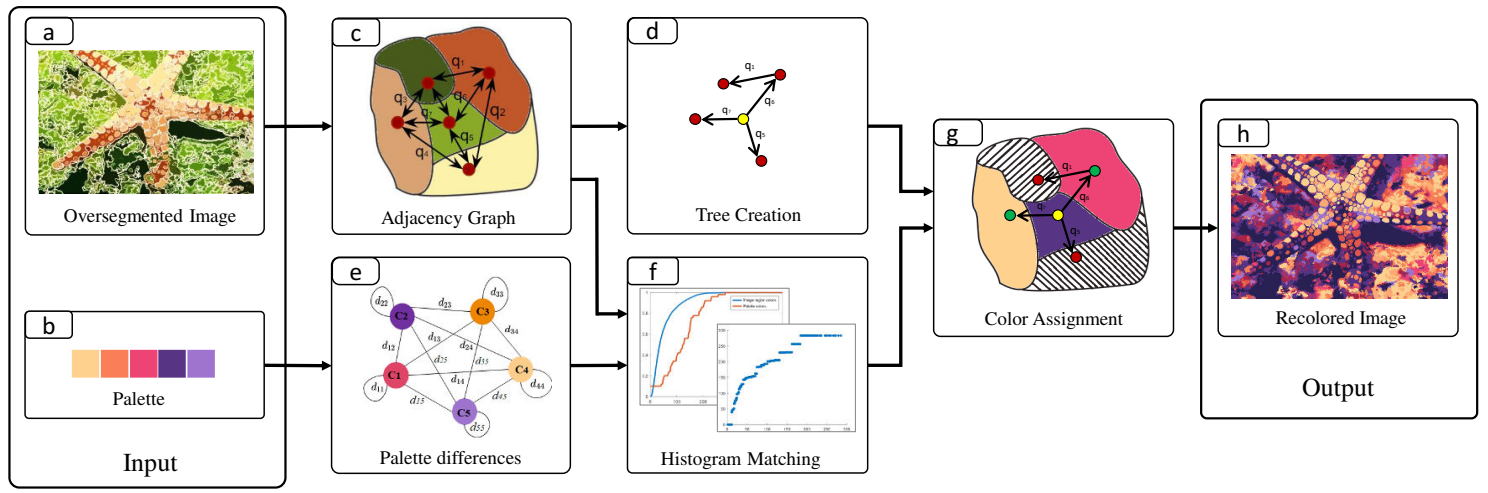

Figure 4.2: Recoloring algorithm pipeline.

we apply histogram matching to align the regions' color differences with the palette's for better use of palette colors.

\subsubsection{Tree Creation: The Widest Path Problem}

Pollack [109] introduced the widest path problem. Consider a weighted graph consisting of nodes and edges $G=(V, E)$, where an edge $(u, v) \in E$ connects node $u$ to $v$. Let $w(u, v)$ be the weight, called capacity, of edge $(u, v) \in E$; capacity represents the maximum flow that can pass from $u$ to $v$ through that edge. The minimum weight among traversed edges defines the capacity of a path. Formally, the capacity $C(u, v)$ of a path between nodes $u$ and $v$ is given by the following:

$$
C(u, v)=\min (w(u, a), w(a, b), \ldots, w(d, v))
$$

where $w(u, a), w(a, b), \ldots, w(d, v)$ are the edge weights along the path. The widest path between $u$ and $v$ is the path with the maximum capacity among all possible paths.

In a single-source widest path problem, we calculate for each node $t \in V$ a value $B(t)$, the maximum path capacity among all the paths from source $s$ to $t$. The value $B(t)$ is the width of the node. The union of widest paths from the source to each node is a tree, which we use to order the color assignment process. We can choose any node as the source; our implementation uses the region containing the image centre.

The widest path algorithm can be implemented as a variant of Dijkstra's algorithm, building a tree outward from the source node $s$ to every node in the graph. All nodes of the graph $t \in V$ are given an initial width value; the source node $s$ will be assigned $B(s)=+\infty$ and all other nodes $v \neq s$ will have $B(v)=-\infty$. A 
priority queue holds the nodes; at each step of the algorithm, we take the node with the highest current width from the queue and process it, stopping when the queue is empty. Suppose the node $u$ is on top of the queue with a width $B(u)$. For every outgoing edge $(u, v)$, we update the value of the neighbour node $v$ as follows:

$$
B(v) \leftarrow \max \{B(v), \min \{B(u), w(u, v)\}\}
$$

where $w(u, v)$ is the edge weight between nodes $u$ and $v$. If the value $B(v)$ was changed, node $u$ will be set as its parent node and $v$ will be added to the queue. When the algorithm terminates, all non-root nodes in the graph will have been assigned a parent, thus providing a tree rooted at $s$.

In our application, one possibility for edge weight is to use the difference in color values between the two regions. This would ensure that the widest path tree linked dissimilar regions, resulting in good edge preservation. However, regions of similar color could easily be divided. We want to preserve small color distances as well, so we need to assign a large edge weight to small color differences. Distances intermediate between large and small are of the least importance. Hence, we base our edge weight on the difference from the median color distance, as follows.

We calculate the color distances across each edge in the adjacency graph; call the set of color distances $\{\Delta Q\}$, with

$$
\{\Delta Q\}=\left\{D_{c}\left(c_{i}, c_{j}\right)\right\}=\left\{\Delta q_{i j}\right\}, \quad i \neq j, \quad r_{i}, r_{j} \in R
$$

where $c_{i}$ and $c_{j}$ are the colors of regions $r_{i}$ and $r_{j}$, and $D_{c}$ is the function computing the color distance. Compute the median value $\Delta \bar{q}$ from the distances in $\{\Delta Q\}$.

We also want to take into account the size of the region, such that larger regions have greater importance; we prefer that a larger region have higher priority and thus influence the smaller regions that are processed afterwards, compared to the converse. Depending on the oversegmentation, action may not be necessary; the process is intended to improve results on oversegmentations with a dramatic variation in region size.

We compute for each region a factor $b$, the ratio of the region's size (in pixels) to the average region size. Then, when we traverse an edge, we use the $b$ of the destination region to determine the weight. In our implementation, we compute and store a single edge weight; there is no ambiguity about the factor $b$ because we only 
ever traverse a given edge in one direction, moving outward from the source node.

To summarize: when traversing an edge, the edge weight is the distance between its target color difference and the median color difference, multiplied by a factor of $(1+b)$ for the destination region:

$$
w\left(r_{i}, r_{j}\right)=(1+b)\left|D_{c}\left(c_{i}, c_{j}\right)-\Delta \bar{q}\right| .
$$

The factor $(1+b)$ takes size into account, but ensures the region's color differences can still affect the traversal order even for very small regions ( $b$ near zero). Note that the function $D_{c}$ depends on the colorspace used. A simple possibility is Euclidean distance in RGB, but more perceptually based color distances are possible. We discuss color distance metrics in section 4.4.2.

\subsubsection{Histogram Matching}

We plan to match color differences in the output to the color differences in the input. However, the input palette can have an arbitrary set of colors, and we want to make use of the full palette, which might not happen with direct matching. For example, imagine a low-contrast image recolored with a palette of more varied colors. The smallest palette difference might be quite large; if so, the muted areas of the original will be matched with difference zero, resulting in loss of detail in such regions. A narrow palette applied to a high-contrast image will have similar problems in the opposite direction.

To adapt the palette usage to the input image color distribution, we apply histogram matching to color differences. We emphasize that we are not matching the colors themselves, but the distributions of differences. Histogram matching is applied between the region color differences (the distribution of values in $\{\Delta Q\}$, computed in Section 4.2.2) and the pairwise color differences of the palette (call this dataset $\{\Delta P\})$.

The histogram matching gives a new target color difference for each graph edge; call this target $\Delta q^{\prime}(u, v)$ for the edge linking regions $u$ and $v$. The matching ensures that the distribution of values $\left\{\Delta q^{\prime}\right\}$ is the same as the distribution of values in $\{\Delta P\}$. The values $\Delta q^{\prime}$ are then used for color assignment, selecting color pairs from the palette which correspond to the same place in the distribution: medium palette differences where medium image color differences existed, small differences where the 
original image color differences were small, with the largest palette differences reserved for the largest differences in the original image. The idea is illustrated in Figure 4.3, which shows an example of cumulative distribution functions for the original region color differences, the palette color differences, and the region color differences after matching. In this case, the image had smaller differences than the palette, so the target region differences are heightened.

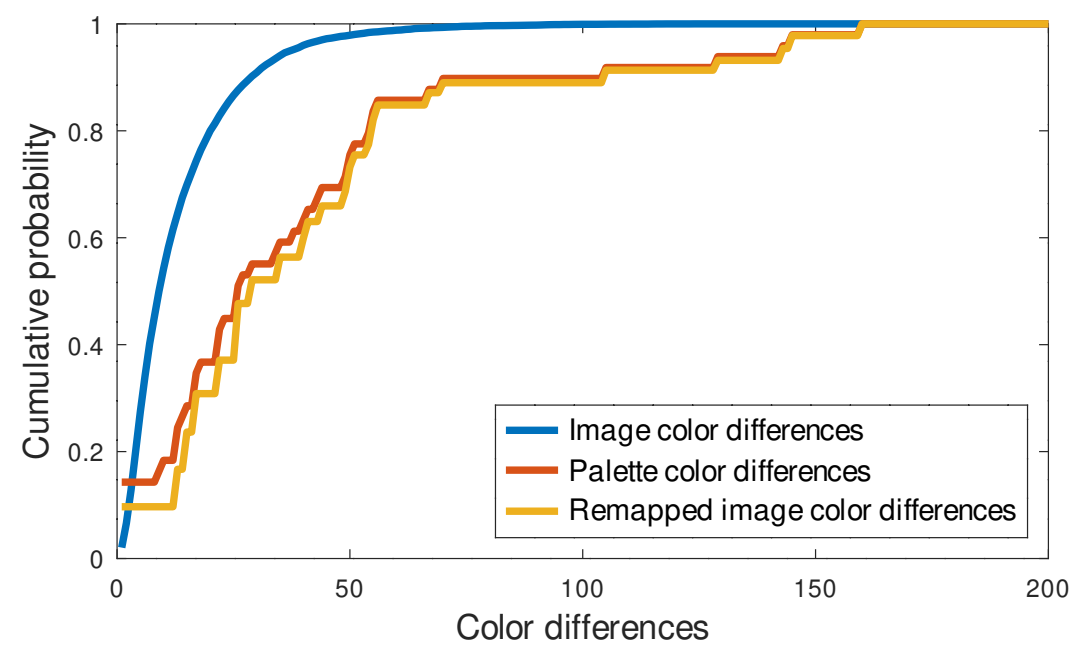

Figure 4.3: Histogram matching color distances: cdfs of the original image $\{\Delta Q\}$ (blue), the palette $\{\Delta P\}$ (red), and the target color differences $\left\{\Delta q^{\prime}\right\}$ (yellow).

Figure 4.4 shows the input image with average region colors and the recolored images before and after histogram matching. The bar graphs under each image show the proportion of each color in the image. We can see that the histogram matching used the palette colors more evenly, increasing contrast and highlighting more details. For example, strong edges on the leaf boundary became distinguishable from the nearby regions, and the markings on the lizard became more prominent.

\subsubsection{Color Assignment}

The widest path algorithm provided a tree, and the histogram matching provided palette-customized target distances for the edges. We now traverse the tree and assign a color to each region along the way. We begin by assigning the closet palette color to the tree's root node; recall that the root was the most central region in the image. At each subsequent step, we assign a color from the palette $P$ to the 

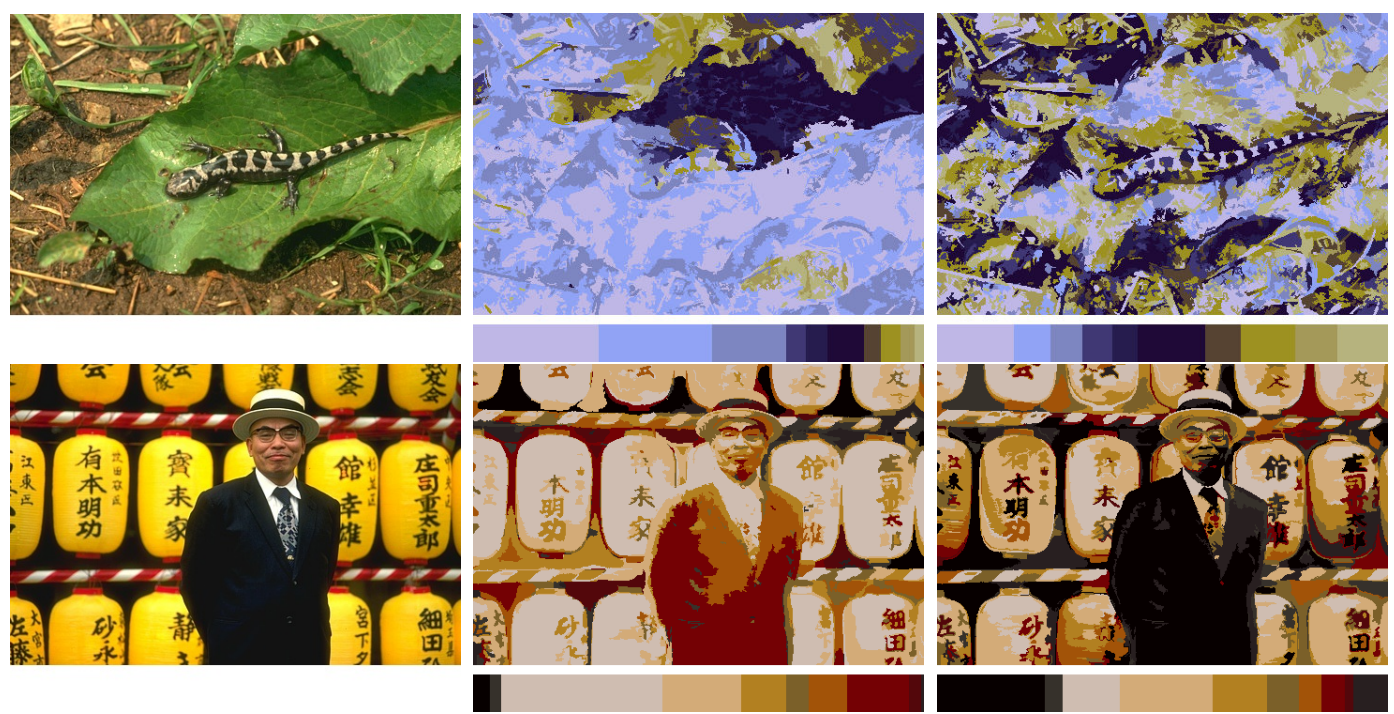

Figure 4.4: Histogram matching result. Left to right: original image, result without histogram matching, and result using histogram matching.

current region $\alpha$ based on the palette color $p_{\beta}$ already assigned to the parent region $\beta$ and the target color difference $\Delta q^{\prime}(\alpha, \beta)$. We also consider the luminance difference between regions $\alpha$ and $\beta$ so as to help maintain larger-scale intensity gradients. Recall our intention in preserving color differences: two regions with large color differences should be assigned two very different colors, and regions with a small color difference should get very similar colors, possibly the same color. Owing to histogram matching, "large" and "small" are calibrated to the content of the particular image and palette being combined.

We impose a luminance constraint on potential palette colors, in an effort to respect the relative ordering of the regions' luminances. Suppose the luminances of two regions $\alpha$ and $\beta$ are $L_{\alpha}$ and $L_{\beta}$, where $L_{\alpha}<L_{\beta}$. We then constrain the set of eligible palette colors for region $\alpha$ such that only colors $p_{\alpha}$ that satisfy $L_{p_{\alpha}}<L_{p_{\beta}}$ are considered. A similar constraint is imposed if $L_{\alpha}>L_{\beta}$.

For region $\alpha$ and its parent $\beta$, we have the target edge difference $\Delta q^{\prime}(\alpha, \beta)$. Denote by $p_{\beta}$ the palette color already assigned to the parent region $\beta$. We choose the palette color $p_{\alpha}$ for region $\alpha$ so as to minimize the distance $D$ :

$$
D=\left|\Delta q^{\prime}(\alpha, \beta)-D_{c}\left(p_{\alpha}, p_{\beta}\right)\right|
$$

where $D_{c}$ is the distance metric between two colors. The only colors considered for 
$p_{\alpha}$ are those that satisfy the luminance constraint.

For colors with the lowest and highest luminance in the palette, there may be no available colors satisfying the luminance constraint. In such cases, the constraint is ignored and all palette colors are considered.

Since the source region has no parent, the above process can not be used to find its color. Instead, we assign the closest color from the palette, as determined by the difference metric $D_{c}$. The source region itself is the region containing the centre of the image; while the output is weakly dependent on the choice of starting region, we do not view the starting region as a critical decision. Figure 4.5 shows some examples of varied outcomes from moving the starting region.
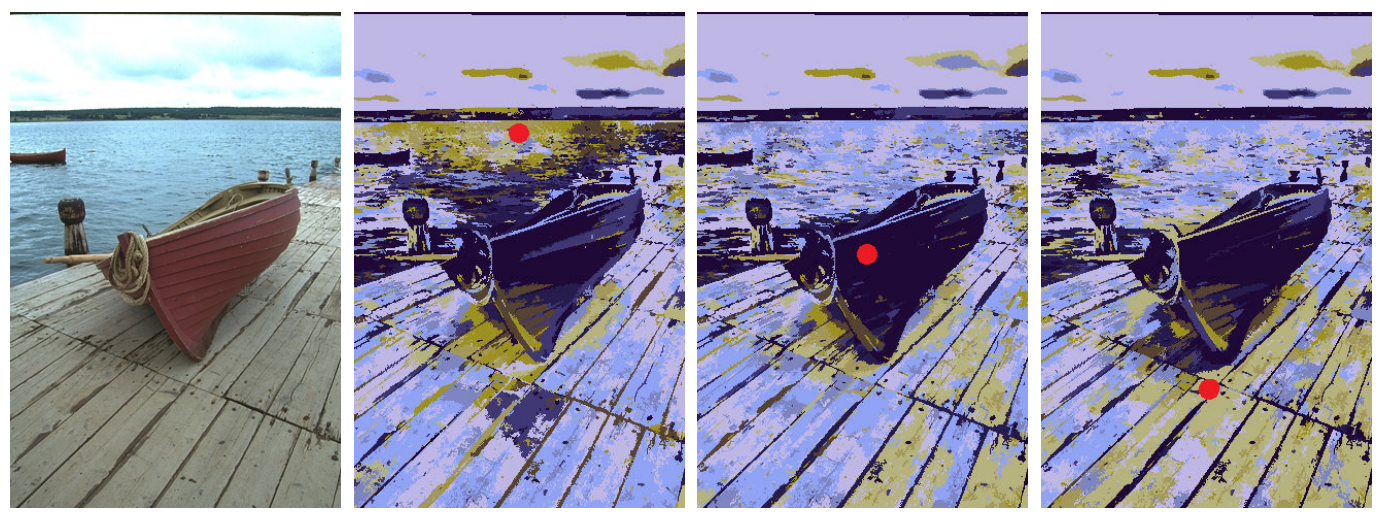

Figure 4.5: Changing the source region locations. The starting region is indicated by a dot.

\subsection{Results and Discussion}

In this section, we present some results. Figures 4.6 and 4.7 show a variety of recolored images generated by our algorithm using various palettes. We succeeded in maintaining strong edges, and objects in the recolored abstractions remain recognizable. Our algorithm retains textures and produces vivid recolored images by selecting varied colors from the palette. It assigns the same colors over flat regions and distinct colors to illustrate structures. We ran our algorithm on a variety of images with different textures and contrasts. We obtained most of our palettes from the website COLRD (http://colrd.com/); others we created manually by sampling from colorful images. 


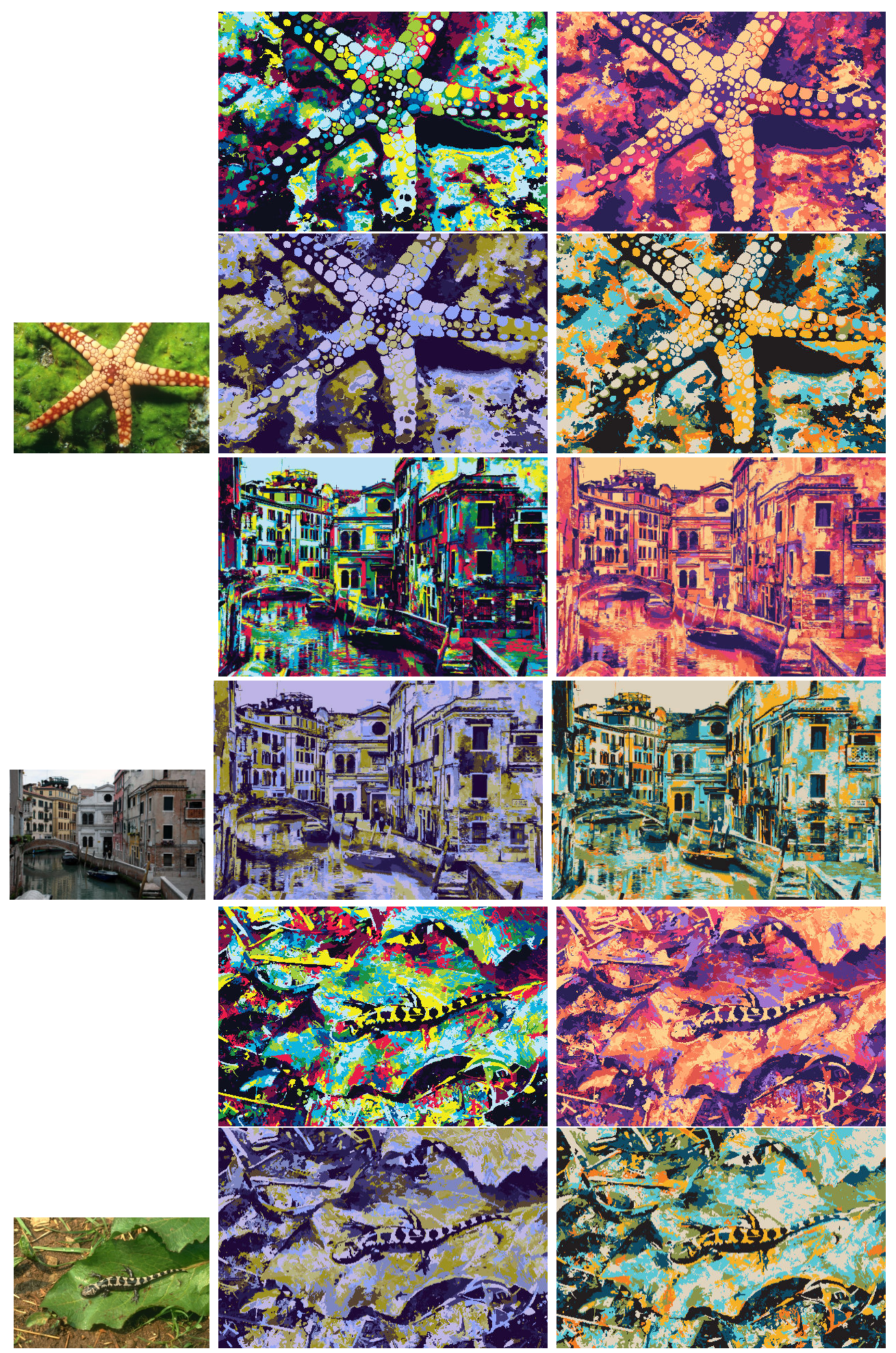

Figure 4.6: Region recoloring results. Images, top to bottom: starfish, Venice, and lizard. 


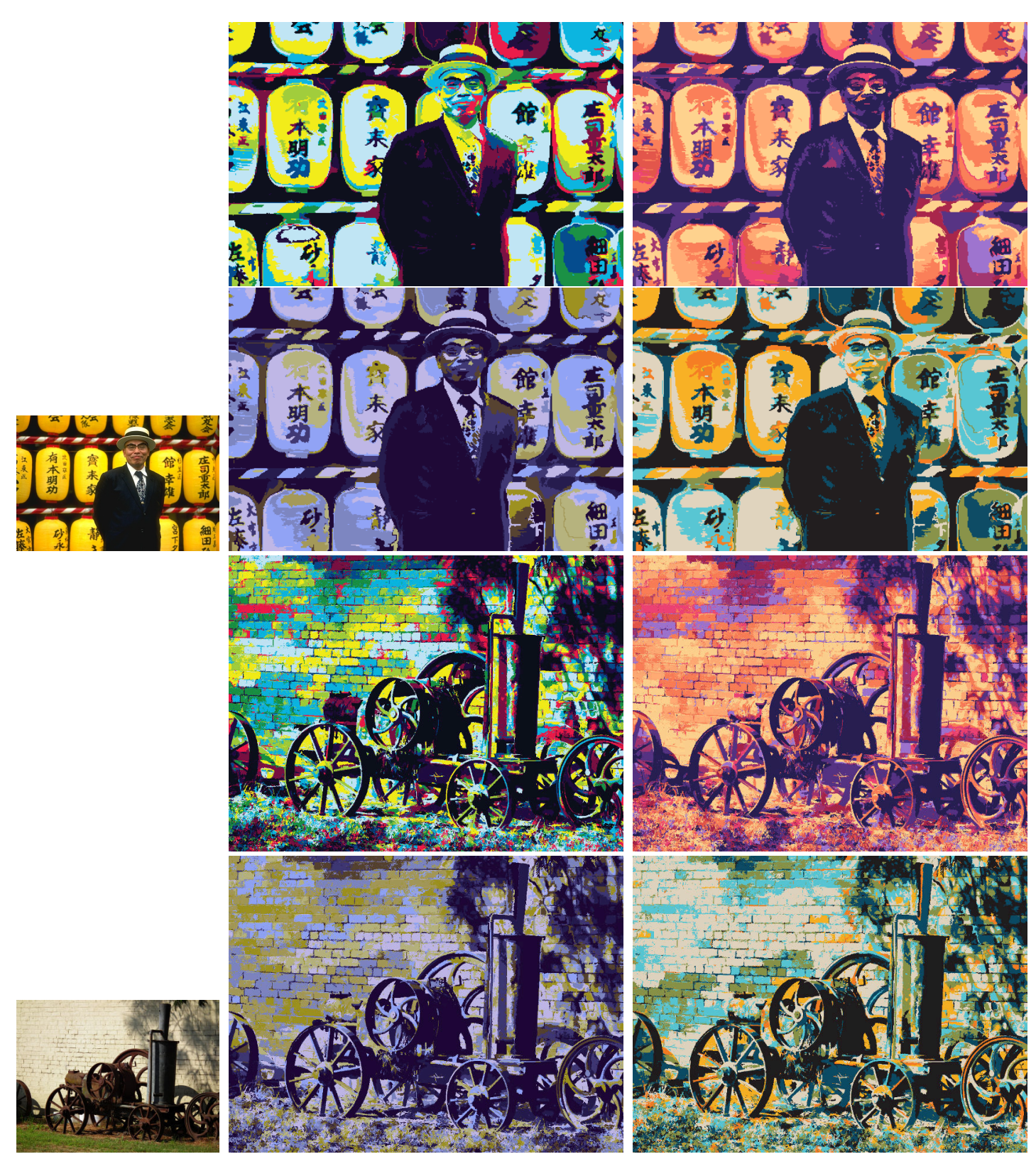

Figure 4.7: Region recoloring results. Images: lanterns and rust. 
In Figures 4.6 and 4.7, we present a set of examples from our recoloring algorithm, which were generated with four different palettes. From left to right, the columns show the original image, followed by recolored images using different palettes. We chose images presenting different features. The delicate features and textures in the abstractions stay visible after recoloring despite the input photographs having been radically altered by the recoloring.

In the starfish image, the structure and the patterns on the arms become more prominent. The uniform colors of the background become a vivid splash of colors, emphasizing the textureness of the terrain. The algorithm has chosen the darkest colors to assign to the shadows and the lightest ones to the surface of the creature.

The Venice canal is a crowded image composed of soft textures and structures with hard edges. The algorithm is able to preserve recognizable objects such as the boats and windows. Even tiny letters on the wall and pedestrians on the canal's side are visible. The recoloring process preserved the buildings' rigid structures; meanwhile, it captured shadows and the water's soft movements. In presenting such features, adopting a highly irregular oversegmentation was necessary.

The lizard image is an example of a low-contrast image with textured areas covered by dull colors. The algorithm highlighted the textures by assigning wild colors to the homogeneous regions on the leaf. At the same time, substantial edges like the lizard's body patterns and the leaf edges are preserved naturally by our algorithm.

The next example shows a high-contrast input. The algorithm assigned the darkest colors from each palette to the coat of the man and separated it from the background using a very light color. Further, the small features on the face and the Chinese characters are mostly readable.

The rust image shows different textures on the wall and the grass, plus soft textureless areas on the machinery. The brick patterns on the wall, exaggerated in the color assignment, made the final images more interesting than the original flat image. The high-frequency details of the grass are retained. The smooth transition of colors on the top right of the image portrays the shadows.

We demonstrated strong edge preservation in all examples. Additionally, the image textures preserved, and palette colors are uniformly used to maintain a good contrast. 


\subsubsection{Comparison with Naïve Methods}

Figure 4.8 gives a comparison between our method and two naïve alternatives: closest color and random color. For "closest color", each segment is colored with the palette color closest to the segment's average color. For "random color", each segment is randomly and independently assigned a color from the palette. Using the closest color preserves some image content, but the result shows large regions of constant color; many of the palette colors are underused, an issue that can worsen when there is a significant mismatch between the original image color distribution and the palette, as in the upper example. Random assignment provides an even distribution of palette colors, but the image content can become unrecognizable for highly textured images, as in the lower example. Our method uses the palette more effectively, showing local details and large-scale content and exercising the full range of available colors. More examples of naïve recoloring are shown in Figure 4.9.
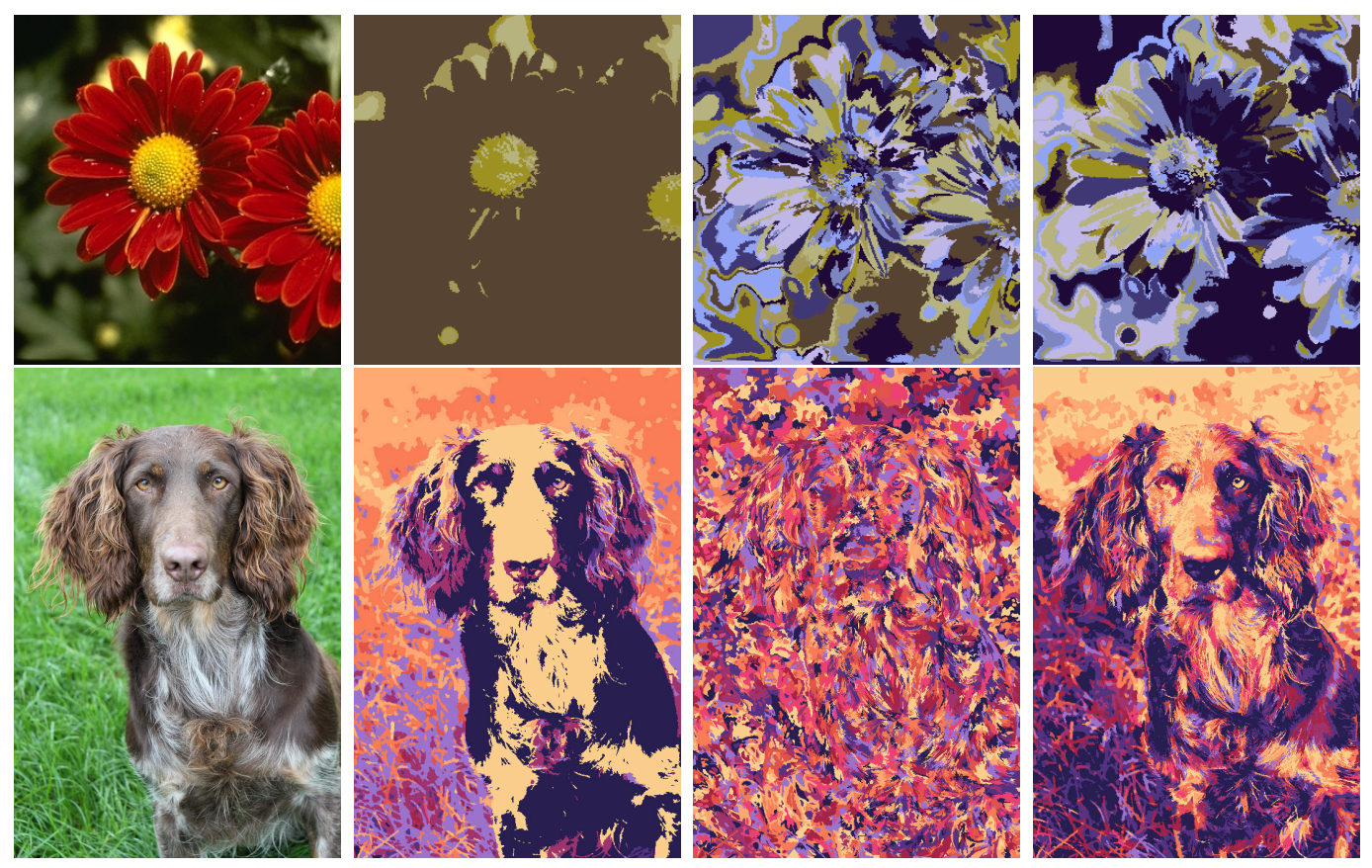

Figure 4.8: A comparison with naïve recolorings. Left to right: The original image, the results from naïve method, random recoloring, and ours. 


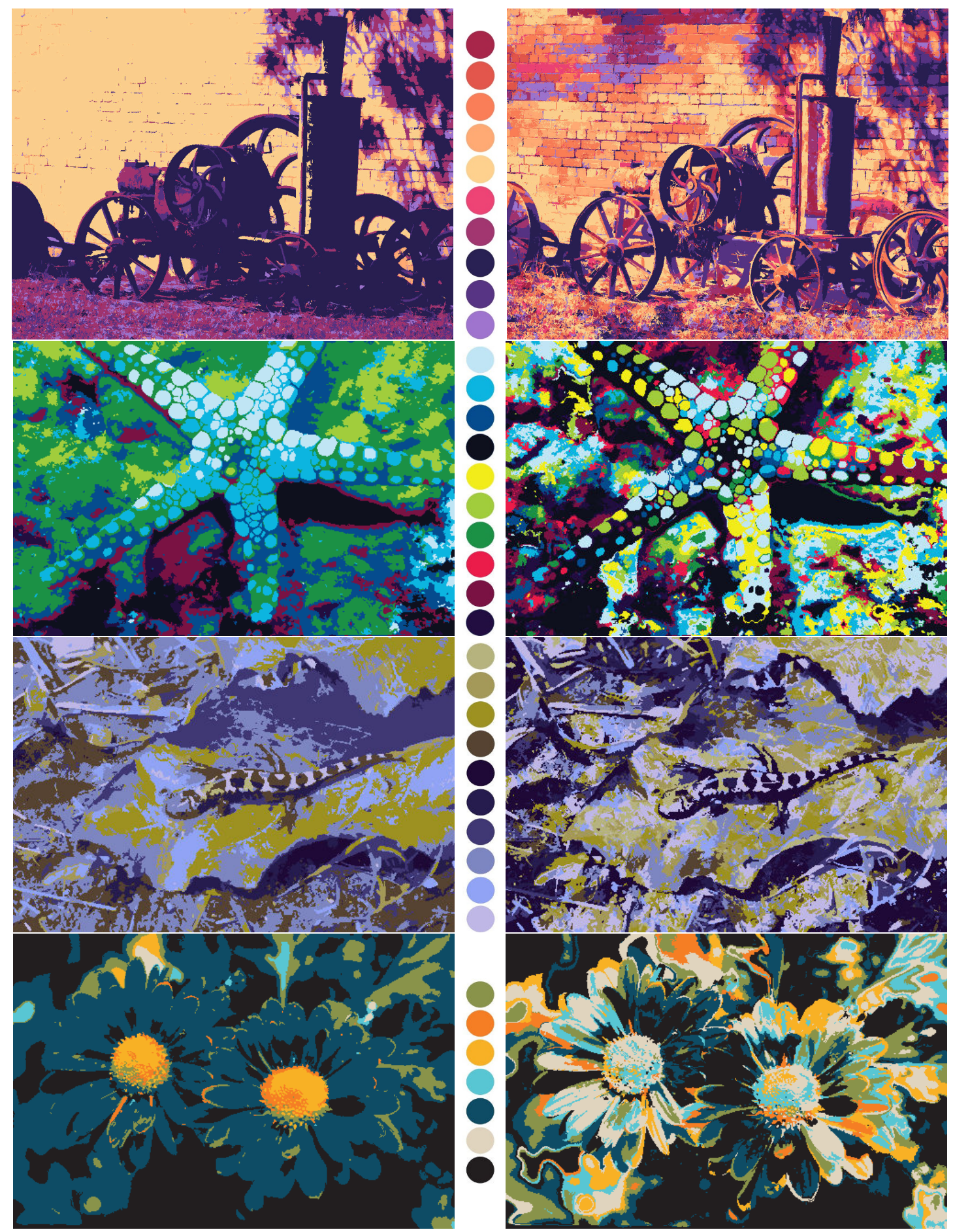

Figure 4.9: Comparing with naïve recoloring. Left: closest color assigned to regions, right: our results. 


\subsubsection{Comparison with ColorArt}

We next compare our recoloring method with ColorArt [14], an optimization-based recoloring method for graphic art. This method assigns colors to regions by solving a graph matching problem over color groups in the reference and the template image. In searching for a reference image, this algorithm uses the same number of color groups as in the template image.

Figure 4.10 shows images generated by ColorArt on the right and ours in the middle, both using the sunset palette. We created a colorful leaf surrounded by a light background as in the input image, showing the algorithm respects the changes in lightness. Moreover, assignment of different colors on the leaf presented an interesting texture. The leaf image generated by the ColorArt method has reversed the image tones. In the sketch image, we preserved the edges and showed a recognizable face in the image. In contrast, the ColorArt algorithm had difficulty with the edges and the gradual gradients, resulting in a somewhat incoherent output.

\subsubsection{Recoloring with SLIC0 Oversegmentation}

Our recoloring algorithm does not make any assumptions about the input oversegmentation. Figure 4.11 shows results from an oversegmantation from SLIC0 [2]. The starfish and owl images have approximately 2000 and 5000 segments, respectively.

Note that more irregular regions can better represent complex image contours and textures, allowing the recolored abstractions to better display the image content. In starfish, the structures and shadows are represented by distinct colors that contrast between the object and the background. The strong edges, such as the arms of the starfish, are preserved; however, the thin features are not captured by SLIC0's uniform regions, and the background terrain does not present any significant information. In the owl image, the small regions on the chest convey the feather textures, while regions such as dark eyes kept their well-defined structures. Given a suitably detailed oversegmentation, we can produce appealing results.

\subsubsection{Performance}

We ran our algorithm on an Intel(R) Core(TM) i7-6700 with a $3.4 \mathrm{GHz}$ CPU and 16.0 GB of RAM. The processing time increases with the number of regions and edges in 

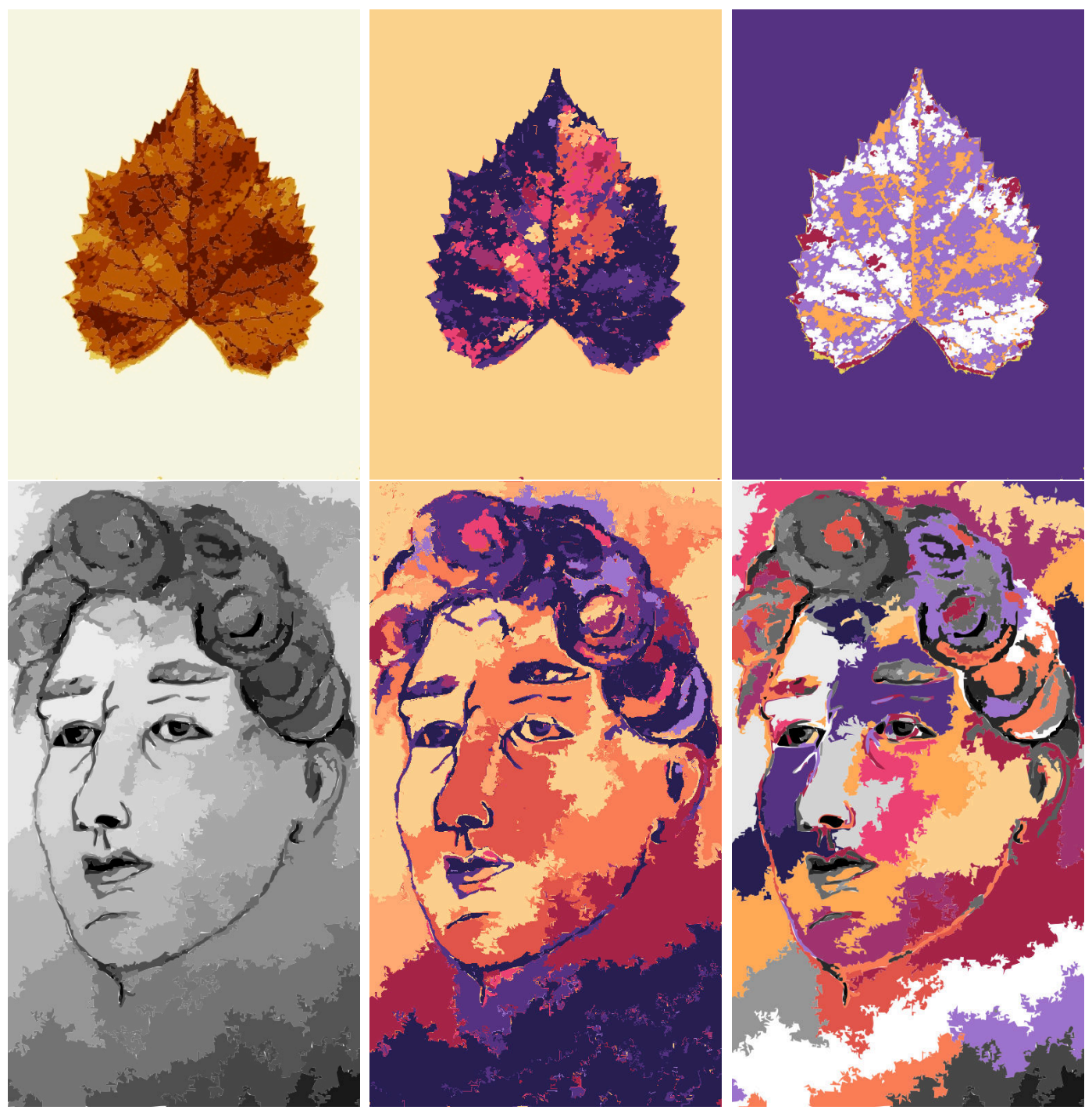

Figure 4.10: Comparison with ColorArt. Left: input; middle: our results; right: ColorArt results.

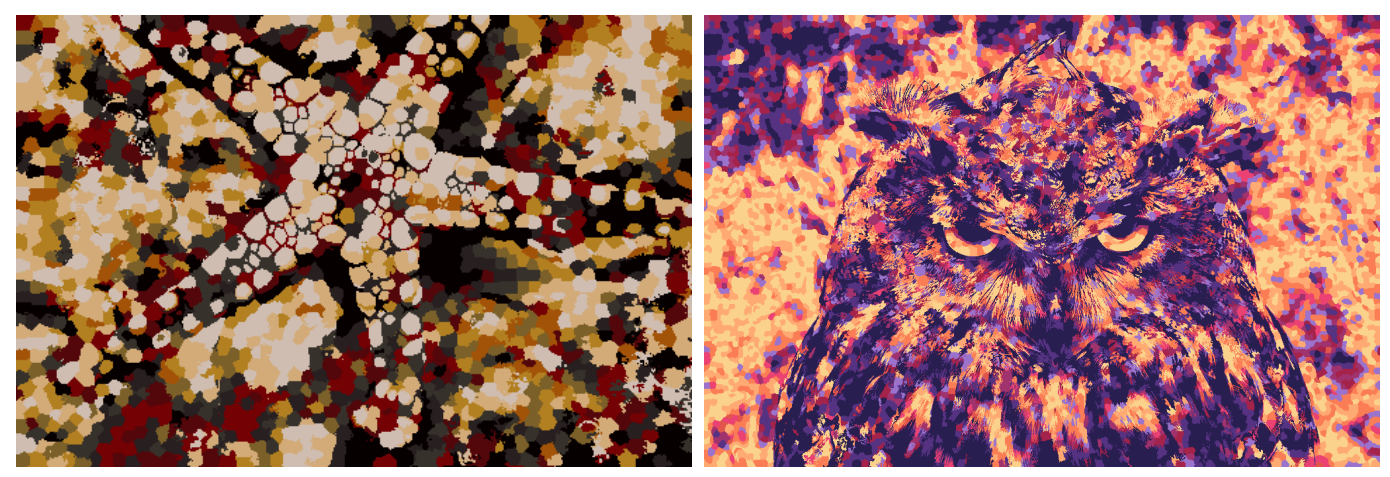

Figure 4.11: Recoloring with SLIC0 oversegmentation. 
the graph. The time complexity of single-source widest path is $\mathcal{O}(m+n \log n)$ for $m$ edges and $n$ vertices, using a heap-based priority queue in a Dijkstra search.

Table 4.1 shows the timing for creating the trees and color assignments of different images. For small images like the starfish, containing about $1.4 \mathrm{~K}$ regions, the recoloring algorithm takes about 0.007 seconds to construct the tree, while it takes about $0.3 \mathrm{~s}$ for larger images such as rust with $7.2 \mathrm{~K}$ regions. With a palette of 10 colors, the color assignments take $0.05 \mathrm{~s}$ and $1.2 \mathrm{~s}$ to recolor the starfish and rust respectively. The color assignment will take longer for larger palettes and images with a larger number of regions. We show the timing of tree creation and color assignments for all images in the gallery. The pre-processing steps of creating the graph adjacency list and histogram matching are also presented in the table.

\begin{tabular}{lccllc} 
Image & Tree creation & Color assignment & Graph & Total & \# Regions \\
\hline lanterns & $0.003 \mathrm{~s}$ & $0.02 \mathrm{~s}$ & $0.064 \mathrm{~s}$ & $0.087 \mathrm{~s}$ & $1 \mathrm{~K}$ \\
starfish & $0.007 \mathrm{~s}$ & $0.05 \mathrm{~s}$ & $0.07 \mathrm{~s}$ & $0.127 \mathrm{~s}$ & $1.4 \mathrm{~K}$ \\
lizard & $0.015 \mathrm{~s}$ & $0.08 \mathrm{~s}$ & $0.1 \mathrm{~s}$ & $0.195 \mathrm{~s}$ & $1.9 \mathrm{~K}$ \\
rust & $0.3 \mathrm{~s}$ & $1.2 \mathrm{~s}$ & $0.9 \mathrm{~s}$ & $2.4 \mathrm{~s}$ & $7.2 \mathrm{~K}$ \\
Venice & $0.3 \mathrm{~s}$ & $1.4 \mathrm{~s}$ & $0.9 \mathrm{~s}$ & $2.6 \mathrm{~s}$ & $7.7 \mathrm{~K}$
\end{tabular}

Table 4.1: Timing results for images with varying numbers of regions.

\subsubsection{Limitations}

Although in our experience our method works well for most combinations of image and palette, there are cases where the output is unappealing. When two similar regions are not neighbours, they may receive different colors; e.g., a sky area may be broken up by branches and different parts of the sky could be colored differently. Even adjacent regions may not receive similar colors if their average colors differ, introducing spurious edges into regions with slowly changing colors such as gradients or smooth surfaces. Out-of-focus backgrounds and faces are common examples producing such effects.

Figure 4.12 shows two failure examples. The woman's face is given an irregular, high-contrast color assignment, and her eyes look sunken. The busy background has 
similar contrast levels to the face, making the overall composition unappealing. In the Étretat results, large regions of different colors appear in the sky, which does not look attractive. Because the original regions have different average colors, our algorithm is likely to separate them regardless of the palette.

Our algorithm is at present strictly automated with no provision for direct user control beyond choice of palette and parameter settings. While these parameters provide considerable scope for generating variant recolorings, so that a user would have a wide range of results to choose from, direct control is not yet implemented. One might imagine annotating the image to enforce specific color selections or linking regions to ensure that their output colors are always the same. While it would be straightforward to add some control of this type, we have not yet implemented such features.
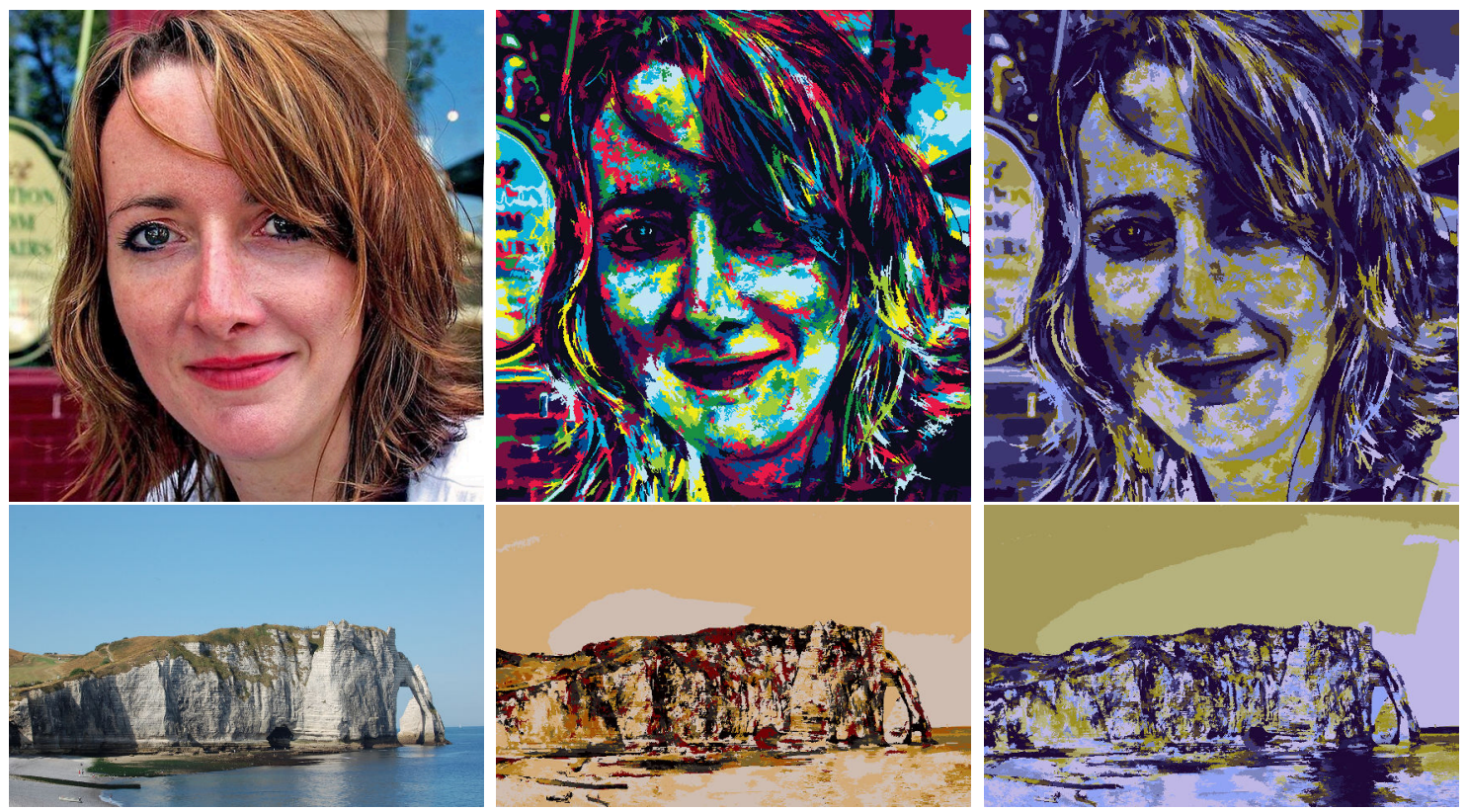

Figure 4.12: Failure cases.

\subsection{Variations}

In this section, we explore some variations of our region recoloring algorithm. We apply spatial blending to produce smooth color transitions. Then, we demonstrate that different color spaces and distance metrics can generate various results from one 
palette. Additionally, we present the recoloring results with a smaller number of color palettes.

\subsubsection{Blending}

In transferring the colors, we have intended to strictly preserve the palette and not add colors. However, we can also blend the colors, giving a more painterly style. Blending introduces new intermediate shades of colors. We suggest cross-filtering the recolored image with an edge-preserving filter such as CRGF [93]. This process smooths the areas away from edges while maintaining strong edges.

The cross-filtering mask size will affect the outcome. Larger masks will produce a stronger blending effect; small features will be smoothed out, and the output image will become blurry in regions lacking edges. Figure 4.13 illustrates examples of blending using masks of sizes $n=20,100$, and 300. Blending with $n=20$ only slightly modifies the image; for larger masks, the blending is more apparent. At $n$ $=300$ we can see a definite blurring in originally smooth areas, although blurring does not happen across original edges. Using a gray palette, can can obtain an effect resembling a charcoal drawing with larger masks.

\subsubsection{Color Spaces and Distance Metrics}

We can employ different functions for our color distance function $\mathcal{D}_{c}$. Different choices of color space and distance metric can affect the colorization results. Changing the distance metric will cause both the widest-path tree and the color assignment to change.

We have experimented with computing color distances with the Euclidean distance in RGB as well as using perceptually uniform measures CIE94, CIEDE2000, CIE76, and CMC colorimetric distances [114,134].

Both CIE94 and CIEDE2000 are defined in the Lch color space. However, CIE94 differences in lightness, chroma, and hue are calculated from Lab coordinates. CMC is quasimetric, designed based on the Lch color model. The CIE76 metric uses Euclidean distance in Lab space.

In Figure 4.14, we show different outcomes from different metrics using two palettes. We can observe the strong edge and contrast preservation, which is an apparent result of perceptual uniform metrics. More importantly, each metric gives 

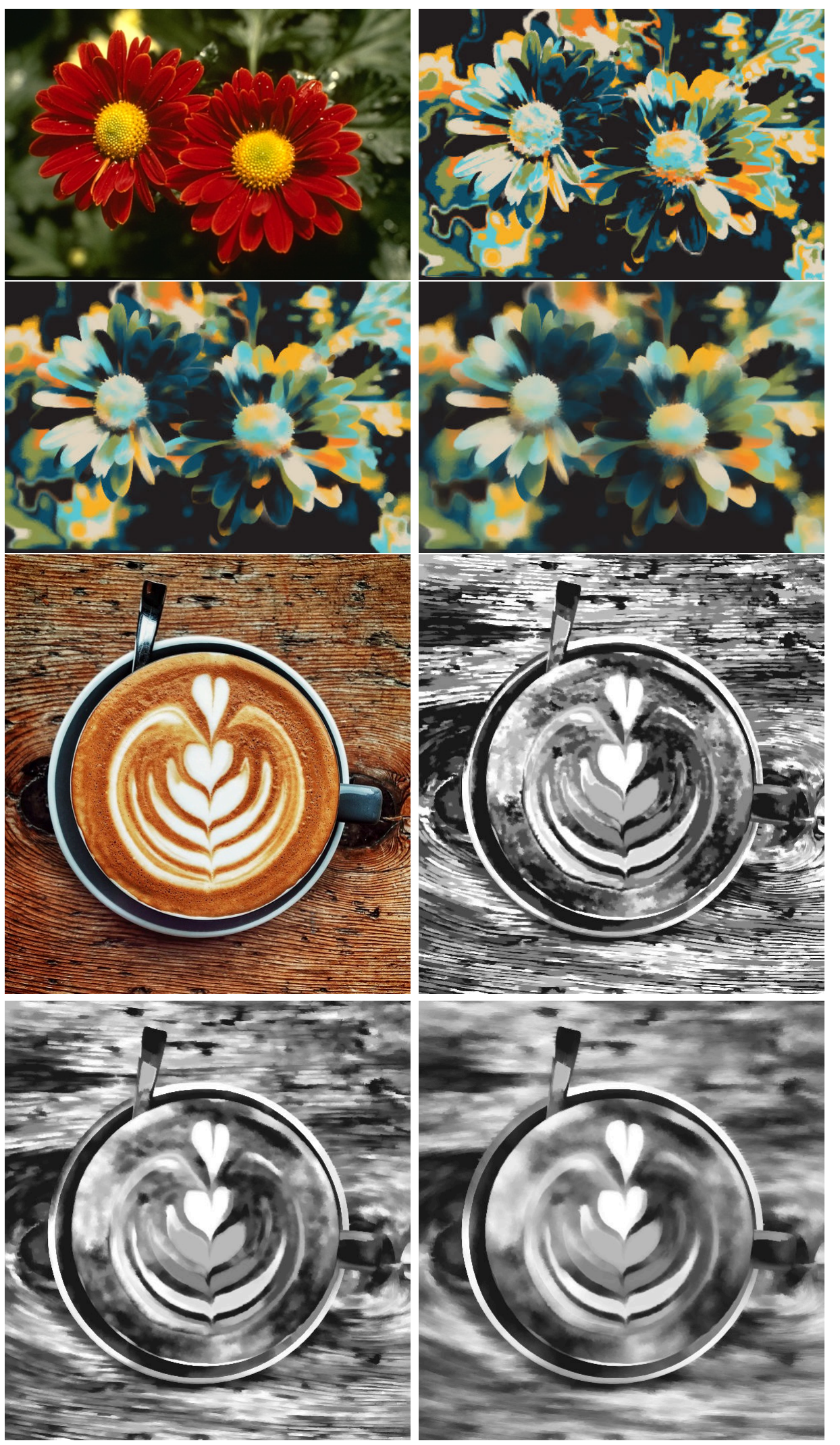

Figure 4.13: Postprocessing with an edge-aware filter. Left to right: original image; cross-filtered images with mask size 20, 100, and 300. 
a different variant, which allows a user to choose from different results. We can get interesting results from each metric. However, in our judgement, more attractive results are obtained from RGB and CMC colorspaces; the delicate features and image contrast are maintained, and objects are generally preserved. CIE94 and CIE2000 metrics are also effective, but we found that the CIE76 metric rarely creates interesting results.

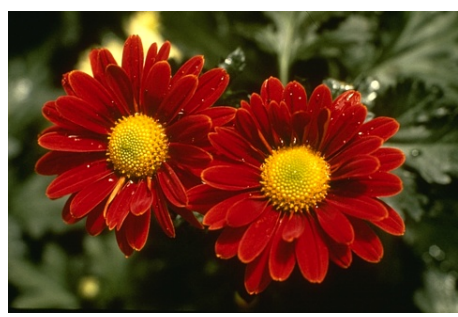

Input

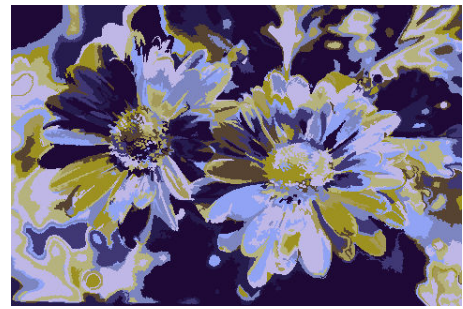

CIE94

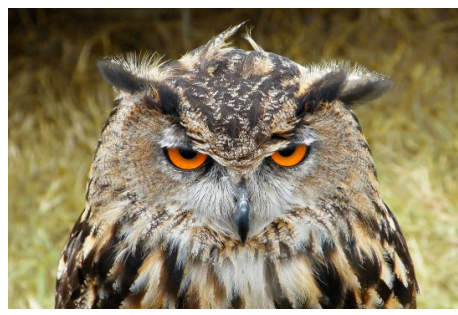

Input

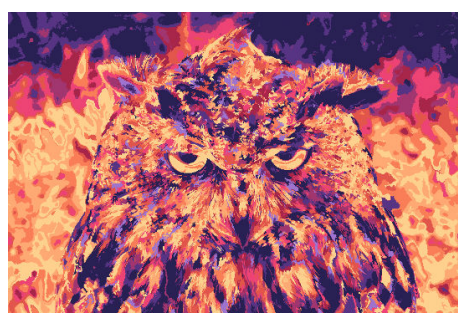

CIE94

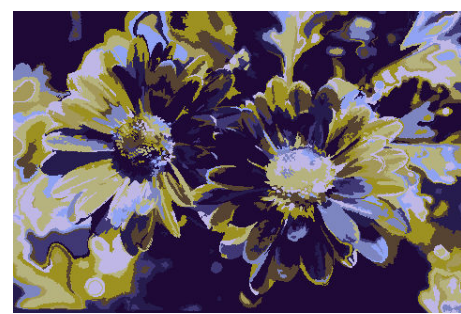

Euclidean

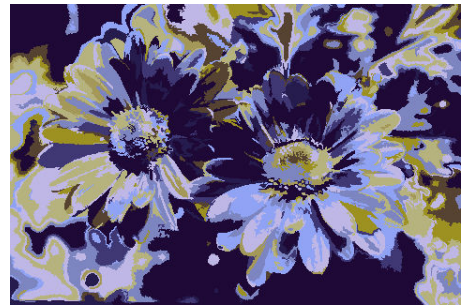

CIE2000

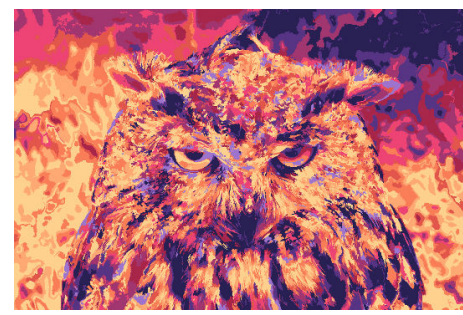

Euclidean

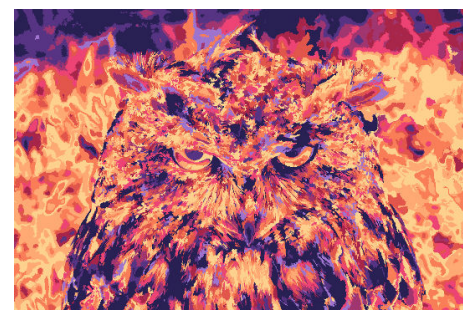

CIE2000

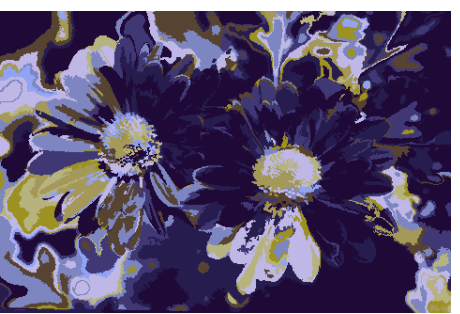

$\mathrm{CMC}$

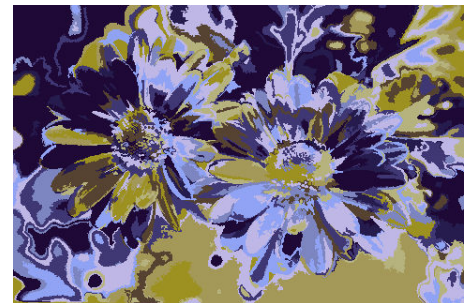

CIE76

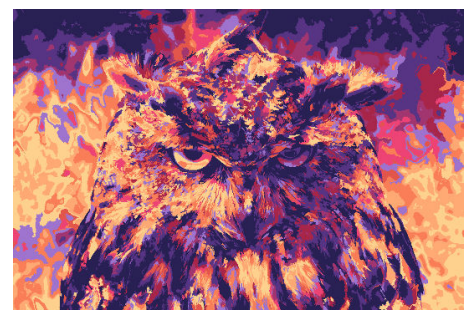

$\mathrm{CMC}$

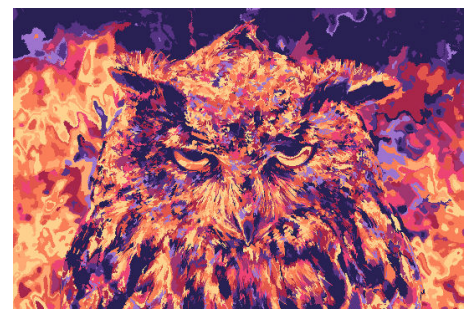

CIE76

Figure 4.14: Colorization with different distance measures. 


\subsubsection{Reducing Palette Size}

In designing our algorithm, we did not enforce constraints on the number of palette colors; however, we can reduce it to smaller numbers for stylization. Reducing the palette colors can affect how the textures will be perceived in the recolored image. A palette with a higher number of colors makes it possible to visualize more details of the image, and a palette with a limited number of colors simplifies the image contents.

In limiting the colors in a palette, we should keep those colors that can maintain the image contrast. Thus, for a two-color palette, we need two colors with different intensities. Eventually, the neighboring regions with small differences in their colors will be grouped in lighter or darker colors in the recoloring process.

Figure 4.15 illustrates recoloring with a palette of size 10, 8, 6, 4, and 2 colors. In reducing the palette colors, we discard those with the lowest contributions in recoloring with the palette containing them. From left to right, we can see a decrease of texture definitions on the starfish background, while the arms' structure, patterns on the starfish, and strong shadows are mostly preserved.
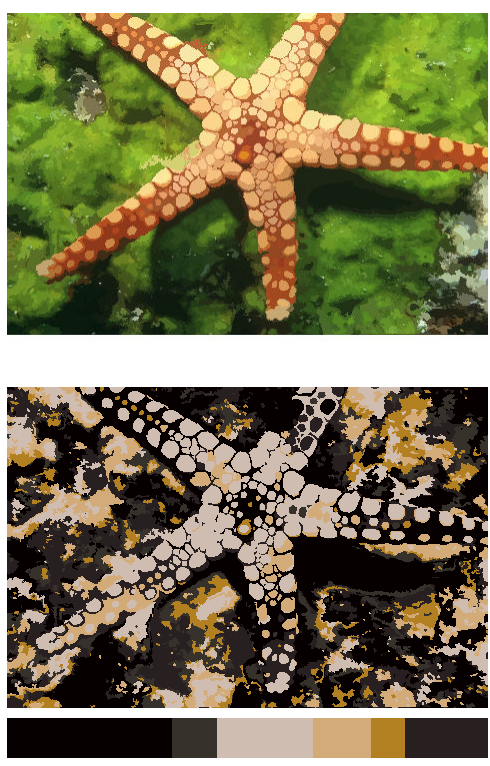
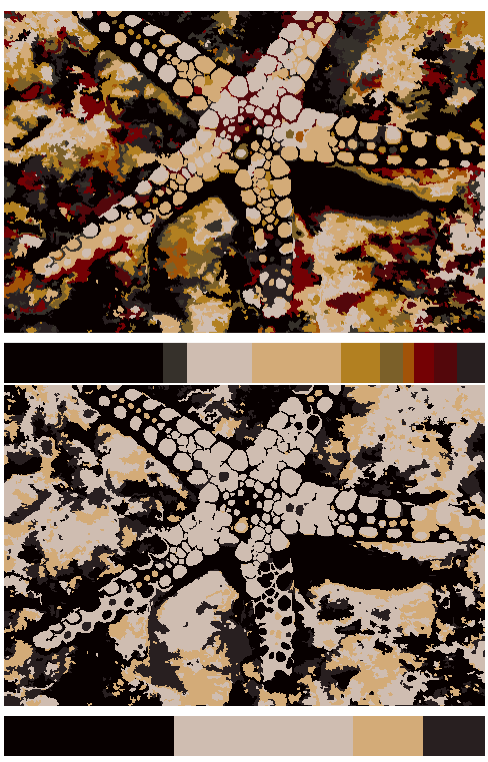
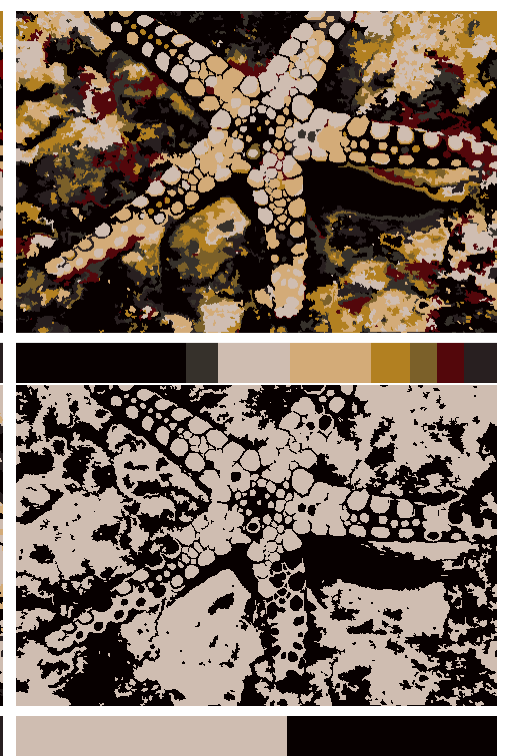

Figure 4.15: Reduced color palettes. Left to right, palette sizes are 10, 8, 6, 4, and 2.

In general, using a smaller number of colors simplifies the recolored image in some degrees. However, our algorithm is sensitive to color changes and tends to preserve them. By choosing a two-color palette, the algorithm reduced the distribution of color 
differences to the lowest capacity and simplified the image to some extend. However, it preserved some irregular textures, which might resemble a noise effect.

\subsection{Summary}

In this chapter, we presented a graph-based recoloring method that takes an oversegmented image and a palette as input, and then assigns colors to each region. The result uses the palette colors to portray the image content, but without attempting to match the input colors. Designing our algorithm with the widest path allowed us to maintain the image contrast and objects' recognizability. We demonstrated our results with different palettes. We achieved vivid recolorization effects, effective for most combinations of input images and palettes.

This work has been published as follows [10]:

- Rosa Azami and David Mould, "Artistic Recoloring of Image Oversegmentations," In Proc. Graphics Interface (2021). 


\section{Chapter 5}

\section{Improving Stippling in Extreme-tone Regions}

\section{$5.1 \quad$ Introduction}

Stippling uses small dots or circles to represent the image content. Stippling and other illustrative visualization techniques such as hatching have been widely used in artistic and scientific applications.

In computer graphics and non-photorealistic rendering (NPR), stippling techniques have focused on conveying local tone through the placement of a large number of dots. Many techniques have been developed for creating stipples where some of them had a focus on optimizing the point distribution and the point size $[31,32]$ or tone preservation $[23,78,116]$. Algorithmic stipple placement has tried to maintain some characteristics of handmade stippling images such as regularity and density of the stipples, yet various problems are not solved for stippling.

Stippling methods require enormous numbers of stipples to represent the black areas of an image. An image with extensive dark regions requires vast quantities of stipples and consumes excessive memory in storing them. We will cover each dark region with a polygon instead; doing so does not compromise the stippled appearance, since individual stipples in the dark regions were not visible anyway, and indeed the polygon can improve the image: the risk of spurious gaps is eliminated. Dark regions are determined by thresholding, and to eliminate the resulting discontinuities, we employ a Poisson solver to reconstruct a smooth image.

Light areas pose a different problem: the few stipples placed there distract rather than inform the viewer, and as noted by Deussen and Isenberg [33], removing stipples 
placed on white areas produces an image similar to what artists create. This observation had been informally used earlier, where authors such as Secord [116] thresholded away their light background. We apply thresholding, and also supply a postprocessing method that systematically removes additional stipples from light areas when they seem not to contribute to image structure.

We apply our method in conjunction with two existing stippling algorithms: structure-preserving stippling [78] and a recent Voronoi-based stippling method called Linde-Buzo-Gray stippling [31]. The resulting images show marked visual improvement despite the use of fewer stipples. Our main contributions are as follows:

- Illustrating dark regions: We identify the dark regions of the image and cover them with solid polygons instead of stipples. To eliminate discontinuities, we smoothly reconstruct the image in a small area surrounding each dark region.

- Controlling stipple placement in light regions: We eliminate unnecessary stipples on light regions, both through preprocessing to prevent stipple placement in very light areas and through postprocessing to identify and remove such stipples.

Further, we suggest a method to preprocess the image so as to better show small details, measuring local contrast and increasing it for selected elements. Our approach is not necessarily suitable for all images, but shows some improvement in specific instances.

\subsection{Method}

This chapter addresses two main issues. First, traditional stippling methods match the tone of dark areas by filling them with huge numbers of stipples; we propose instead covering each dark area with a polygon. Doing this in a naïve fashion would produce visible discontinuities; we reconstruct a small region surrounding each dark region so as to produce a smooth transition. Second, tone-based stippling can produce isolated stipples in light regions; such stipples are distracting and may not represent any specific image content. We propose a postprocessing step that identifies isolated stipples and removes them from the image. The stages of our algorithm are illustrated in Figure 5.1. 


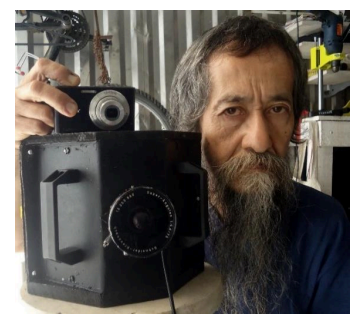

a) Image $I$

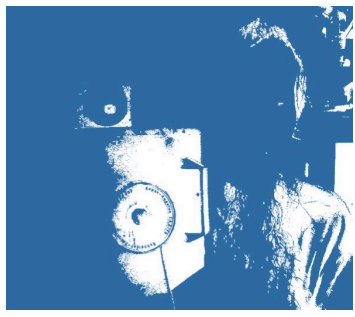

b) Dark regions

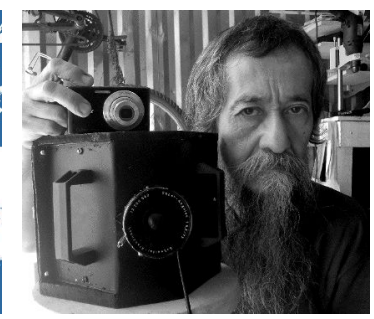

c) Reconstructed image

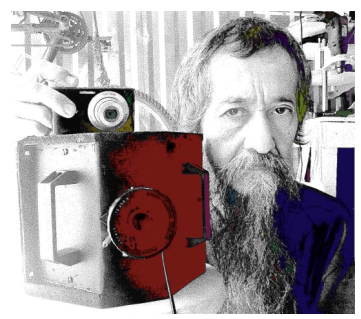

d) Stippling and polygons

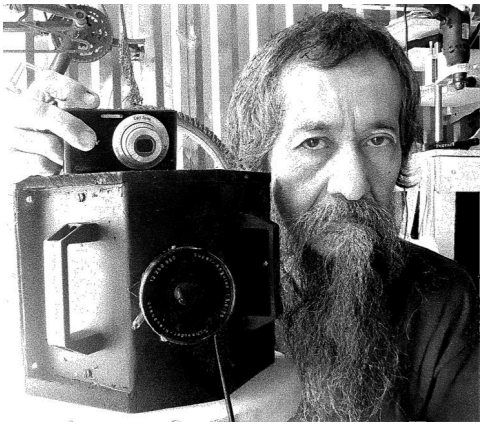

e) Final result

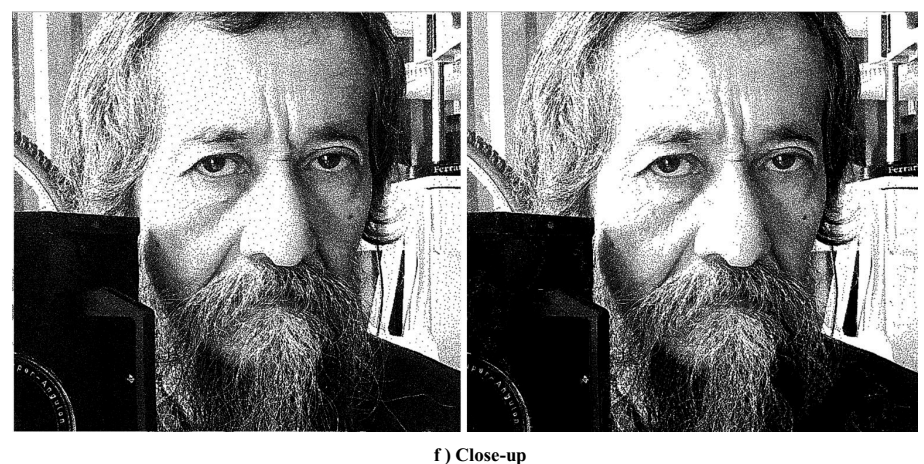

Figure 5.1: Steps of our method: $a$ ) original image; $b$ ) threshold mask; $c$ ) reconstructed image after Poisson blending; $d$ ) results from filling polygons; $e$ ) final result after stipple removal; $f$ ) close-ups of original stippling and final result of the polygon filling followed by stipple removal steps.

We perform two thresholding operations to identify dark and light regions. We will represent dark regions with polygons rather than filling them with numerous stipples. In light regions, we set the image's intensity to maximum in order to prevent any stipples from being placed there; in such areas, stipples would in any case have been infrequent and unrelated to image content, making them appear spurious to a viewer. Figure $5.1(a)$ shows an input image and $(b)$ shows the dark regions obtained by thresholding. In all cases, when thresholding yields a connected component that is too small (in practice, with an area smaller than 20 pixels), we ignore this region and use the original image values in subsequent stippling. Larger regions are processed as follows.

At cutoff thresholds, the transitions between the black (or white) regions and the nearby areas are discontinuous. In order to obtain smooth transitions, we reconstruct a narrow boundary zone by solving a Poisson equation 2.1. When the reconstructed image is passed to the stippling algorithm, the thresholded pixels are labeled as "done", thus preventing stipples from being placed there. Figure $5.1(c)$ illustrates the reconstructed gray image and $(d)$ the result of applying stippling to the reconstructed 
image. Note that, we just showed the mask of the dark regions in this figure.

Once the stippling is complete, we remove additional potentially spurious stipples appearing everywhere in the image. We remove stipples through two post-processing steps. In the first step, we remove the most isolated stipples: those that have at most one other stipple nearby. In the second step, we remove additional stipples deemed not to contribute to the image content, identified as follows. We compare two quantities: pixel intensity of the target stipple, and the average intensity of the original image in a range surrounding the stipple location. If the difference between the quantities is large we keep the stipple. If the difference between the quantities is less than a threshold, we do further analysis, basing our decision on the number of stipples nearby: when there are more than five stipples within a given distance, the stipple is considered to be part of the image structure and hence kept, whereas if there are fewer, the stipple is removed. Figure $5.1(e)$ illustrates the final result after stipple removal and $(f)$ shows a close-up of the original stippling on the left and our final result of the polygon filling and stipple removal steps.

Tone-preserving stippling can straightforwardly show image details in darker regions of an image: there are plenty of stipples, and small variations in their distribution are visible. In lighter regions, however, showing details is more difficult. The requirement of tone preservation enforces a low stipple count. The cat's white whiskers against a bright background, for example, may no longer be visible after stippling. Although this is a natural outcome of tone-preserving stippling, we would still like to show these details. We propose an optional preprocessing step to identify and amplify these features in the input image, darkening light details when doing so increases the local contrast. Details of this process are given in Section 5.2.3.

\subsubsection{Illustrating Dark Regions}

We will avoid placing stipples in dark regions and instead cover each region with a polygon. First, we threshold all pixels $I(x) \leq T_{\text {low }}$ to 0 . We replace these thresholded regions with solid polygons. To avoid discontinuities between the thresholded regions and their surroundings, we reconstruct a transition region between the thresholded pixels and the unaltered image regions. For reconstruction, we consider an upper threshold $T_{\text {high }}$; we can then create a transition region $\Omega$, which we define as the set of pixels such that $T_{\text {low }}<I(x) \leq T_{\text {high }}$. 
We reconstruct all transition regions by solving a Poisson equation over the image [107]:

$$
\Delta I=\operatorname{div} \mathbf{G},
$$

with boundary conditions given by the pixels adjacent to the region; note that the pixel values are taken post-threshold. The Poisson equation uses the image gradient vector field $\mathbf{G}$. We stretch $\mathbf{G}$ so as to account for the greater range of intensities the transition region needs to cover: $\mathbf{G}$ is scaled by $(a+b) / b$, where $a=T_{\text {low }}$ and $b=T_{\text {high }}-T_{\text {low }}$. Figure 5.2 shows the pixel intensities near a dark region before and after the reconstruction; the discontinuity produced by thresholding has been eliminated by the reconstruction process. On the figure's right, we can see the effect on a sample image region. Thresholding without reconstruction of transition regions using equation 5.1 produces a visible polygon boundary. With reconstruction, no distinct boundary is visible: the stipple density gradually eases as we move away from the polygon. Note that the same polygon is used in both cases. With reconstruction, the fringe around the polygon is darker; this is not usually noticeable without close comparison with the original, but increases the number of stipples in the output, slightly offsetting the gains from eliminating the stipples in the polygon.
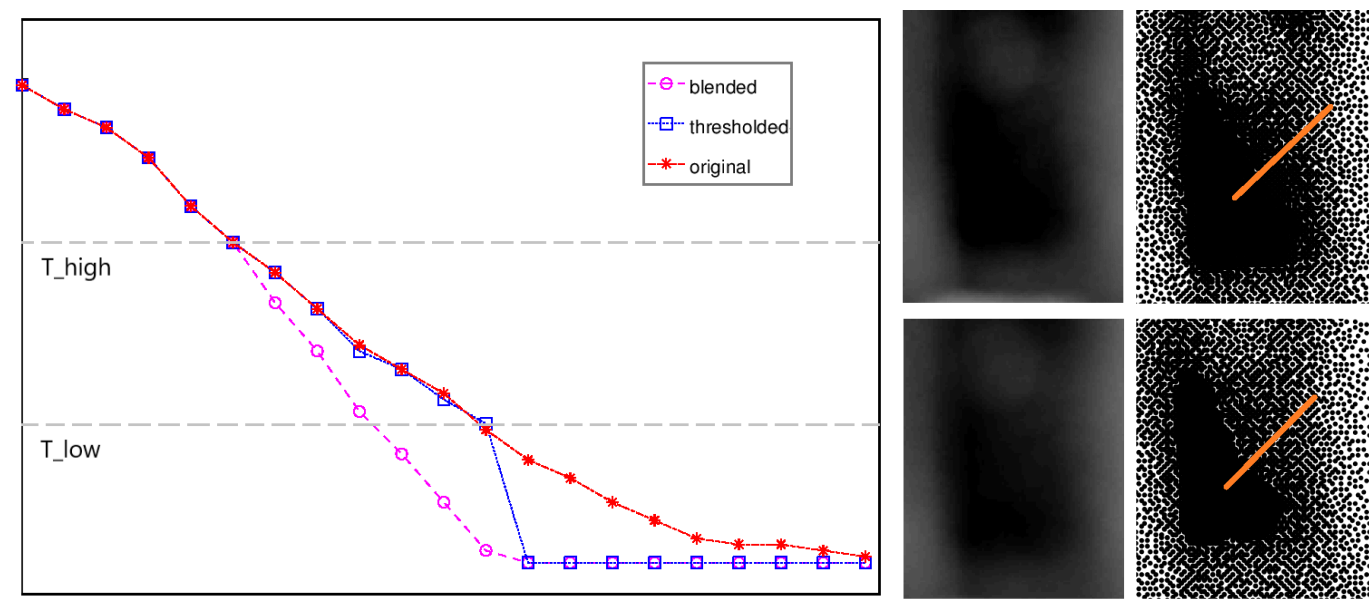

Figure 5.2: Transition from a dark region to its surroundings. The graph shows the original intensity profile (star), the thresholded values (square), and the results of blending (circle). On the right, the top images show the grayscale image and corresponding smooth transition of stipples and the bottom images are the results of hard thresholding.

To obtain a polygon approximating a region, we find the pixels making up the 
exterior contours and treat each as a vertex; the sequence of vertices is the polygon. Figure 5.3 shows the thresholded regions followed by the resulting stippled image in which the dark regions are shown with solid black polygons.

The blended grayscale image is the input to the stippling algorithm. The pixels in thresholded regions are labeled as "done" so that the stippling algorithm will not place any stipples in these regions. The smooth gradient arising from the Poisson solution guarantees a continuous intensity transition from the thresholded areas towards their surroundings.

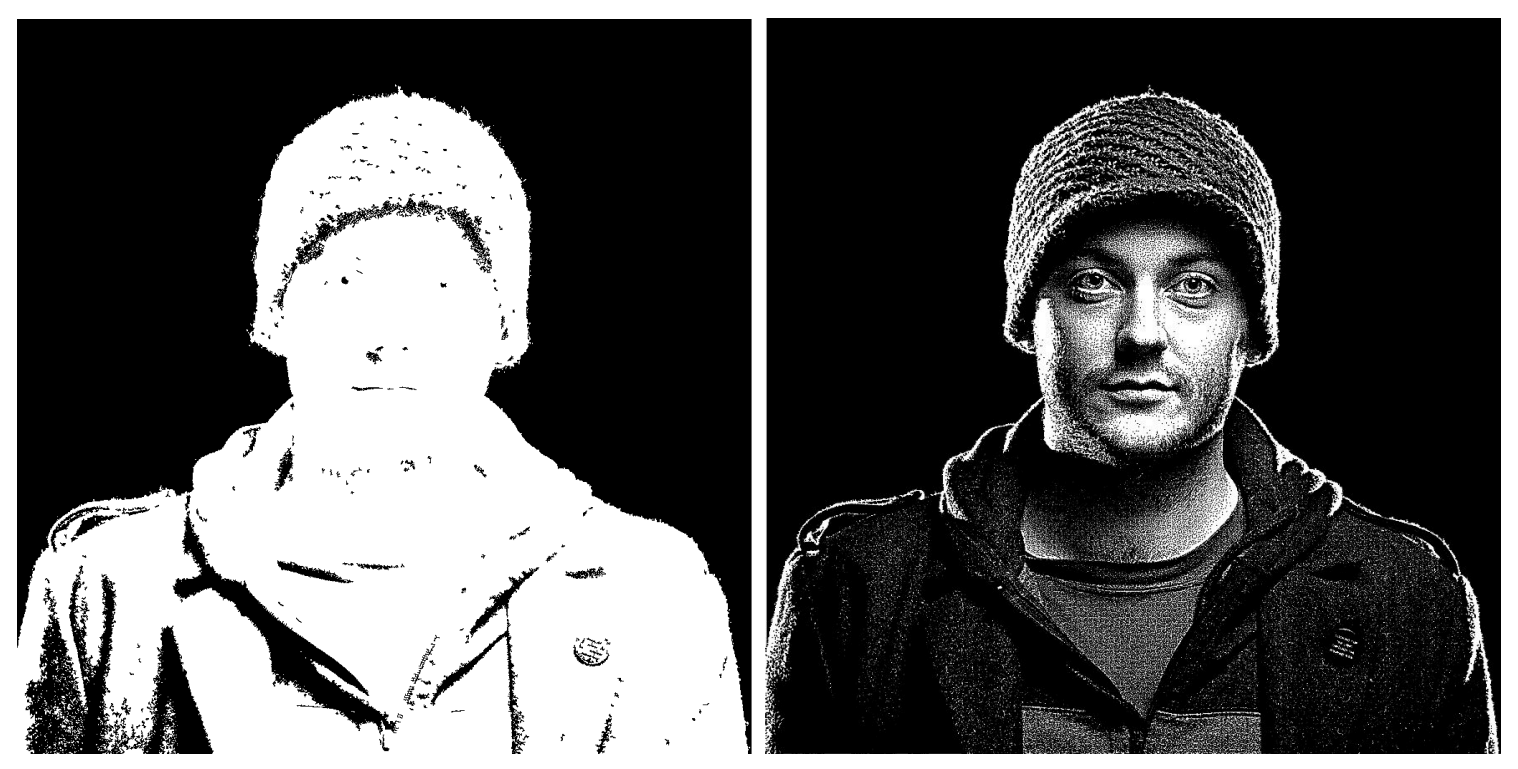

Figure 5.3: Left: thresholded regions; right: stippled image with dark regions covered by polygons.

Akin to our treatment of the the dark areas, we also threshold the bright image regions. The effect here will be to prevent stipple placement in areas that are close to white, since they will now be thresholded to maximum brightness. Note that the light and dark thresholding and reconstruction can be done separately, since their transition regions, consisting of pixels near in intensity to the thresholds, can never overlap.

\subsubsection{Stipple Removal in Light Regions}

Thresholding prevented putting stipples in very light regions. However, treatment of medium-intensity regions needs another step. With thresholding, we can remove 
many unnecessary stipples, but it is not possible to set a threshold value that can remove all the isolated stipples and yet keep the stipples that contribute to the image content. A single stipple can not express any features and the human eye can not interpret it as anything meaningful; with tone-preserving stippling, stipples in medium-intensity images will be spread out and many can be eliminated. We use a conservative threshold (e.g., $T_{h}=235$ or 245 ) to prevent eliminating any potential structure, and apply post-processing steps to identify and remove remaining isolated stipples.

Figure 5.4 illustrates examples of unnecessary stipples and the effect of their removal from the images. We remove such stipples in two phases. First, we discard the stipples that have at most one other stipple within a radius $r$; these low-density, non-clustered stipples do not indicate any image detail. We run the first step over all stipples. Then, we apply a second round of stipple removal, only considering stipples whose pixel intensities exceed a threshold, say $L$; we use $L=128$ in practice.
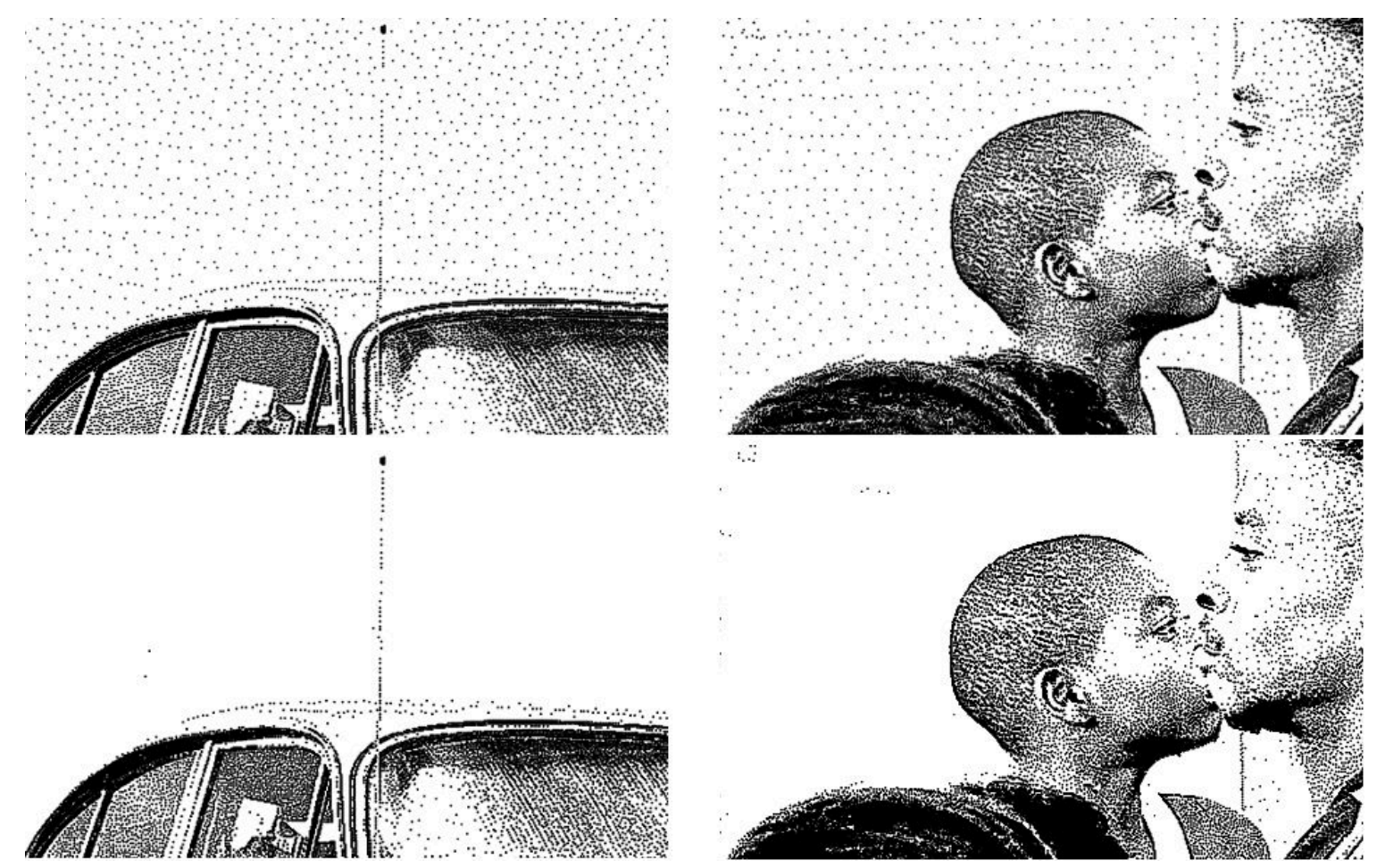

Figure 5.4: Selected details of the car and New Jersey images. On the top row, note the unnecessary stipples in the sky above the car and in the background behind the kid. On the bottom row, the unwanted stipples are removed from the background. 
Our removal test involves checking two quantities: pixel intensity at the target stipple, and the average intensity of the original image in a range surrounding the stipple location. The intensity of the pixel where the target stipple is centred is given by $I_{t}$ and the average intensity of the stipple's neighborhood is $I_{n}$. Let $q=\left|I_{n}-I_{t}\right|$. We decide whether or not to remove the target stipple based on the relationship between $q$ and the threshold $T_{a}$ :

a) If $q \geq T_{a}$, we keep the stipple.

b) If $q<T_{a}$, we decide instead based on the number of nearby stipples. The stipple will be removed when the number of nearby stipples is less than some constant c.

We used the threshold $T_{a}=25$. We set the search radius $r=10$ and the stipple count threshold $c=5$. Parameters $c$ and $r$ together yield a measure of local stipple density and should not be adjusted independently, but note that we cannot simply merge them since we require a parameter for the region size over which count (or density) should be evaluated. An example of the stipple removal process is given in Figure 5.5.

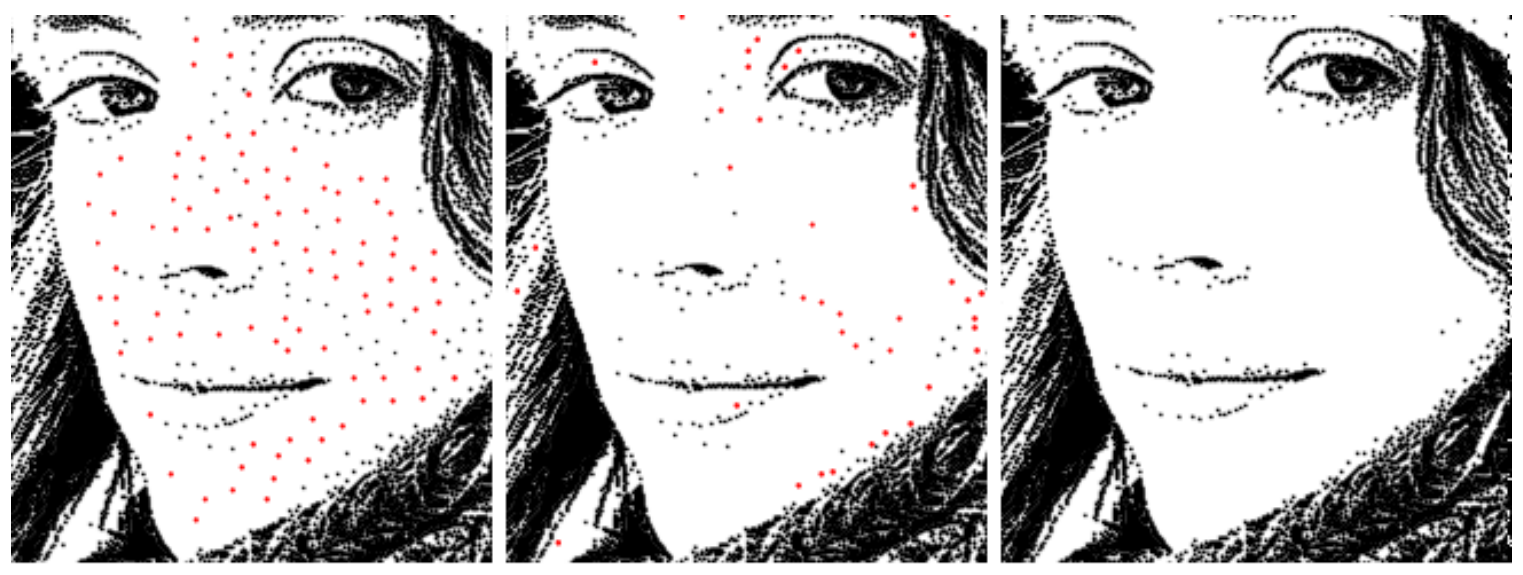

Figure 5.5: Stipple removal process. From left to right: result before stipple removal; stipples with 0 or 1 neighbours removed; final result after removing stipples with fewer than $c$ neighbours. 


\subsubsection{Detail Enhancement}

Low-contrast features in bright regions of an image are lost under stippling with faithful tone reproduction: there will simply not be enough stipples to indicate any contrast. We suggest judiciously darkening such features in a preprocessing stage such that the subsequent stippling method will place stipples there.

We apply an edge-preserving filtering algorithm [94] and compute the residual. Regions where the residual is positive correspond to features brighter than their surroundings. Such regions are candidates for darkening; to darken an area, we will add its reversed residual to the original image, akin to unsharp masking. Note that only regions with positive residuals are considered, so that only darkening can occur, not lightening.

We cannot darken all positive-residual regions indiscriminately, however. In textured areas, for example, darkening the bright parts and leaving the rest alone will ruin the image detail. Instead, we test whether reversing the residual will increase or decrease contrast; if contrast is increased with reversal, the reversal is beneficial and we accept it.

The test operates as follows. We calculate two average RMS contrasts [106] for each group of connected components within a neighbourhood: one with the original values and the other with positive residuals reversed. If the original contrast is greater, we do nothing, otherwise we reverse the positive residuals in that area.

Figure 5.6 shows an example image and the corresponding positive residuals. When using this process, some windows of the building are made clearer. The drawback is that the original image is no longer precisely reproduced.

\subsection{Results and Discussion}

In this section we show results from our method and discuss the advantages and limitations of the approach. Most results from SPS [78] use the following parameters: $G_{+}=G_{-}=5$; mask with radius $D / 2=10$. For most images, we set $T_{\text {low }}=10$ and $T_{\text {high }}=50$, with a symmetric set of thresholds to deal with light regions, an upper threshold of 245 and a lower threshold of 205. We used different thresholds for the parrot and headlight images, where $T_{\text {low }}=20$ and the threshold for bright areas was 235. In general, thresholding operations are sensitive to the choice of threshold, so 

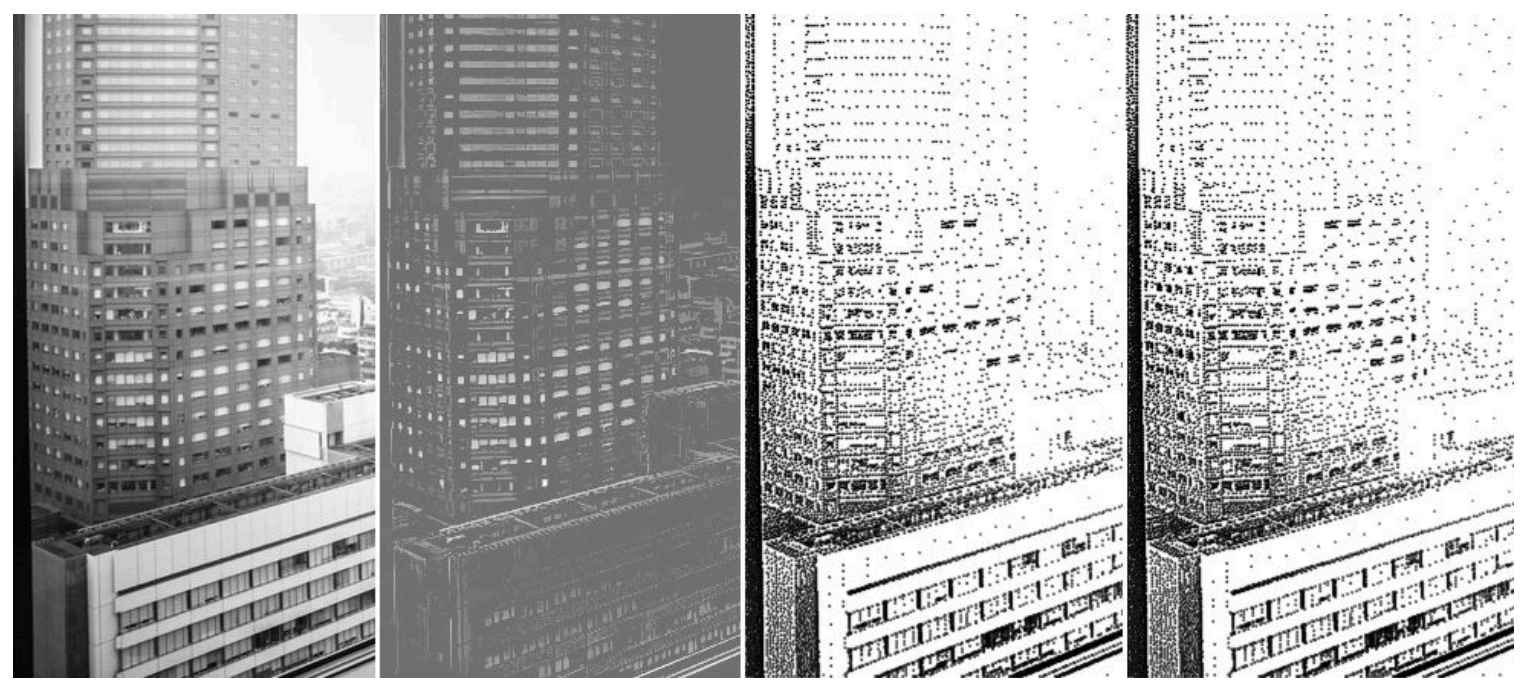

Figure 5.6: Effect of detail enhancement. From left to right: original gray image; positive residuals; original structure-aware stippling; stippling after detail enhancement.

the user may wish to select different values for a particular input image. We also show the effect of our method on LBG stippling. We set the minimum and maximum stipple size to 2 and 8 , respectively, to get comparable contrast to the input image. In the following section, we first discuss some results from structure-preserving stippling, then a few results from LBG stippling.

\subsubsection{Visual Evaluation}

Structure-preserving stippling. Our main contribution involved representing the darkest areas of an image with solid polygons rather than filling them with stipples. We use polygons for the dark background behind the girl in toque in Figure 5.7, black stripes in headlight and the dark woods behind parrot; these results are shown in Figure 5.8. The visual effect is quite good: the polygons are not intrusive and can occasionally be improvements over the stippled black areas, e.g., in the rare case where an inopportune arrangement of stipples leaves a tiny gap. The transition from the polygon exterior to the rest of the image appears natural, preserving the surrounding detail and edge placements. The top detail panel in the first row of Figure 5.7 shows sample transitions.

Stipples have been eliminated from the brightest regions of the image through a 


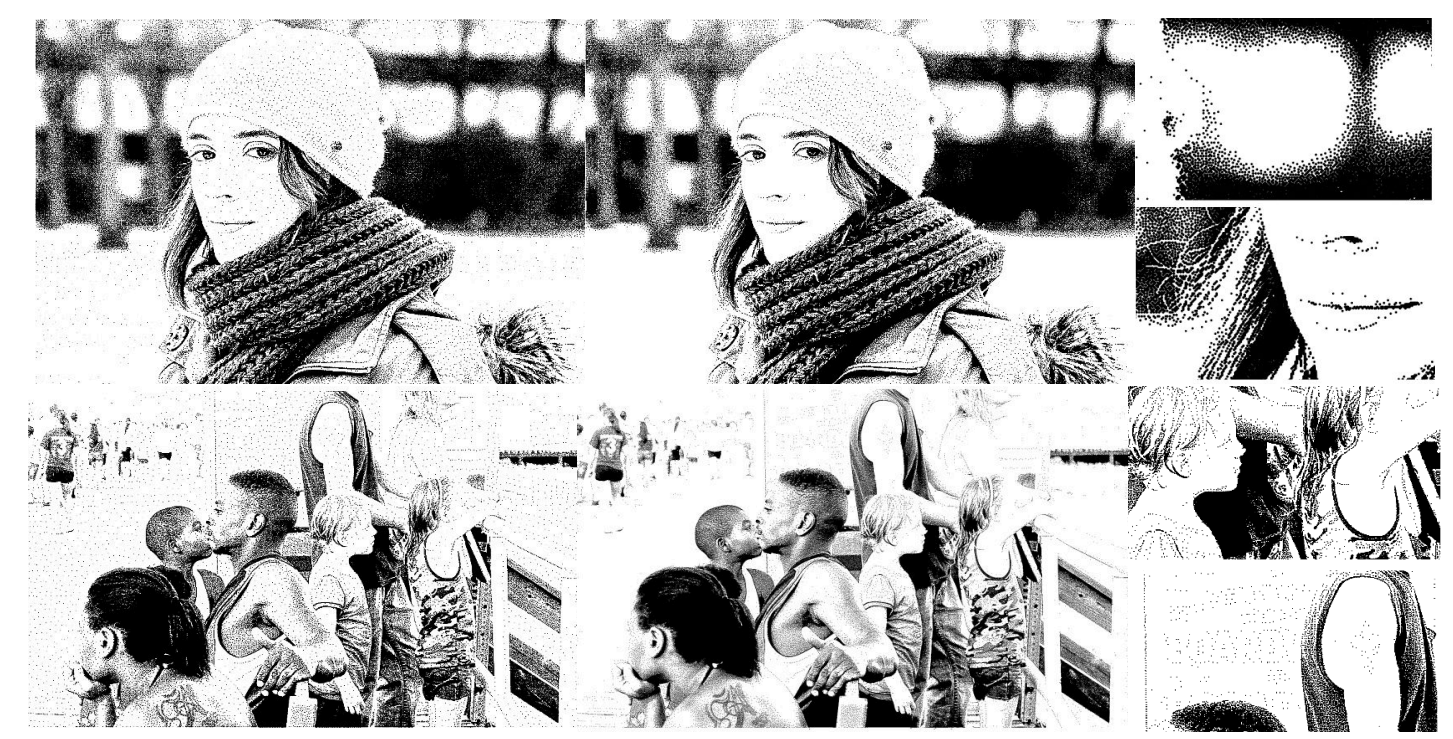

Figure 5.7: Smooth transitions and pattern preservation. Left column: original stipplings; middle: our results; right: details, in which we see smooth transitions from dark to light regions and preservation of facial features in toque, and the kid's face and tattoo in New Jersey.

similar thresholding process. Few stipples existed in such regions in the first place, and the transition away from such regions also exhibits few stipples, so the effect is not very dramatic. Nonetheless, removing these few stipples can improve the image; the sky seen through the big window of the city image in Figure 5.9 provides an example, indicated by the blue circle in the middle. Removing distracting stipples in light areas also reduced the number of stipples; however, more importantly, it improves the clarity of the image. The girl's face in toque has been largely cleared, eliminating distractions. The stipples that had been sparsely distributed over the top of the headlight represented neither gradients nor structure, so removing them made the image clearer.

Our method can reveal patterns and preserve some thin structures that were hidden under the original stippling. For example, in Figure 5.7, elements in the New Jersey image have become more prominent, including the kid's face, the tattoo on the man's arm, and the text on the window.

We partially preserved low-intensity but structural features by reversing residuals. In Figure 5.9, some of the small windows on the high building of the city and in cat the whiskers on the left and some details in the fur have been darkened; these 

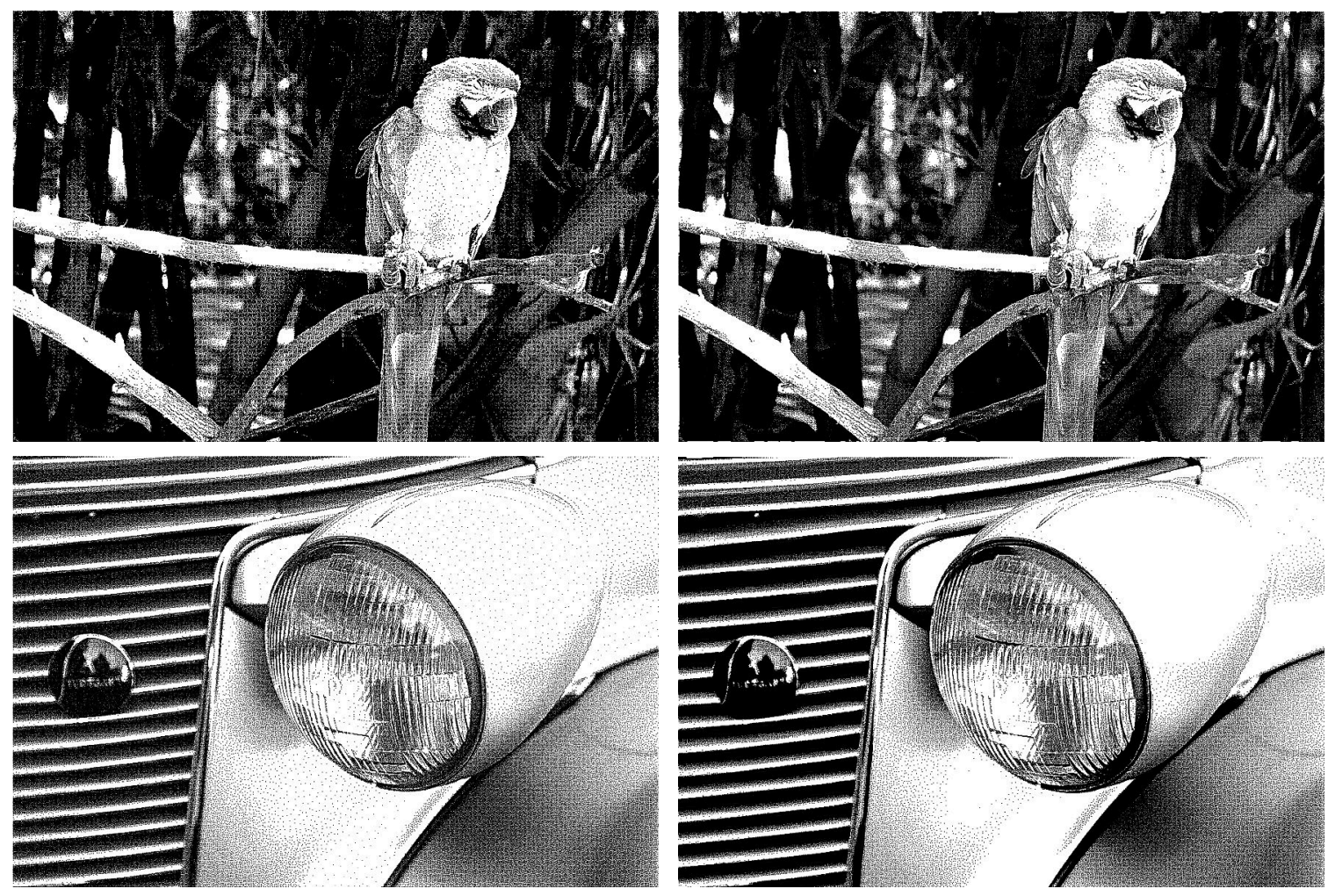

Figure 5.8: Results after applying our method on parrot and headlight based on structure-preserving stippling [78]. Left: original stipplings; right: our results.

were light details in light regions and would not normally be feasible to show with dark stipples. The two circles indicate some places where differences between the original and enhanced images appear; the reader may wish to zoom in for a better comparison. Note that the whiskers on the right were not darkened since doing so would have reduced the contrast of the region. Similarly, not all windows of the city were darkened.

Linde-Buzo-Gray stippling. Figure 5.10 shows LBG stippling before and after applying our algorithm. The black polygons cover the dark chair backs in theater and water in the cave in the Oparara image. Filling the gaps between stipples in the dark regions has increased the image contrast and improved the appearance. We also removed spurious stipples from the theater screen. Intermediate regions like the carpet remained unchanged. 

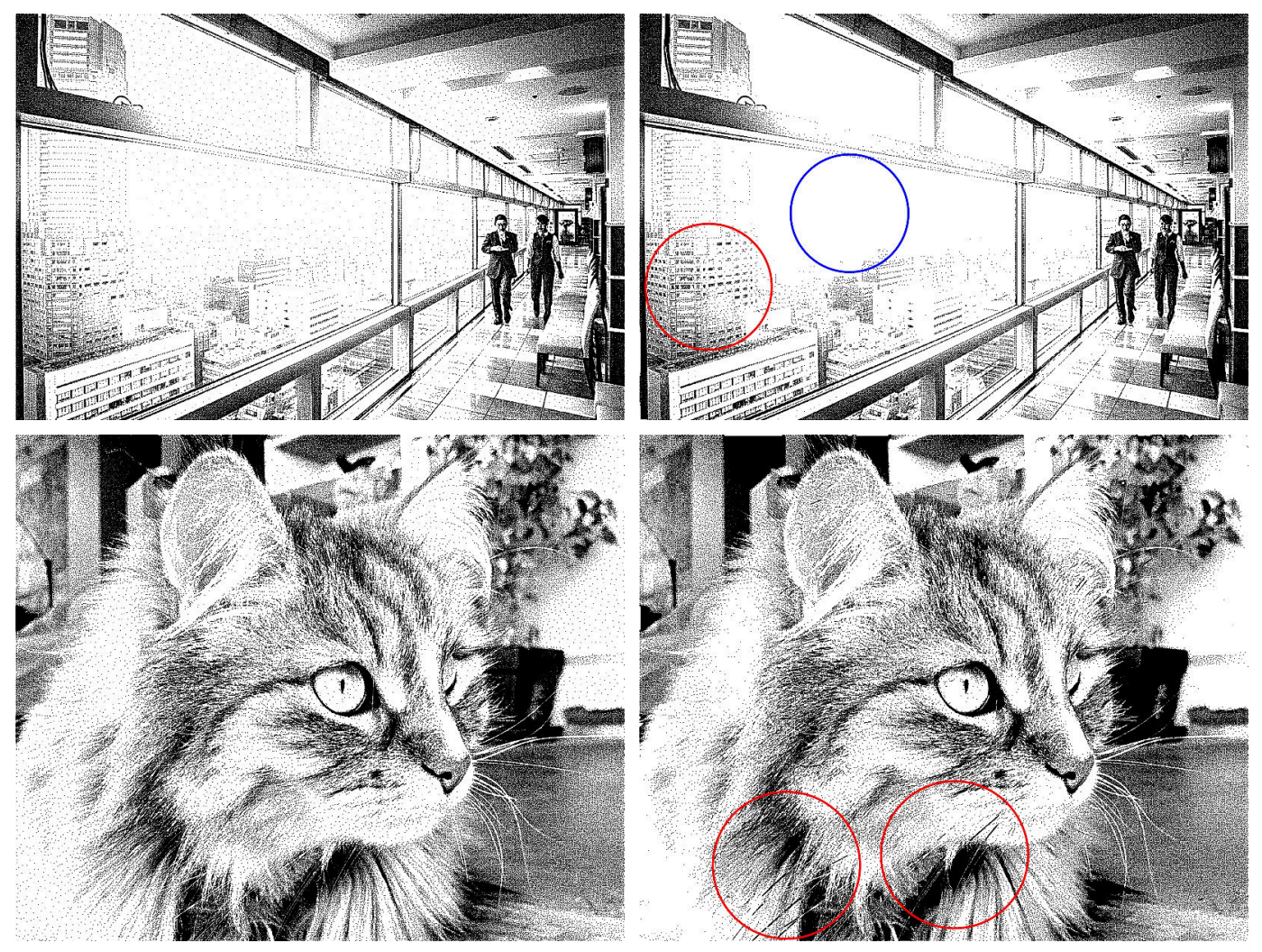

Figure 5.9: Results of detail enhancement. Left: original SPS; right: detail enhancement. Red circles show particular cases of detail enhancement and the blue circle shows stipple removal. Zoom in to see details.

\subsubsection{Performance}

Tables 5.1 and 5.2 show the number of stipples for selected images before and after applying our algorithm on the SPS [78] and LBG [31] methods, respectively. Images with large dark areas experienced significant reductions in stipple count. For example, the old man image generated almost 82k stipples under the original structure-preserving stippling method; this number decreased to $26 \mathrm{k}$ after applying our stipple removal method. The tables also show the number of polygons; a small number of polygons were added relative to the number of stipples eliminated.

All our results were generated on a laptop with an Intel Core i7-4510U CPU 2.0 GHz. Reconstructing the blended gray image takes roughly $0.9 \mathrm{sec}$ for an image of size $1600 \times 1200$ pixels. Stippling process takes almost one second longer with the stipple removal steps to produce $107 \mathrm{~K}$ stipples. Timing directly depends on the size of the image and the number of stipples. Including our method in the stippling 

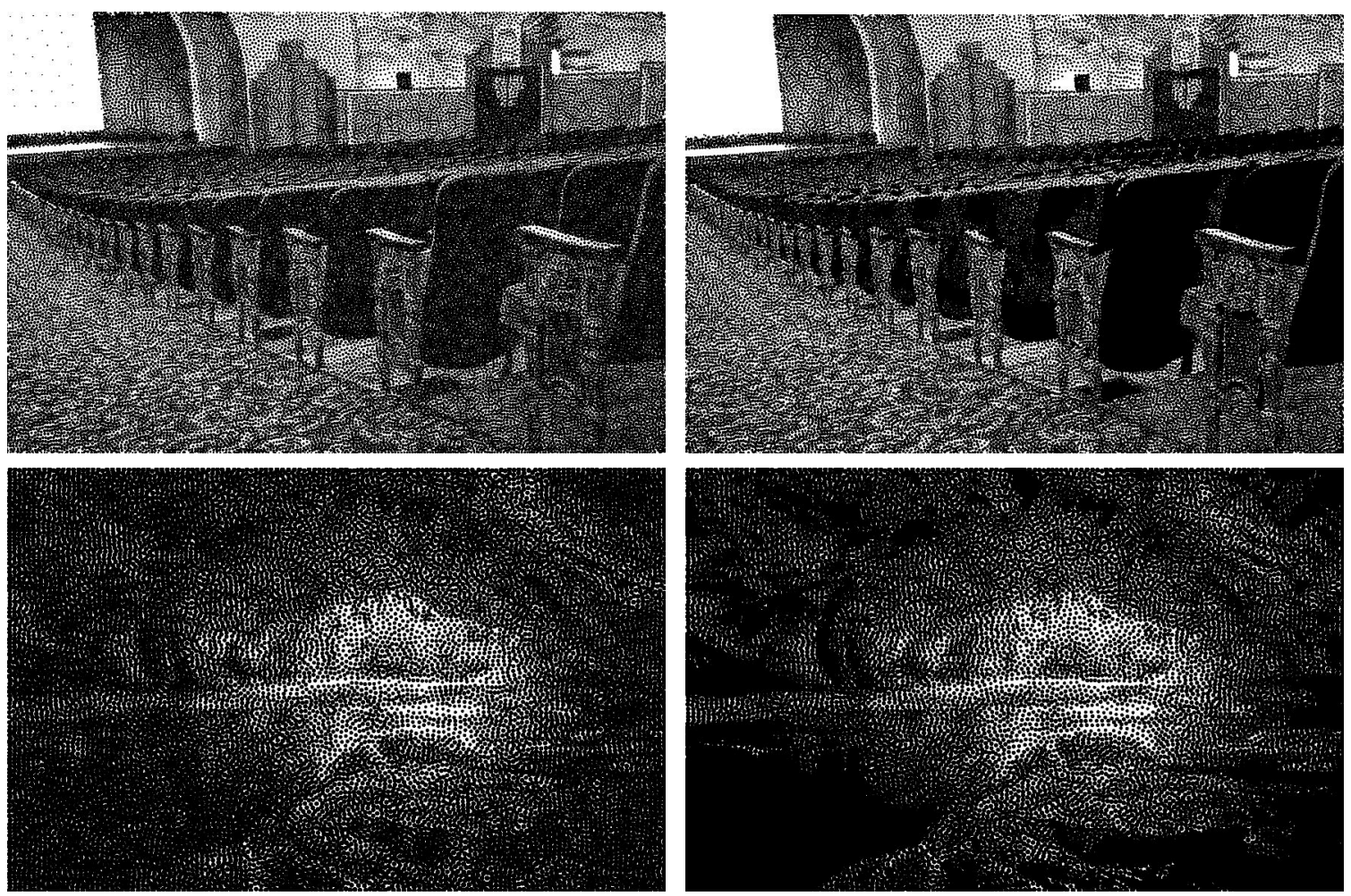

Figure 5.10: LBG stippling with our algorithm on Theater and Oparara images. Left column: original LBG stippling and right column: LBG stippling after applying our method.

creation increases the overall time by no more than a few seconds, a small fraction of the overall cost for most stippling methods. We see a significant reduction in size of the vector graphics files of many images; for example, the scalable vector graphics file for the old man decreased from 3.6MB to 1.2MB based on SPS method and the toque file size went from $6.25 \mathrm{MB}$ to $4.9 \mathrm{MB}$. However, the darkened fringe around black polygons requires more stipples than the same region in the original image; for sufficiently small polygons, this effect outweighs the savings from eliminating stipples within the polygon, and in rare cases the overall stipple count for the image increases.

\subsubsection{Limitations}

Our method usually improves image quality, according to our subjective assessment. Nonetheless, it suffers from some limitations, generally arising from the use of solid colors (whether black or white) to represent large image regions. By thresholding the 


\begin{tabular}{lrrrr} 
Image & \# Stipples (SPS) & \# Stipples (ours) & Reduction & \# Polygons \\
\hline Toque & $139 \mathrm{~K}$ & $107 \mathrm{~K}$ & $23 \%$ & 121 \\
Headlight & $148 \mathrm{~K}$ & $125 \mathrm{~K}$ & $15 \%$ & 32 \\
Old man & $82 \mathrm{~K}$ & $26 \mathrm{~K}$ & $67 \%$ & 22 \\
Oparara & $293 \mathrm{~K}$ & $206 \mathrm{~K}$ & $30 \%$ & 140 \\
Theater & $265 \mathrm{~K}$ & $255 \mathrm{~K}$ & $4 \%$ & 96
\end{tabular}

Table 5.1: Stipple and polygon counts from SPS before and after applying our method.

\begin{tabular}{lrrrr} 
Image & \# Stipples (LBGS) & \# Stipples (ours) & Reduction & \# Polygons \\
\hline Toque & $10.6 \mathrm{~K}$ & $8.8 \mathrm{~K}$ & $17 \%$ & 121 \\
Headlight & $13.26 \mathrm{~K}$ & $8.4 \mathrm{~K}$ & $37 \%$ & 32 \\
Old man & $3.40 \mathrm{~K}$ & $2.01 \mathrm{~K}$ & $39 \%$ & 22 \\
Oparara & $14.9 \mathrm{~K}$ & $11.6 \mathrm{~K}$ & $22 \%$ & 140 \\
Theater & $17.35 \mathrm{~K}$ & $14.7 \mathrm{~K}$ & $15 \%$ & 96
\end{tabular}

Table 5.2: Stipple counts from LBG stippling before and after applying our method. 
dark regions, we eliminate any details that might have been present there; we use a conservative default threshold, but for particular images, the user may need to adjust this setting. Detail in light regions is also eliminated through thresholding, but this is of less concern, since so few stipples would have been placed there that such details would not have been communicated in the first place. Our stipple removal through postprocessing is helpful, but imperfect: it might remove stipples that were useful, or fail to remove stipples that could safely have been eliminated. We have sought to avoid wrongly eliminating stipples and largely succeeded by limiting removal to regions with both few stipples and low contrast. In our observation, long bright gradients are the main problematic case; the mac image is an example where too many stipples have been removed, as the shape of the Mac is no longer apparent. We showed this case in Figure 5.11. The result can be improved by assigning a smaller value to the parameter $c$.
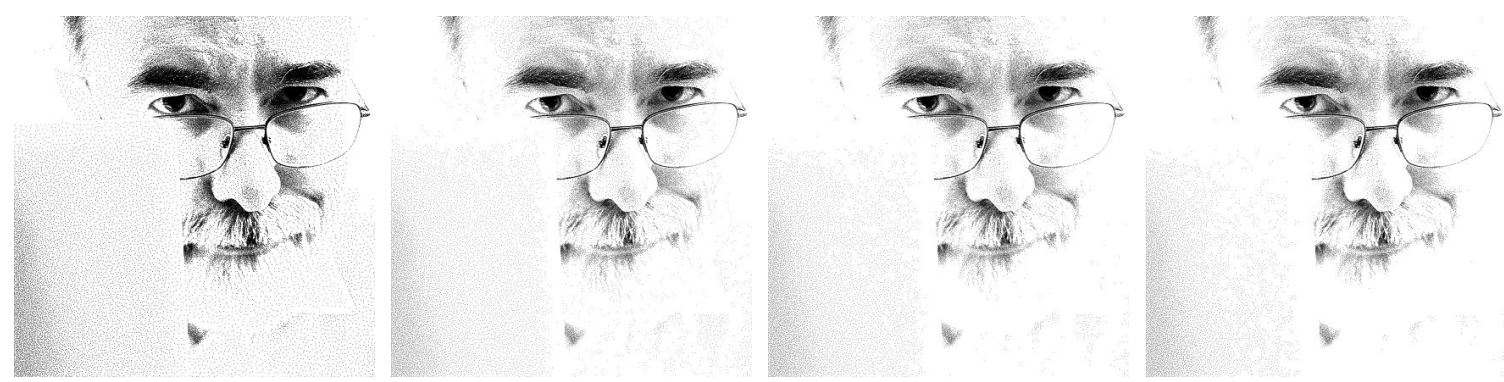

Figure 5.11: Effect of parameter $c$ in bright images. Left to right: original stippling [78], result with $c=3, c=4$, and $c=5$.

Our suggested detail enhancement process is likely the most contentious part of our method. We identified details through computing the residuals from edgepreserving smoothing and then checking whether reversing the residual increased or decreased contrast; this process does not always succeed in improving visibility of details. Incorporating some notion of salience into the computation could be helpful. In addition, reversing the residuals and then stippling does not necessarily reveal the missing detail; the area might still be fairly light, so that too few stipples are placed. Overall, we suggest that more attention should be paid to the problem of stippling light details in light regions. 


\subsection{Summary}

In this chapter, we described a method for improving stippled images in extremetone regions. We threshold images in two directions, setting sufficiently light pixels to maximum intensity and sufficiently dark pixels to minimum intensity; clusters of dark pixels are then represented by polygons rather than by a multitude of stipples. The intensity discontinuities resulting from thresholding are repaired by a Poisson blending operation over a narrow band surrounding each thresholded region. Despite thresholding, the stippling algorithm may still place isolated stipples that contribute little to image content; we postprocess the stipple distribution to remove such stipples.

The resulting images are clearer than the original stippled images, and use fewer stipples, dramatically so when large dark areas are replaced with polygons. Showing detail in light areas remains a problem, though. We suggest a preliminary approach to darken light details in light areas, making them feasible to show with a tonepreserving stippling algorithm; this portion of our method is effective in some cases but we do not recommend applying it blindly to all images. One direction for future work is to further address stipple representation of light details.

We deployed our pre- and postprocessing for structure-preserving stippling as well as LBG stippling. We believe that it would also benefit other stippling algorithms, but have not tested this supposition. Our approach might also be adapted to improve other stroke-based rendering methods or line drawing methods.

This work has been published as follows [7]:

- Rosa Azami, Lars Doyle, and David Mould. "Stipple Removal in Extreme-tone Regions," Expressive (2019), pp. 123-132. 


\section{Chapter 6}

\section{Hybrid Detail Enhancement and Abstraction}

\subsection{Introduction}

Photo abstraction, as practiced in image-based artistic rendering, has traces in the traditional arts. Pablo Picasso, Henri Matisse, Hans Hofmann, and other abstract expressionists tried to connect to viewers through simple but effective visual effects. For example, Matisse's paintings are characterised by flat shapes, controlled lines, and pointillism. In non-photorealistic rendering, abstraction methods often remove highfrequency details from images. Animal fur or whiskers, bumps on rocks, and facial wrinkles are examples of details at risk of being lost through abstraction. Although removing detail is the essence of abstraction, doing so can also be a barrier concealing the real nature of an image. For example, an aged face may not be distinguishable from a younger face. Moreover, textures and details bring believability and vigor to images, as they do to realistic paintings.

We would like to recover and in some cases exaggerate details lost through abstraction. This chapter proposes a hybrid detail enhancement and abstraction process, where an input image is first abstracted with edge-aware filtering, and then a subset of the residual is added back to the filtered image; small residual elements and those further from strong edges are suppressed, while extended elements close to strong edges are enhanced. This process is intended to mimic the artistic notion of indication [49], where image elements such as texture are suggested with scattered details rather than being represented in full.

An unsophisticated filtering process can blur and flatten images. We aim to bring 
excitement and energy to the filtered images by adjusting the brightness locally. We brighten the photograph across the image such that the areas of interest will get increased contrast while colors in the areas of less interest will become lighter and less saturated. We combine the brightening with detail enhancement to improve the emphasis of the augmented details and help the final abstraction to look nicer.

The combination of selective detail enhancement, image brightening, and image abstraction generates a new stylized image.

Our chapter contributions include the following:

- We present an automatic algorithm to locally emphasize image details: details near strong edges are enhanced and those far from edges are reduced.

- We define a system for scoring texture elements, used to establish different levels of texture indication.

- We propose a simple brightening method to be used in conjunction with detail enhancement.

\subsection{Poisson Equation}

The Poisson equation 2.1 has been used broadly in computer vision and image processing. Specifically, it has been used to produce vector fields and for gradient interpolation in image editing systems $[101,107]$. Here, we solve a Poisson equation and use it for both brightening and detail enhancement. We obtain a smooth map over the image considering two main boundary conditions: edges of the image and the ridges of the distance transform map obtained from edges.

The resulting map from solving the Poisson equation provides smooth transitions between the edges and the ridges far from edges. We intend to keep the brightness close to edges intact; such regions mostly introduce main textures of the subjects. However, areas far from edges do not carry significant high-frequency details, so detail can be suppressed here. Figure 6.1 illustrates an example of distance transform map and its smoothed map from solving the Poisson equation.

The smooth map obtained from the Poisson solution can be used to govern detail and brightness adjustment. We aim to generate a stylized photograph by recombining the high-frequency information while avoiding unfavorable overenhancement; Wang 

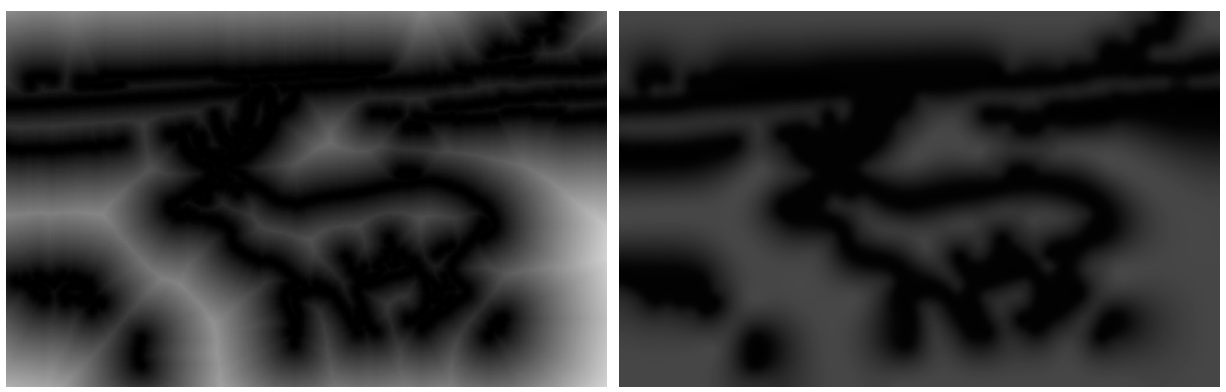

Figure 6.1: An example of distance transform map (left) and its corresponding smooth map obtained from solving a Poisson equation (right).

et al. [132] mention that extreme high-frequency details may result in an unnaturallooking photograph. In next section we present our solution for an automatic but controllable detail and brightness enhancement process.

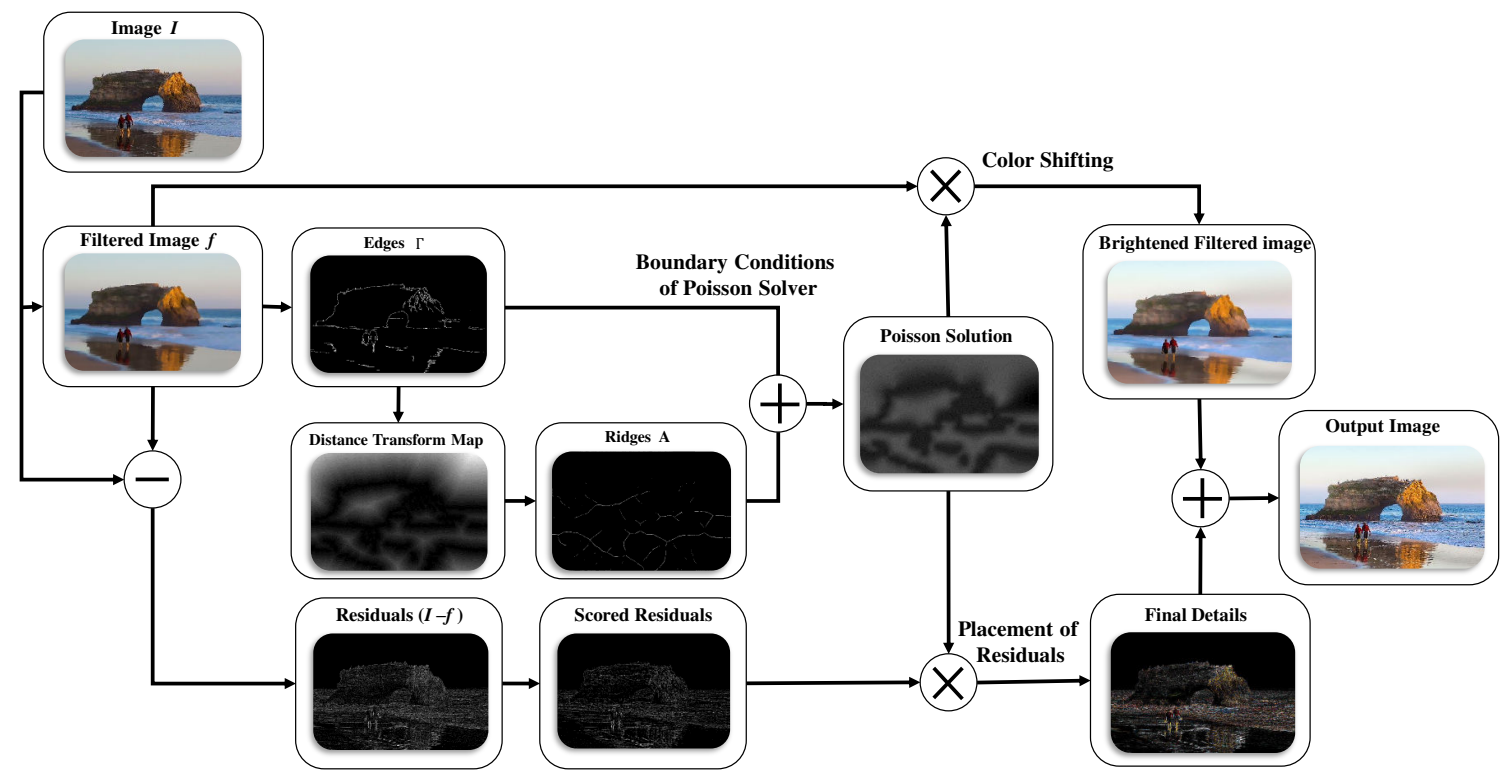

Figure 6.2: Overview of our method. Original rock arch image (C)Vadim Kurland, CC BY 2.0.

\subsection{Methodology}

Our algorithm has several steps. Given an image $I$, we first apply the cumulative range geodesic filter [94], giving us the filtered image. Then, we subtract the filtered 
image from the input image, yielding the residual map $R(x, y)$. To determine which detail elements should be emphasized and which eliminated, the signed residuals undergo additional processing before being recombined with the output image; We use two post-processing steps: sticks filtering [29] and scoring connected components. Next, we solve a Poisson equation to obtain a map that governs the magnitude of brightening and the exaggeration of the residuals. Finally, we combine the selected residuals with the brightened filtered image. Figure 6.2 shows the pipeline of our method with an example image.

\subsubsection{Sticks Filtering}

Extended linear structures, like tree branches or facial wrinkles, are visually important yet may be suppressed even by an edge-preserving filter. In seeking to recover them, the variations in intensity of the residual pixels nearby may make it difficult to distinguish thin features from sparse and disconnected features. To enhance thin and connected line structures, we use the sticks filter, originally designed to reduce speckle noise and preserve linear structures [29].

We use the sticks directions as a decision process. We create a sticks map $T(x, y)$ using Equation 6.1:

$$
\begin{gathered}
T(x, y)=\max _{i \in\{1 . . m\}}\left(\left|\mu\left(s_{i}\right)-\hat{I}_{n}(x, y)\right|\right) \\
\hat{I}_{n}(x, y)=\frac{\sum_{(r, s) \in n \times n} I(x+r, y+s)}{n^{2}} \\
\mu\left(s_{i}\right)=\frac{\sum_{(j, k) \in s_{i}} I(j, k)}{n}
\end{gathered}
$$

where $s_{i}$ is a stick mask, parameter $n$ is the length of each stick, and $m$ is the number of sticks. The sticks direction $T(x, y)$ determines the maximal contrast response, defined as the maximum difference between the average intensity $\mu\left(s_{i}\right)$ of a stick and the average intensity $\hat{I}_{n}(x, y)$ of the neighboring pixels. In our approach we used $n=5$ for $m=8$ sticks orientations. In general, there is a tradeoff between the number of masks and the resolution of orientations; while we would like more resolution, there is a cost in processing time. Our choice of $n=5$ is typical as far as 

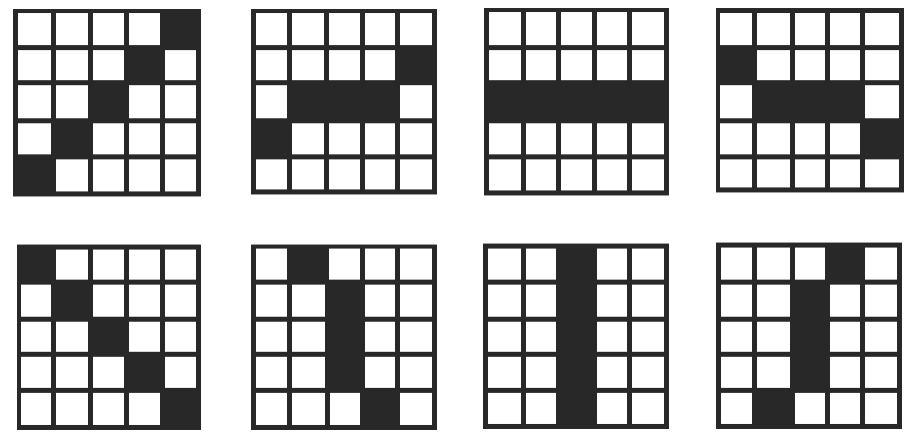

Figure 6.3: The eight masks used for sticks filtering.

we know. Figure 6.3 shows the eight masks from $n=5$.

\subsubsection{Scoring Connected Components}

Textured areas can be characterized by aggregations of high-frequency details, as opposed to sparse or low-frequency details. We estimate whether a given detail is part of a texture by assigning it a score based on the residual content in its neighbourhood.

We convert the grayscale residual map to a binary image and find its connected components [120]. The residual image is thresholded, setting to zero all $R(x, y)$ such that $|R(x, y)|<5$; the nonzero $R(x, y)$ are then considered foreground pixels in the later connected components calculations. Positive and negative components are scored separately. For each connected component $C$, we get a territory (everything within $d$ pixels of any pixel in $C$; we used $d=10$ ) and we sum the magnitudes of all residual pixels with the same sign as pixels in $C$. Thus, large components, with a large territory, as well as smaller components close to areas with large residuals, will receive large scores.

The scores will determine which portions of the residual are emphasized: we will sort the components in order of decreasing score, and the top portion will be exaggerated while the rest are discarded. We normally report the scoring threshold $s_{t}$ as a relative position in the sorted list; e.g., we might discard all but the top $20 \%$, using $s_{t}=0.2$.

Figure 6.4 shows an example of the scored connected components of the positive and negative signed residuals. Using the signed residuals allows us to better control the size of the connected components: individual elements are scored separately, rather than foreground and background being mixed together in a single component 
as might be the case when absolute values are used.
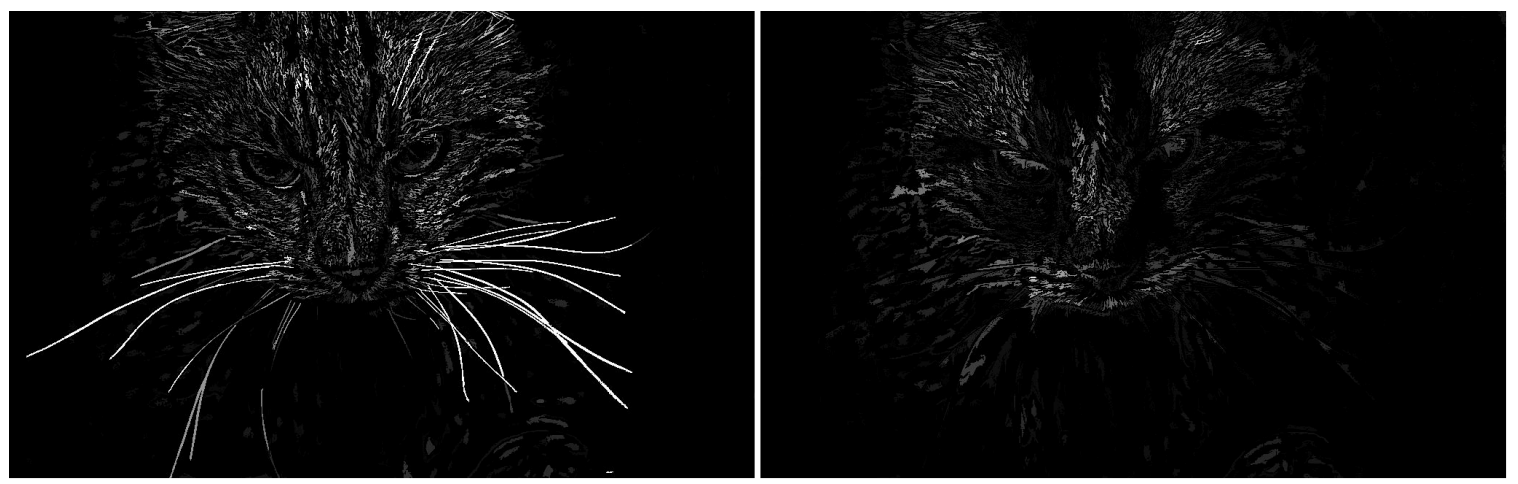

Figure 6.4: Scored connected components. Left: positive residuals; right: negative residuals.

\subsubsection{Poisson Solver}

We plan to preserve the colors close to edges and brighten the image far away from the edges. We will use a Poisson solver to give us a map of where each pixel lies relative to the edges. A possible alternative is to compute the distance from edges directly, but this quantity would not be smooth and could be difficult to normalize properly.

We solve a Poisson equation $F$ over a $2 \mathrm{D}$ domain $\Omega$, with Dirichlet boundary conditions at edges $\Gamma$ set to zero and a fixed value $v$ on the ridges $A$. We extract edges $\Gamma$ using the classical Canny edge detector [20] and the ridges $A$ are effectively the medial axis of the distance transform, that is, the pixels equidistant between edges. Thus, we have the following overdetermined linear system:

$$
\begin{aligned}
& \Delta F(p)=0 \quad p \in \Omega \\
& F(p)=0 \quad p \in \Gamma \\
& F(p)=v \quad p \in A
\end{aligned}
$$

The solution to the above will be a collection of smooth hills, with minimum height at the edges themselves (where the height is zero) and maximum at the ridges between the edges (where the height is $v$, set to 50 in all images unless mentioned otherwise). 


\subsection{Hybrid Abstraction}

We use the map $P$ obtained from solving the above system both to tell us where to brighten the image and where the detail should be maximally enhanced. Brightening is greater where $P(x, y)$ is larger; details are enhanced most where $P(x, y)$ is low and least where $P(x, y)$ is greatest.

\subsubsection{Image Brightening}

The next step is to brighten the filtered image. Brightening the filtered image furthers our goals in two ways. First, the smooth transition of the Poisson map varies the brightness across the image and highlights subjects in focus. Second, a shift or diffusion in colors presents which by generating gradients of colors results in interesting effects. Our color shifting process is defined as follows:

$$
\begin{gathered}
I_{\text {bright }}(x, y)=(1+b \times \Phi(x, y) \times \hat{P}(x, y)) \times I_{\text {filtered }}(x, y) \\
\Phi(x, y)=\min \left(1-I_{r}(x, y), 1-I_{g}(x, y), 1-I_{b}(x, y)\right)
\end{gathered}
$$

where $\hat{P}(x, y)$ is the normalized Poisson solution at each pixel position and $b$ is a user-chosen value that scales the level of brightening. We control the over-brightening of the image based on the values of the color channels for each pixel. In Equation 6.6, $\Phi(x, y)$ is the minimum of $1-c$ over all channels, where $c$ is the normalized color for each channel. In Equation 6.5, we attenuate the colors based on $\Phi(x, y)$. This operation is core of the color shifting process.

In this work, we operated in the RGB colorspace by default, as it is the most commonly used colorspace. We also could use a perceptually uniform colorspace. The result would not be the same; for example, with Lab and Lch colorspaces, we can directly use the luminance channel to alter the brightness. However, if changing the colors is desired, these colorspaces would need new transformation functions. With the RGB model, we have altered the luminosity and colors together. Investigating the effect of other colorspaces would be a good direction for future work. 


\subsubsection{Detail Enhancement}

When we recombine the details with the brightened filtered image, we want to keep the details with higher scores while avoiding to bring back the details with lower scores. We keep the details above a threshold score unchanged, while the details with lower scores will be reduced or eliminated.

Let $T(x, y)$ be the detail map obtained directly from the sticks decision process. We will compute a final detail map $D(x, y)$ governed by a scoring factor $0<s(x, y)<$ 1 , output from the sticks decision process $T(x, y)$, a weight $\lambda(x, y)$ based on the normalized Poisson distances $\hat{P}(x, y)$, and a user-selected constant $k$, controlling the extent of the detail exaggeration. The detail map $D(x, y)$ is computed as follows:

$$
\begin{gathered}
D(x, y)=k \times s(x, y) \times \lambda(x, y) \times T(x, y) \times R(x, y) \\
s(x, y)=\frac{\left(\left|\operatorname{Score}(x, y)-s_{\mathrm{t}}\right|-s_{\mathrm{t}}\right)^{2}}{s_{t}{ }^{2}} \\
\lambda(x, y)=(m-(m-1) \hat{P}(x, y))
\end{gathered}
$$

Parameter $s(x, y)$ in Equation 6.8 is a parabolic downward function that ranges between 0 and 1 ; its domain varies based on the scoring threshold $s_{t}$. If the score of a pixel $(x, y)$ exceeds $s_{t}$ its details will be preserved completely, otherwise the amount of the details to be preserved depends on the value of the $s(x, y)$.

The parameter $\lambda(x, y)$ ranges from a minimum of 1 far from the edges to a maximum of $m$ on the edges. We used $m=3$ for our results. Figure 6.5 shows different detail maps for $m=3, m=4, m=5$, and $m=10$; we can see that the magnitude of $D(x, y)$ increases with larger $m$. Note that both $m$ and $k$ can increase the details, but they work differently: $m$ increases details locally while $k$ acts globally. We set $k=3$ for Figure 6.5.

Finally, we combine the brightened image and the detail map to produce the output:

$$
I_{\text {out }}(x, y)=I_{\text {bright }}(x, y)+D(x, y)
$$

Figure 6.6 shows the effects of filtering and then the outcomes from brightening the filtered image and from computing the detail map. The final image displays the 

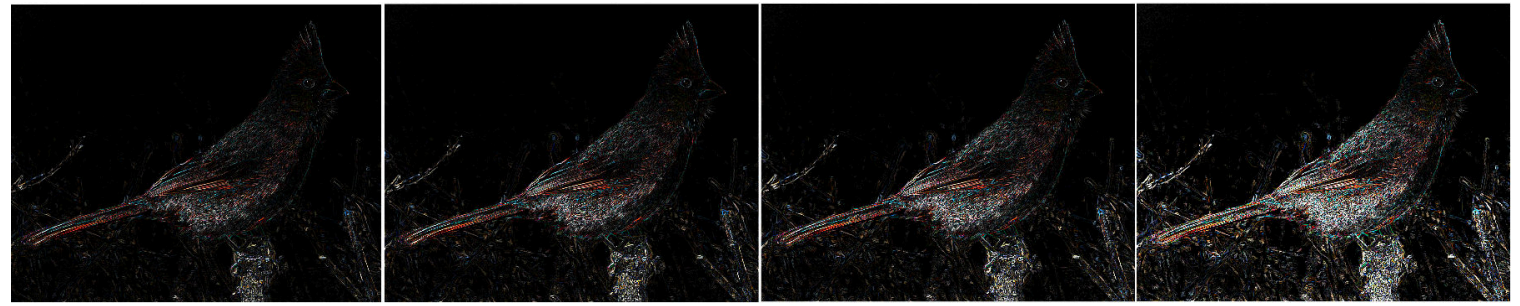

Figure 6.5: Effect of parameter $m$ in $\lambda(x, y)$. Left to right: detail maps for $m=3$, $m=4, m=5$, and $m=10$.

outcome of merging the brightened base image and the detail layer. The filtering process removed the whiskers, eyebrows and many small hairs on the face of the cat. Next, the filtered image was brightened by a factor of $b=2$, with the largest effect on the cat's chest, left shoulder, forehead, and eyes. We set the exaggeration parameter and the score threshold to $k=3$ and $s_{t}=0.5$, respectively. The brightened abstracted image and the detail map are merged in the last step, reintroducing the whiskers and other details on the cat.

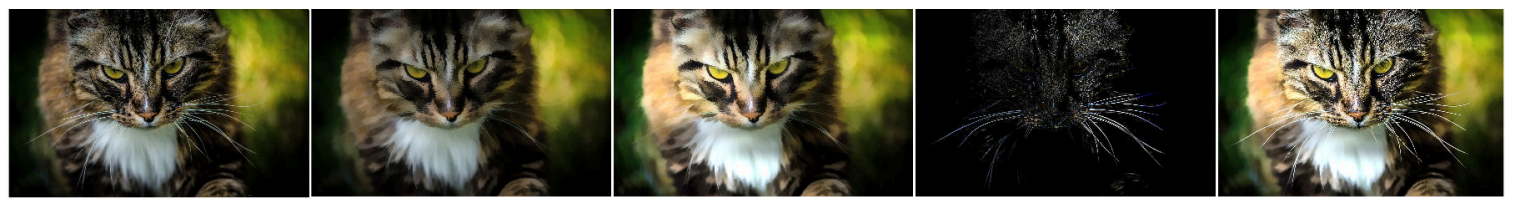

Figure 6.6: Example steps of the enhancement process. Left to right: original image; filtered image; result from brightening the filtered image; detail map; and the final result from merging the brightened image and the detail map. Original Angry cat (C)Peter Pham, CC BY 2.0.
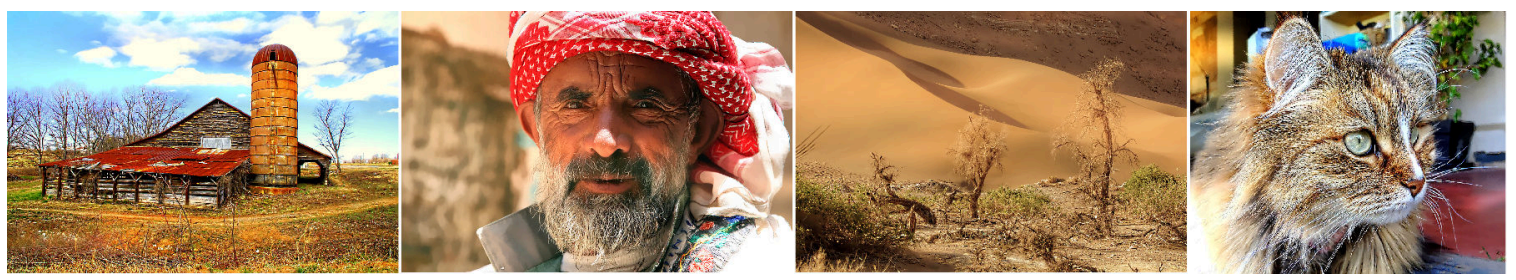

Figure 6.7: Brightened and detail-enhanced images from benchmark set [97] . Left to right: barn, Yemeni, desert and cat.

Our stylization method took advantage of the smooth Poisson solution map to brighten up the images with automatic control over the regions of interest. We created brighter-looking images while focusing the attention on subjects. 
The non-uniform illumination made a shift in colors and created more illustrative and expressive results than the original colors. Changes in brightness are present in all images. An interesting illusion of motion appeared in some results, like the water in swans, whale, and Venice in Figures 6.8 and 6.9. The parameters selected depend on image properties and on the intended result. Moreover, independent parameters allow some manipulation of the results. Figures 6.8 and 6.9 show some representative stylized images from our method. Parameters were set on a per-image basis. The brightness parameter used for the rock arch, old lady, eye, swans and the rock formation was $b=1$; for all others, we set $b=2$. The detail exaggeration parameter set to $k=3$ for all images. The score threshold value was $s_{t}=0.33$ for the Venice, $s_{t}=0.92$ for rock formation, and $s_{t}=0.5$ for the rest.

In whale we can see a small shift in color of the surrounding water, making the whale more distinct from the background. Similarly, the brightness on the face of the old lady shows a cheerful face that emphasizes details, such as the wrinkles. In the eye example, we created a more chromatic iris, yet the skin color around the eye remained normal. Also, the thin vessels on sclera and the eyelashes were preserved well by our detail enhancement process.

In our technique, the more details the original image contains, the more flexibility we have to manipulate them. The Venice, skull rocks, and old lady images contain a significant amount of details. However, we did not add any details to the flattened regions so as to keep the abstract background. The brightening effect and detail enhancement through the Poisson solution are visible in all results. Abstraction has its largest magnitude on the ridges where the Poisson solution has higher values; details are enhanced on edges, where the Poisson solution has its minimum. Note that the abstraction may not be very apparent near ridges, depending on the actual image content; for example, in a pure blue sky, there is no difference between the abstracted (smoothed) image and the original. To alter the balance between abstraction and detail exaggeration, we can vary the threshold of the connected component scores. The most abstracted image was the rock formation, using $s_{t}=0.9$ and hence keeping only a few details. We found the combination of the smooth skull rocks and the sharp features of the shrubs to be quite striking. In waterfall, the tree, surface of the rocks, and green textures are emphasized, making the content of the image more clear.

Figure 6.7 shows results from the benchmark image set [97]. In the barn, the 

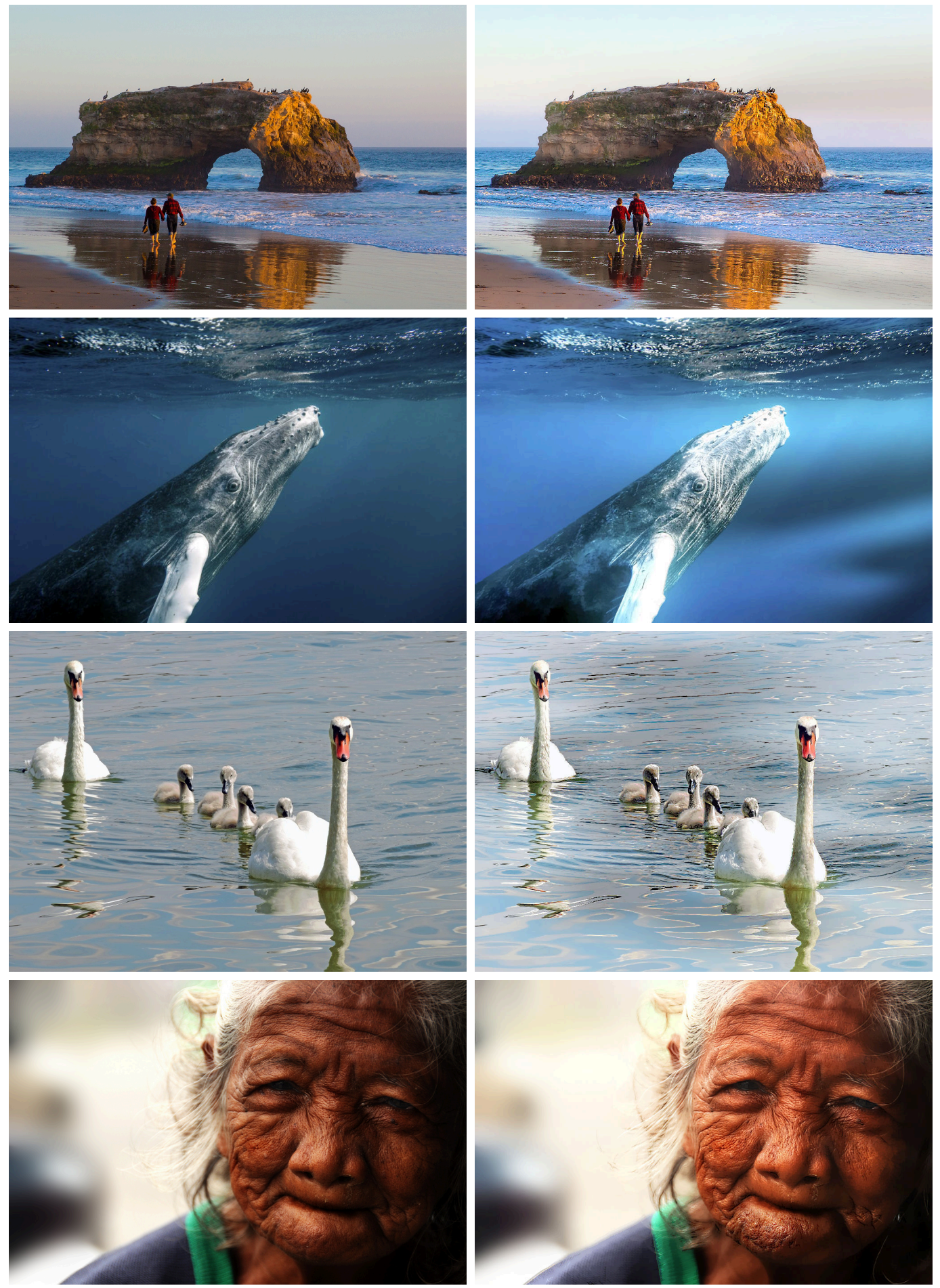

Figure 6.8: Stylization effects. From top to bottom: rock arch, whale, swans and old lady. 

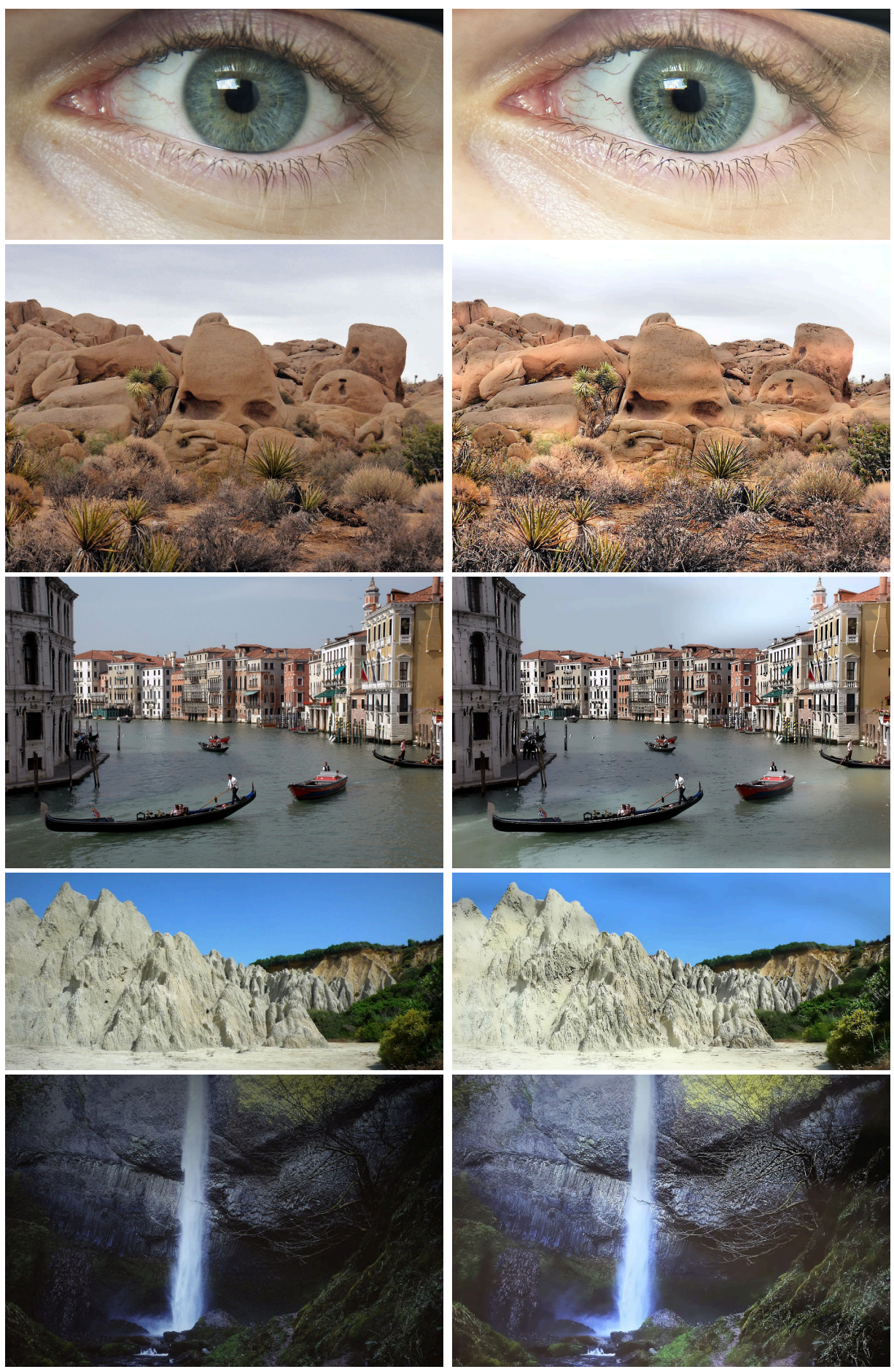

Figure 6.9: Stylization effects (continue). From top to bottom: eye, skull rocks, Venice, rock formation and waterfall. 
details of the barn, trees and some ground textures are enhanced. The beard and wrinkles of the Yemeni face are emphasized. The seared trees of the desert are exposed in front of a brighter background, and the fur and whiskers of the cat are enhanced.

The original Flickr images used in Figures 6.6, 6.12 and 6.15 are illustrated in Figures 6.16; we also used some images from the NPR general benchmark set [97].

\subsubsection{Effect of Parameters}

Our method has three user-chosen parameters with direct influence over the results: the brightening factor $b$, the detail exaggeration factor $k$, and the score threshold $s_{t}$.

We illustrated the effect of the brightening parameter $b$ in Figure 6.10 for $b=1$, and $b=2$. The ceiling of the cave, the surface of the rocks and water are the regions most affected by brightening. However, the darkest area (lower left) does not experience significant changes, owing to the multiplicative brightening process: in general, areas with intensity near zero will not be brightened.
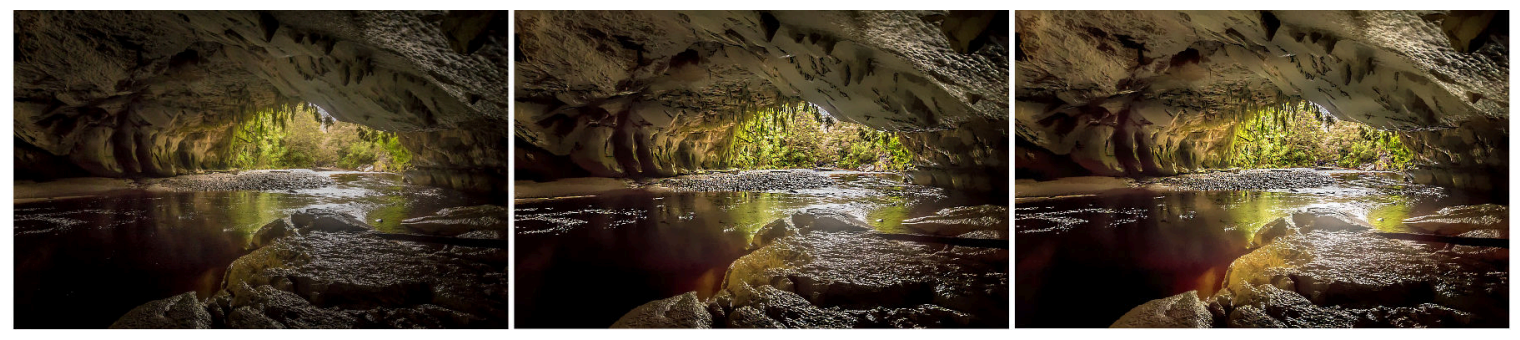

Figure 6.10: Different brightness. Original Oparara image (left) [97], our results with $b=1$ (middle) and $b=2$ (right).

Results from different score thresholds are shown in Figure 6.11. The exaggeration parameter was set to $k=3$ for all results. Choosing a higher threshold removes details with lower scores. From left to right, the first image is the original image, and the remaining images are enhanced with thresholds $s_{t}=0.9, s_{t}=0.75, s_{t}=0.33$ and $s_{t}=0.16$, respectively. In this case, more aesthetic images result from higher thresholds. By reintroducing only a few details, we can reveal the abstraction while emphasizing prominent details of the image.

Figure 6.12 shows results from different detail exaggeration parameters. Small details on the surface of the stones that are not pronounced for $k=3$ become visible 


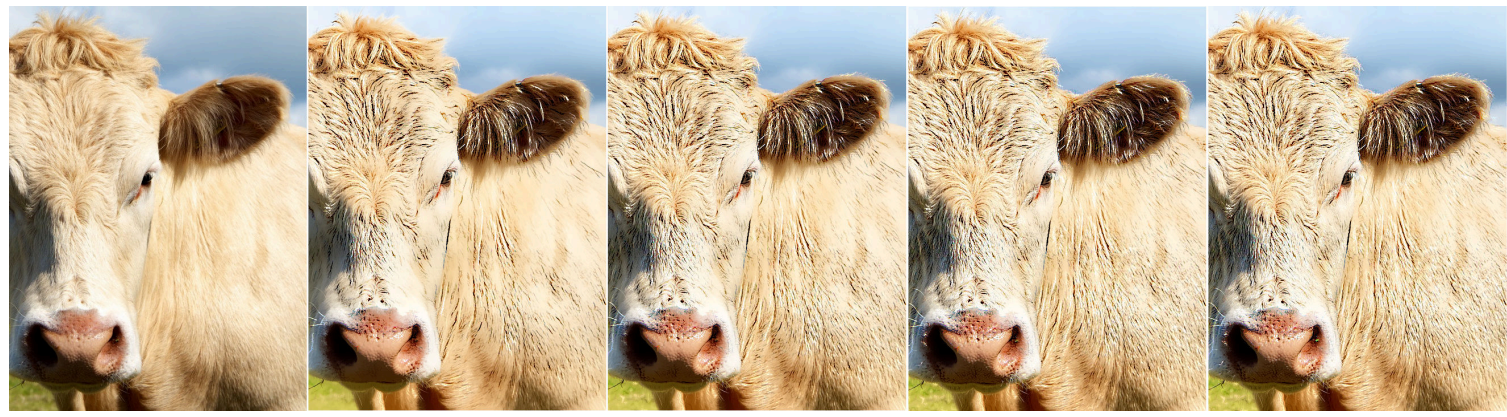

Figure 6.11: Detail enhancement with different thresholding of the sorted connected component scores. Left to right: original, $s_{t}=0.9, s_{t}=0.75, s_{t}=0.33$ and $s_{t}=0.16$. Original cow image (C)Alastair Campbell, CC BY-SA 2.0.

for $k=5$ and $k=7$, emphasizing the roughness of the stone surface. Conversely, while at $k=3$ the stylized cat image is a good mix of abstraction and detail, for higher $k$ the details on the face and forehead become too severe. In extreme cases $(k=7)$, overenhancement of the details manifests visually as noise; noise-like elements appear in the background around the cat's whiskers. In images such as the cat with large-scale coherent texture, more gentle detail exaggeration is warranted.
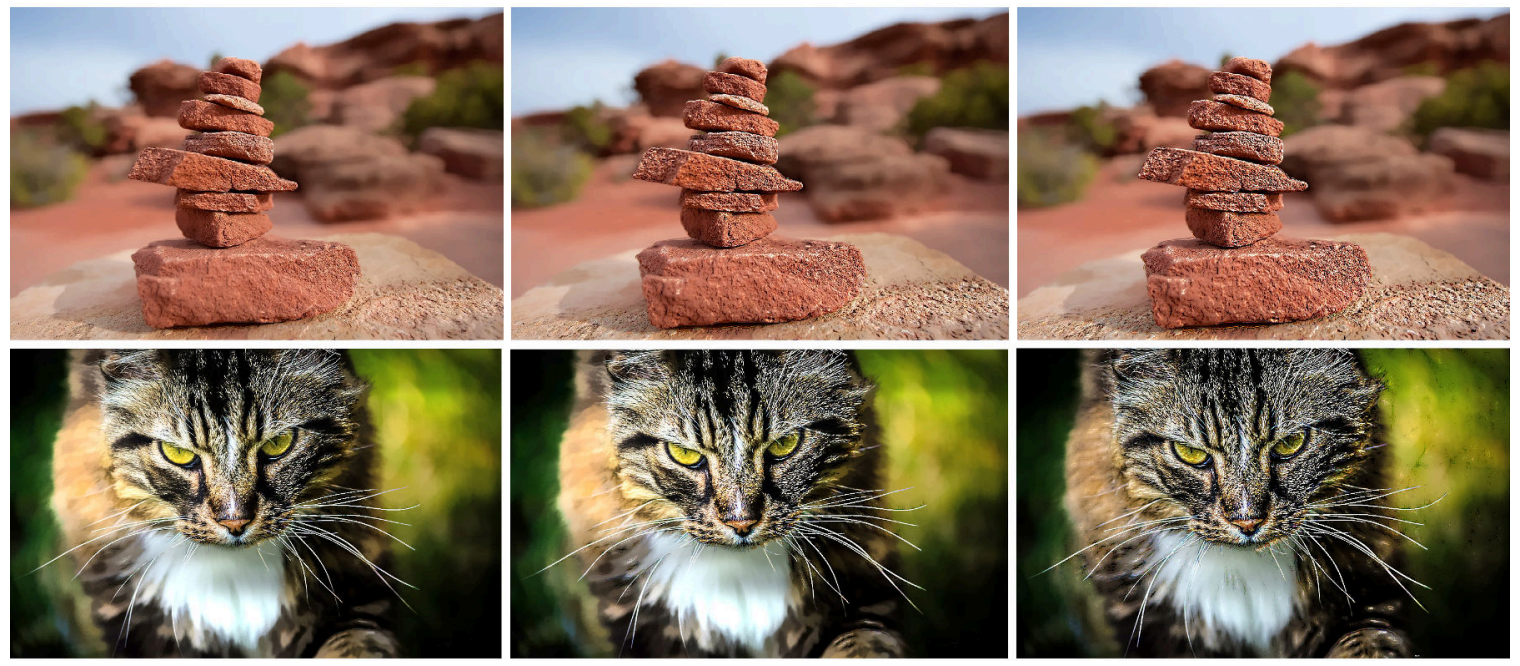

Figure 6.12: Different exaggeration parameters. Left to right: $k=3, k=5$, $k=7$. Original stones image by CanyonlandsNPS (public domain) and angry cat image CPeter Pham, CC BY 2.0. 


\subsubsection{Comparisons}

Our method is akin to the work by Bae et al. [11], who pioneered the base-detail decomposition that we also follow. Our approach can generate a more colorful and brighter image. In order to capture the detail layer they applied the bilateral filter but we chose the cumulative range geodesic filter since we want to partly abstract the image. They magnify high-frequency detail according to an estimate of textureness; our scores can be considered a textureness estimate as well, but we add a second factor to govern the detail amplification, which is the map of distance to a strong edge, enabling us to focus the detail towards the key image elements.

We compare the performance of our algorithm with results from state-of-the-art contrast enhancement methods in Figure 6.13. From left to right we see the original image of the Stanford Memorial Church, the LDR result from Lee et al.'s method [75], gradient domain HDR compression from Fattal et al.'s approach [44], and the result from our algorithm.

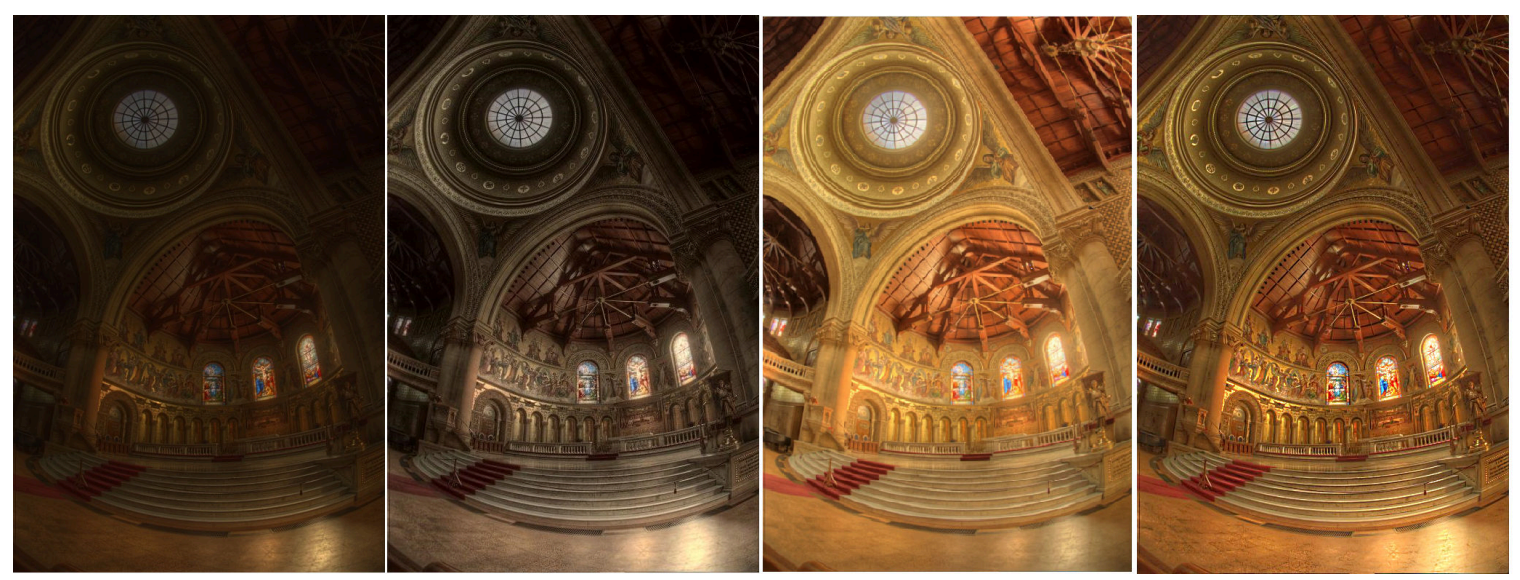

Figure 6.13: Brightness comparison. Left to right: original image of Memorial Church, contrast enhancement of Lee et al. (LDR), gradient domain HDR compression of Fattal et al., our result. Original Stanford Memorial Church image (C)Paul Debevec.

The result by Fattal et al. is brighter and more colorful than that from Lee et al. The details are not emphasized everywhere, as we can see, for example, on the small and large arcs. The result from Lee et al. preserves the details well in the brighter area, while in dark regions on the ceiling, it loses visibility. The colors are dark and stuffy and it does not convey the original colors. 
In our result, the contrast is enhanced naturally and the details are well preserved almost everywhere. The reader is encouraged to zoom in to better see the differences. The colors are much brighter than either the originals or the result from Lee et al. and the dark hidden regions showed up with a good amount of details. While Fattal et al.'s result is brighter than ours, the colors are faded, and the artworks on the ceilings and walls are barely visible. In general, our result has a higher contrast than Fattal et al.'s. In addition, the glasses, fence, and stairs are better emphasized than in Fattal et al.'s result. Note that in order to achieve a uniform level of detail enhancement, we could omit the local variation of detail enhancement.

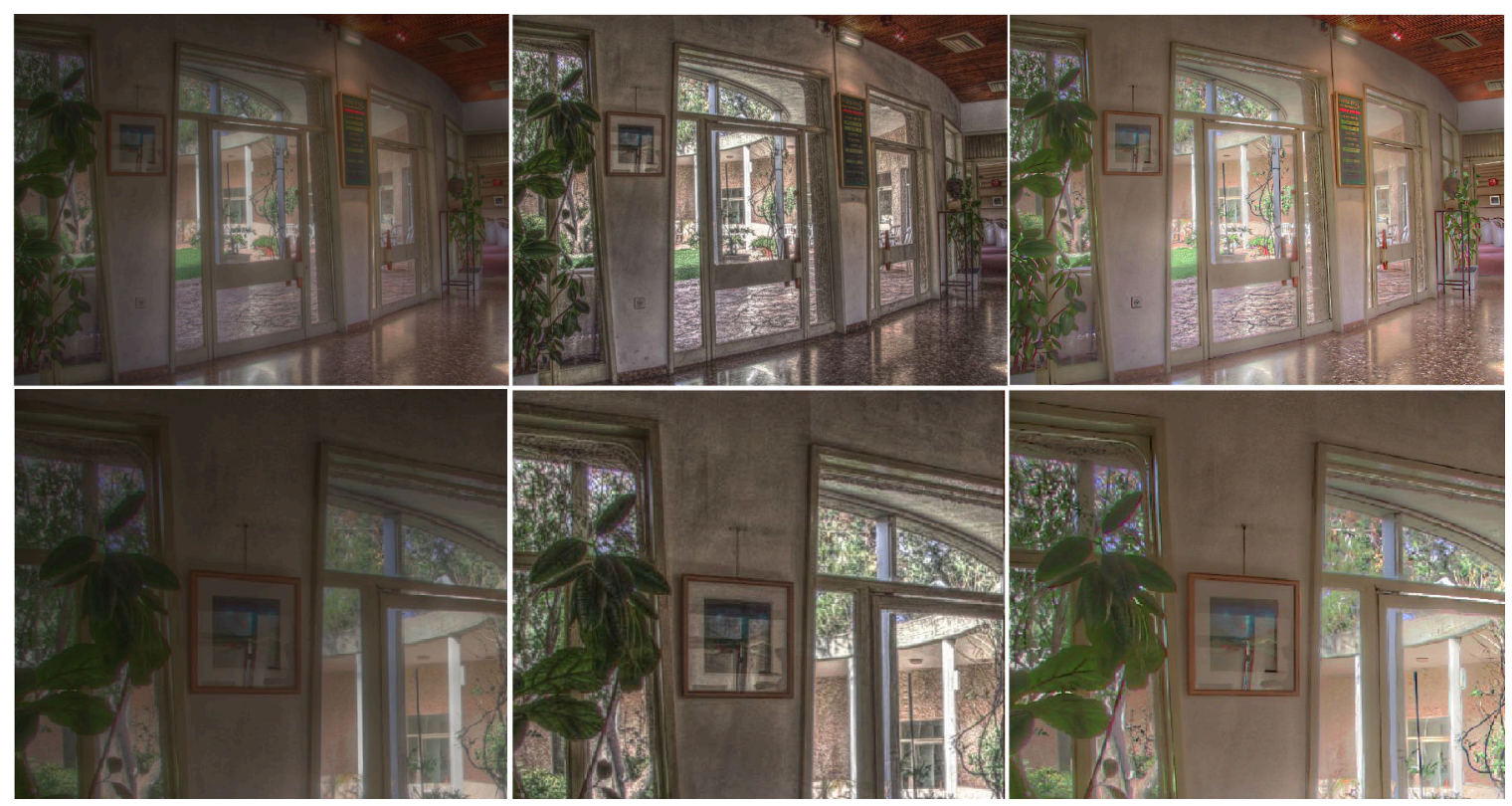

Figure 6.14: Comparison of detail enhancement. Top row, left image: original image; middle: local Laplacian filter of Paris et al.; right: our result. The bottom row shows a close-up of the wall. Original Belgium House image CRaanan Fattal.

Finally, we compare our result with the local Laplacian filters of Paris et al. [105]; see Figure 6.14. Although both local Laplacian filtering and the proposed algorithm brightened the original image, our algorithm provides better visibility in some regions. For instance, in the right corner of the room, there is a plant and a statue on a long table in front of it, which is unclear in the local Laplacian result. Also, the overenhanced result from local Laplacian filtering exaggerates the noise on the wall as is apparent in the close-up images in the bottom row. 


\subsubsection{Timing}

The computation time varies linearly with the number of pixels. Our algorithm takes about 3 minutes to process a $1024 \times 640$ image on a laptop Intel(R) Core(TM) i7$4510 \mathrm{U}$ with a $2.6 \mathrm{GHz} \mathrm{CPU}$ and 16.0 GB of RAM. Filtering and solving the linear system of the Poisson equation takes the majority of the time. The cumulative range geodesic filter takes less than a minute to process an image with a mask size of 500 and 22 seconds to process with a mask size 200. Solving the linear system is the most time consuming part of our algorithm, requiring almost 2 minutes. Our implementation is not optimized and there is a great deal of room to improve the speed. We used a naive edge detection in our work, while a cleaned up edge detection can decrease the number of edge pixels and solve the linear system faster.

\subsubsection{Limitations}

We experienced some failure cases. The most common failure case for our method is the poor color shifting in regions far away from edges, such as the sky. The sky in Venice in Figure 6.15 is an example of this case. This deficiency could be improved by choosing a larger value for $v$ in Poisson solver. We demonstrated an improved outcome for the Venice sky in Figure 6.9 using $v=50$, while using $v=20$ in Figure 6.15. Another possible direction to remedy this problem would be using a flexible value for $v$, such that the value of $v$ on the ridge changes with respect to its distance transform value.

Another cause of failure is preserving too much texture; this depends on the choice of the input image. Images with many close edge points preserve most of the texture from the original image. We want the output result to partially contain the background abstracted image, so that the detail map shows the effect of enhancement. The rocks, ocean and grass of Etretat in Figure 6.15 show this issue; while overall it looks nice, the abstraction effect can hardly be seen. We may be able to address this issue with more sophisticated edge detection that can eliminate the redundant edges.

We set a global parameter for the edge detection, which may not be effective for all images. An image where different regions contain different levels of contrast will pose problems for the global parameters of the Canny edge detector. Some details will be missed and portions of the image will remain blurry. Further, the brightening process will be affected, washing out colors in such regions. In general, an adaptive 
edge detector can be more effective. However. the Canny edge detector does a good job for many images; we did not explore the use of adaptive edge detection, which is a possible direction of future work.

Also, a coarse transition of colors between adjacent pixels is perceived as noise in some images. This could be due to clamping of the colors in different channels. To address this issue we need a smooth transition in shifting the colors after augmenting the detail map; also, using a different color space may help with this limitation. In Figure 6.15 the noisy line of the fingers and the line above the shoulder of the old man shows this problem.
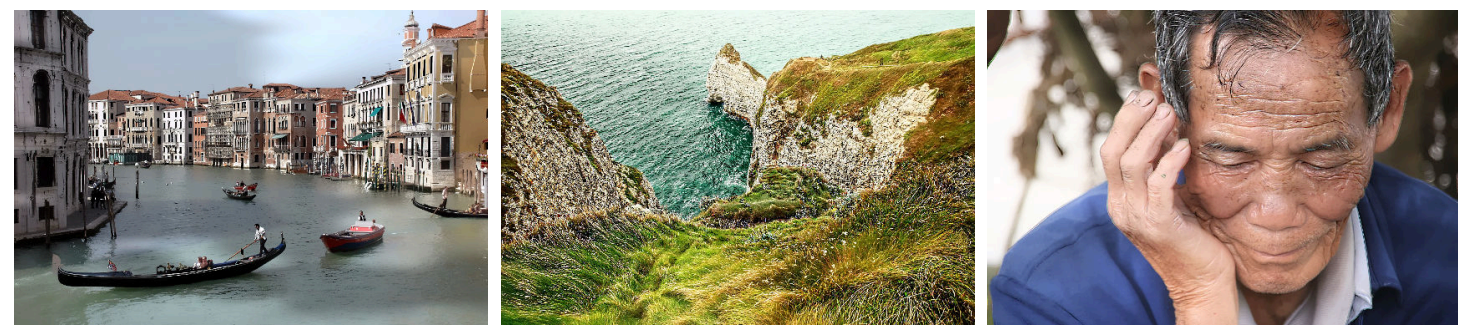

Figure 6.15: Failure cases. Strong color shifts of sky in Venice; presence of many textures in Etretat hiding the abstract background; noise effect in Chinese man next to the edges.
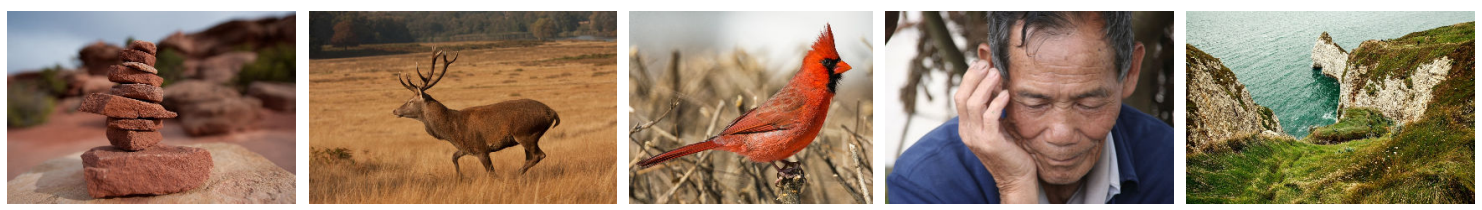

Figure 6.16: Original Flickr images used in this chapter. From upper left: stones by CanyonlandsNPS (public domain), northern cardinal (C)Bill Damon, CC BY 2.0, Chinese (C)Anja Disseldorp, CC BY 2.0, Etretat (C)Cristian Bortes, CC BY 2.0 and

\subsection{Summary}

In this chapter, we addressed the problems of abstraction methods in eliminating the detail from the image and producing dull colors due to averaging; two common problems that have appeared in both region-based and filter-based abstractions. In 
chapters 3 and 4 we have provided solutions to address these problems in region-based abstraction. This chapter presented an automated image manipulation technique that provides a blend of detail and abstraction on a filtered image. In regions of the image near strong edges, assumed to represent relevant detail, we exaggerate detail, and in regions more distant from strong edges, we allow the abstraction effect to dominate.

Additionally, we used a mechanism to brighten the image far from the edges, preserving the original image colors near the edges. The net effect is a cheerful image in which some details are emphasized while others are suppressed. In comparison with the state-of-the-art contrast enhancement methods, our result shown the contrast is enhanced naturally and the details are well preserved almost everywhere.

This work has been published as follows [8]:

- Rosa Azami and David Mould. "Detail and Color Enhancement in Photo Stylization," In Proc. CAe (2017), pp. 1-11. 


\section{Chapter 7}

\section{Conclusions and Future Work}

This thesis seeks answers to some problems that appear in NPR abstraction methods. The challenge resides in presenting the essential component of the image while the abstraction occurs. It is the essence of most abstraction methods to compromise the image details and colors; however, the presence of details and specific color assignments can create artistic renderings.

In this thesis, we have provided detailed stylized abstractions from photographs, where we often used the region as the main element of the abstractions.

Regions can simplify the image into semi-semantic structures, which can be employed for further abstraction or stylization. Often we have seen uniformity in shape and size of the textures in abstractions, which could be the product of a filtering mechanism or an underlying segmentation in a region-based abstraction. In this regard, we provided an abstraction algorithm that captures regions with high irregularity in shape and size using a growth mechanism (Chapter 3). By treating regions as a form of complex strokes, we retained many details of the image that are normally removed through the abstraction process.

Specific colorization can turn a dull abstraction into a vivid image. The flat colorization of abstractions is a common issue of the averaging process. Using regions as the primitive of the abstraction, we introduced a recoloring method (Chapter 4) to generate expressive abstractions.

Additionally, we manipulated regions of interest in tone-preserving stipplings [31, $78]$ to improve stippling representation in the extreme-tone regions. These methods require enormous numbers of stipples to represent the black areas of an image, and often in the light regions, they present unnecessary stipples which do not contribute to image structure. Our proposed algorithm in (Chapter 5) improved the representation 
of the stippling in such regions.

Furthermore, we explored the idea of presenting the image detail in filter-based abstraction. Abstraction by filtering process often simplifies images by removing high-frequency details like textures or thin features during the process, while they can bring believability and vigor to images. Our hybrid detail enhancement and abstraction process (Chapter 6) locally emphasizes details lost through abstraction.

\subsection{Conclusions}

The methods presented in this thesis have considered edge preservation, contrast awareness, colorfulness and detail enhancement to create abstractions. The following conclusions are drawn from these concepts and techniques presented in this thesis.

Highly irregular oversegmentation. We provide a highly detailed abstraction of images through regions of irregular shape and sizes, where the image plane is covered by overlapping irregularly shaped regions that approximate the image content. The final result has reduced detail, befitting abstraction, but still contains some small structures such as highlights; thin features and crooked boundaries are retained, while interior details are softened, yielding a painting-like abstraction effect. Further abstraction is produced through simplifying the region boundaries and a simple recoloring of the regions. In particular, compared with other over-segmentation methods [2], the proposed method has advantages in capturing the regions with complex shapes and sizes.

Vivid recoloring of abstraction. Using region-based abstraction as a framework for stylization, we presented a recoloring method for expressive colorization of abstractions. The algorithm takes an over-segmented image and a palette, then it assigns a color to each region. The algorithm designed based on the widest path problem allowed to maintain the image contrast and objects' recognizability. Multiple variants of stylizations demonstrated the flexibility of the design for employing further changes to the algorithm. The algorithm is not limited to the over-segmentation provided by this thesis and can be employed by other methods.

Improving the stipple presentation in extreme-tone regions. We considered regions as one of the main primitives in image representation, which opened up possibilities for improving the stippled images in extreme-tone regions. The suggested 
method identifies the dark regions of the image and covers them with solid polygons instead of stipples. It eliminates the discontinuities around polygons and the nearby regions by employing a Poisson blending operation over a narrow band surrounding each polygon. To control stipple placement in light regions, the method eliminates unnecessary stipples in light regions, both through preprocessing (thresholding) to prevent stipple placement in very light areas and post-processing to identify and remove such stipples. The resulting images became clearer than the original stippled images and had dramatically fewer stipples. Deploying the method on LBG stippling [31] showed that other stippling algorithms might benefit from this approach. Further, a preprocessing step is applied to the image to better show small details, measure local contrast, and increase it for selected elements.

Detail enhancement and color manipulation in abstraction. Abstractions produced by filtering methods lack details and colorfulness as a natural result of the averaging process. An automatic system is presented for photo manipulation, which brightens a filtered image and alters the detail levels. The method uses a residual layer to reintroduce and exaggerate details in areas near strong edges and a scoring mechanism determines which detail elements should be emphasized and which eliminated. Here, a smooth map is calculated from a Poisson equation to govern the magnitude of brightening and exaggerating the residuals. This method locally emphasizes image details and mimics the artistic notion of indication. Comparisons with state-of-the-art contrast enhancement methods revealed that the images' contrast is naturally enhanced and details are well preserved almost everywhere.

\subsection{Future Work}

There are multiple potential research aspects to consider for extending the work in the future:

- Stylized animations An extension to the region-based abstraction method would be applying the method to video, computing regions in a 3D graph rather than 2D. Then render the frames by slicing the 3D blobs. However, we have an initial implementation of this step, the extracted regions from consequent frames lack coherency due to blurriness caused by motion. Considering a method to remove the outlier surface boundaries, we may get more fitted 3D volumes to the regions. In the same direction, other possibilities would be producing 3D photos 
(i.e. Facebook 3D photos) or 3D paintings. 3D paintings have become popular in VR applications. This concept could be extended to an interactive 2D screen, where regions could be rendered with different stroke shapes in different depths registered from multiple frames.

- Palette manufacturing In our region recoloring approach, we have used fixed given colors for colorization, while an algorithm could be designed to produce palettes with regard to the image colors, or given an initial set of colors, it could augment the colors of the palette. Such an approach may need a particular study on the psychology of color combination and the color harmony theory. The mixture of palettes is another possible direction in this topic. The mixture of colors for recoloring the region-based abstraction would need to include the region constraints, which makes it a difficult problem and good follow-up research.

- The widest path algorithm in NPR The widest path algorithm and its possible usage are not well studied in the computer vision and image processing field. Employing the widest path algorithm, hopefully, can drive some ideas into the field of NPR. For example, we are interested in discovering the effect of this algorithm on geodesic filtering or min-cut algorithm; where it could be a substitute for the shortest path algorithm or max-flow of the graph, respectively.

- Textures interpolations with regions interior A possible future work could be designing a stylization method that infuses texture into the region-based abstraction. This concept has been applied locally to masked regions in an image plane [107] or uniformly segmented image [37], yet there is a potential for investigating this idea for highly irregular regions.

Lastly, there is room for improvement in both region-based abstraction and colorization of the over-segmented regions. In region-based abstraction, it should be possible to obtain equally complex results with fewer seeds, saving some computation time. We would like to experiment with different distance metrics for the initial region calculation, using not only color in the incremental distance but also other image properties, including larger-scale features such as histograms of gradients.

In our recoloring mechanism, the constraints on the sign of the luminance change helped with contrast preservation. However, a problem occurs when two similar 
regions are not neighbors, they may receive different colors (e.g., a sky area may be broken up by branches). A possible cause of this issue could be the widest path decision on compromising the weak edges. We can investigate the widest path mechanism on such edges in the future. Finally, we would like to design a more comprehensive weight function for the graph's edges which considers factors such as the spatial distance between regions and the count of shared contours.

It is hoped these methods will accommodate the growth of new algorithms in Non-Photorealistic Rendering. 


\section{List of References}

[1] "Abstract realism - the artists guild painting \& drawing classes," Feb 2020. [Online]. Available: https://brightlightfineart.com/abstract-realism/

[2] R. Achanta, A. Shaji, K. Smith, A. Lucchi, P. Fua, and S. Süsstrunk, "SLIC superpixels compared to state-of-the-art superpixel methods," IEEE Trans. Pattern Anal. Mach. Intell., vol. 34, no. 11, pp. 2274-2282, Nov 2012.

[3] —, "SLIC superpixels," Technical report, EPFL, p. 15, 062010.

[4] E. Aharoni-Mack, Y. Shambik, and D. Lischinski, "Pigment-based recoloring of watercolor paintings," in NPAR '17, Proceedings of the Symposium on NonPhotorealistic Animation and Rendering, H. Winnemöller and L. Bartram, Eds. New York, USA: ACM, 2017.

[5] N. Ahuja, "A transform for multiscale image segmentation by integrated edge and region detection," IEEE Trans. Pattern Anal. Mach. Intell., vol. 18, no. 12, pp. 1211-1235, 1996.

[6] P. Arbelaez, M. Maire, C. Fowlkes, and J. Malik, "From contours to regions: An empirical evaluation," in 2009 IEEE Conference on Computer Vision and Pattern Recognition, 2009, pp. 2294-2301.

[7] R. Azami, L. Doyle, and D. Mould, "Stipple removal in extreme-tone regions," in $A C M / E G$ Expressive Symposium, C. S. Kaplan, A. Forbes, and S. DiVerdi, Eds. The Eurographics Association, 2019.

[8] R. Azami and D. Mould, "Detail and color enhancement in photo stylization," in Proceedings of the Symposium on Computational Aesthetics, ser. CAE '17. ACM, 2017, pp. 1-11.

[9] — "Image abstraction through overlapping region growth," in Proceedings of Graphics Interface 2020, ser. GI 2020. Canadian Human-Computer Communications Society / Société canadienne du dialogue humain-machine, 2020, pp. $66-73$.

[10] - "Artistic recoloring of image oversegmentations," in Graphics Interface 2021, 2021. [Online]. Available: https://openreview.net/forum?id= z66fCE6_Ja0

[11] S. Bae, S. Paris, and F. Durand, "Two-scale tone management for photographic look," in ACM SIGGRAPH 2006 Papers, ser. SIGGRAPH '06. ACM, 2006, pp. 637-645. 
[12] P. Barla, S. Breslav, L. Markosian, and J. Thollot, "Interactive Hatching and Stippling by Example," INRIA, Research Report RR-6461, 2006. [Online]. Available: https://hal.inria.fr/inria-00084569

[13] S. Beucher and F. Meyer, "The morphological approach to segmentation: The watershed transformation," in Mathematical Morphology in Image Processing, 01 1993, vol. Vol. 34, p. 433-481.

[14] M. Bohra and V. Gandhi, "ColorArt: Suggesting colorizations for graphic arts using optimal color-graph matching," in GI 2020, Proceedings of Graphics Interface. Canadian Human-Computer Communications Society / Société canadienne du dialogue humain-machine, 2020, pp. 95 - 102.

[15] I. Boyadzhiev, K. Bala, S. Paris, and E. Adelson, "Band-sifting decomposition for image-based material editing," ACM Trans. Graph., vol. 34, no. 5, pp. 11-16, Nov 2015.

[16] Y. Boykov and G. Funka-Lea, "Graph cuts and efficient n-d image segmentation," International Journal of Computer Vision, vol. 70, no. 2, pp. 109-131, Nov 2006.

[17] Y. Boykov and M.-P. Jolly, "Interactive graph cuts for optimal boundary region segmentation of objects in N-D images," in Proceedings Eighth IEEE International Conference on Computer Vision. ICCV 2001, vol. 1, 2001, pp. 105-112 vol.1.

[18] W. Burger and M. J. Burge, Principles of Digital Image Processing. SpringerVerlag London 2013, 1993, vol. 5, ch. 6, pp. 169-227.

[19] B. Cabral and L. C. Leedom, "Imaging vector fields using line integral convolution," in Proceedings of the 20th Annual Conference on Computer Graphics and Interactive Techniques, ser. SIGGRAPH '93. New York, USA: ACM, 1993, pp. 263-270.

[20] J. Canny, "A computational approach to edge detection," IEEE Transactions on Pattern Analysis and Machine Intelligence, vol. PAMI-8, no. 6, pp. 679-698, 1986.

[21] W. Casaca, M. Colnago, and L. G. Nonato, "Interactive image colorization using Laplacian coordinates," in Computer Analysis of Images and Patterns, G. Azzopardi and N. Petkov, Eds. Cham: Springer International Publishing, 2015, pp. 675-686.

[22] H. Chang, O. Fried, Y. Liu, S. DiVerdi, and A. Finkelstein, "Palette-based photo recoloring," ACM Trans. Graph., vol. 34, no. 4, pp. 1-11, Jul 2015.

[23] J. Chang, B. Alain, and V. Ostromoukhov, "Structure-aware error diffusion," ACM Trans. Graph., vol. 28, no. 5, pp. 162:1-162:8, 2009.

[24] D. Chen, L. Yuan, J. Liao, N. Yu, and G. Hua, "Stylebank: An explicit representation for neural image style transfer," in 2017 IEEE Conference on Computer Vision and Pattern Recognition (CVPR), 2017, pp. 2770-2779. 
[25] D. Comaniciu and P. Meer, "Mean shift: A robust approach toward feature space analysis," IEEE Trans. Pattern Anal. Mach. Intell., vol. 24, no. 5, pp. 603-619, May 2002.

[26] M. Couprie and G. Bertrand, "Topological gray-scale watershed transformation," in Vision Geometry VI, R. A. Melter, A. Y. Wu, and L. J. Latecki, Eds., vol. 3168, International Society for Optics and Photonics. SPIE, 1997, pp. 136 $-146$.

[27] J. Cousty, L. Najman, Y. Kenmochi, and S. Guimarães, "Hierarchical segmentations with graphs: Quasi-flat zones, minimum spanning trees, and saliency maps," Journal of Mathematical Imaging and Vision, vol. 60, no. 4, pp. 479$502,2018$.

[28] A. Criminisi, T. Sharp, C. Rother, and P. P'erez, "Geodesic image and video editing," ACM Trans. Graph., vol. 29, no. 5, pp. 1-15, Nov 2010.

[29] R. Czerwinski, D. Jones, and W. O'Brien, "Detection of lines and boundaries in speckle images-application to medical ultrasound," IEEE Transactions on Medical Imaging, vol. 18, no. 2, pp. 126-136, 1999.

[30] D. DeCarlo and A. Santella, "Stylization and abstraction of photographs," in Proceedings of the 29th Annual Conference on Computer Graphics and Interactive Techniques, ser. SIGGRAPH '02. New York, USA: ACM, 2002, pp. 769-776.

[31] O. Deussen, M. Spicker, and Q. Zheng, "Weighted Linde-Buzo-Gray stippling," ACM Trans. Graph., vol. 36, no. 6, pp. 233:1-233:12, Nov 2017.

[32] O. Deussen, S. Hiller, C. Van Overveld, and T. Strothotte, "Floating points: A method for computing stipple drawings," Computer Graphics Forum, 2000.

[33] O. Deussen and T. Isenberg, "Halftoning and stippling," Image and Video based Artistic Stylisation, Springer, vol. 42, pp. 45-61, 2013.

[34] O. Deussen and T. Strothotte, "Computer-generated pen-and-ink illustration of trees," in Proceedings of the 27th Annual Conference on Computer Graphics and Interactive Techniques, ser. SIGGRAPH '00. ACM Press/Addison-Wesley Publishing Co., 2000, pp. 13-18.

[35] S. DiVerdi, J. Lu, J. Echevarria, and M. Shugrina, "Generating Playful Palettes from images," in Expressive '19, ACM/EG Expressive Symposium, C. S. Kaplan, A. Forbes, and S. DiVerdi, Eds. Goslar, DEU: Eurographics Association, 2019.

[36] D. H. Douglas and T. K. Peucker, Algorithms for the Reduction of the Number of Points Required to Represent a Digitized Line or its Caricature. John Wiley \& Sons, Ltd., 1973, ch. 2, pp. 15-28.

[37] L. Doyle, F. Anderson, E. Choy, and D. Mould, "Automated pebble mosaic stylization of images," Computational Visual Media, vol. 5, no. 1, pp. 33-44, Mar. 2019. 
[38] L. Doyle and D. Mould, "Painted stained glass," in Proceedings of the Joint Symposium on Computational Aesthetics and Sketch Based Interfaces and Modeling and Non-Photorealistic Animation and Rendering, ser. Expresive '16. Eurographics Association, 2016, pp. 1-10.

[39] F. Durand and J. Dorsey, "Fast bilateral filtering for the display of highdynamic-range images," ACM Trans. Graph., vol. 21, no. 3, pp. 257-266, Jul. 2002.

[40] M. Elad, "On the origin of the bilateral filter and ways to improve it," Trans. Img. Proc., vol. 11, no. 10, pp. 1141-1151, Oct. 2002.

[41] J. H. Elder and R. M. Goldberg, "Image editing in the contour domain," IEEE Transactions on Pattern Analysis and Machine Intelligence, vol. 23, no. 3, pp. 291-296, 2001.

[42] L. Fang, J. Wang, G. Lu, D. Zhang, and J. Fu, "Hand-drawn grayscale image colorful colorization based on natural image," The Visual Computer, vol. 35, no. 11, pp. 1667-1681, Nov 2019. [Online]. Available: https://doi.org/10.1007/s00371-018-1613-8

[43] N. Faraj, G.-S. Xia, J. Delon, and Y. Gousseau, "A generic framework for the structured abstraction of images," in Proceedings of the Symposium on NonPhotorealistic Animation and Rendering, ser. NPAR '17. New York, USA: ACM, 2017, pp. 1-11.

[44] R. Fattal, D. Lischinski, and M. Werman, "Gradient domain high dynamic range compression," ACM Trans. Graph., vol. 21, no. 3, pp. 249-256, Jul 2002.

[45] P. F. Felzenszwalb and D. P. Huttenlocher, "Efficient graph-based image segmentation," International Journal of Computer Vision, vol. 59, no. 2, pp. 167$181,2004$.

[46] L. A. Gatys, A. S. Ecker, and M. Bethge, "A neural algorithm of artistic style," CoRR, vol. abs/1508.06576, 2015.

[47] T. Gerstner, D. DeCarlo, M. Alexa, A. Finkelstein, Y. Gingold, and A. Nealen, "Pixelated image abstraction," in NPAR 2012, Proceedings of the 10th International Symposium on Non-photorealistic Animation and Rendering, Jun. 2012.

[48] G. R. Greenfield and D. H. House, "Image recoloring induced by palette color associations," Journal of WSCG, vol. 11, pp. 189-196, 2003.

[49] A. L. Guptill, Rendering in Pen and Ink: The Classic Book On Pen and Ink Techniques for Artists, Illustrators, Architects, and Designers. Potter/TenSpeed/Harmony, 2014.

[50] P. Haeberli, "Paint by numbers: abstract image representations," SIGGRAPH Comput. Graph, vol. 24, no. 4, pp. 207-214, Sep 1990.

[51] P. Hall and A. Lehmann, "Don't measure - appreciate! NPR seen through the prism of art history," in Image and Video-Based Artistic Stylisation, P. L. Rosin and J. P. Collomosse, Eds. Springer, 2013, pp. 333-351. 
[52] A. Hausner, "Simulating decorative mosaics," in Proceedings of the 28th Annual Conference on Computer Graphics and Interactive Techniques, ser. SIGGRAPH '01. ACM, 2001, pp. 573-580.

[53] A. Hertzmann, "Painterly rendering with curved brush strokes of multiple sizes," in Proceedings of the 25th Annual Conference on Computer Graphics and Interactive Techniques, ser. SIGGRAPH '98. New York, USA: ACM, 1998, pp. 453-460.

[54] - "Non-photorealistic rendering and the science of art," in Proceedings of the 8th International Symposium on Non-Photorealistic Animation and Rendering, ser. NPAR '10. ACM, 2010, pp. 147-157.

[55] A. Hertzmann, C. E. Jacobs, N. Oliver, B. Curless, and D. H. Salesin, "Image analogies," in Proceedings of the 28th Annual Conference on Computer Graphics and Interactive Techniques, ser. SIGGRAPH '01. ACM, 2001, pp. 327-340.

[56] A. Hertzmann and D. Zorin, "Illustrating smooth surfaces," in Proceedings of the 27th Annual Conference on Computer Graphics and Interactive Techniques, ser. SIGGRAPH '00. ACM Press/Addison-Wesley Publishing Co., 2000, pp. $517-526$.

[57] S. Hiller, H. Hellwig, and O. Deussen, "Beyond stippling : Methods for distributing objects on the plane," Computer Graphics Forum, vol. 22, no. 3, pp. 515-522, 2003.

[58] T. Huang, G. Yang, and G. Tang, "A fast two-dimensional median filtering algorithm," IEEE Transactions on Acoustics, Speech, and Signal Processing, vol. 27, no. 1, pp. 13-18, Feb 1979.

[59] T. S. Huang, Two-Dimensional Digital Signal Processing II: Transforms and Median Filters. Berlin, Heidelberg: Springer-Verlag, 1981.

[60] S. Jeschke, "Generalized diffusion curves: An improved vector representation for smooth-shaded images," Computer Graphics Forum, vol. 35, no. 2, pp. 7179, May 2016.

[61] P.-M. Jodoin, E. Epstein, M. Granger-Piché, and V. Ostromoukhov, "Hatching by example: A statistical approach," in Proceedings of the $2 N d$ International Symposium on Non-photorealistic Animation and Rendering, ser. NPAR '02. ACM, 2002, pp. 29-36.

[62] E. Kalogerakis, D. Nowrouzezahrai, S. Breslav, and A. Hertzmann, "Learning hatching for pen-and-ink illustration of surfaces," ACM Trans. Graph., vol. 31, no. 1, pp. 1-17, Feb 2012.

[63] H. Kang, S. Lee, and C. K. Chui, "Coherent line drawing," in Proceedings of the 5th International Symposium on Non-photorealistic Animation and Rendering, ser. NPAR '07. ACM, 2007, pp. 43-50.

[64] — "Flow-based image abstraction," IEEE Transactions on Visualization and Computer Graphics, vol. 15, no. 1, pp. 62-76, 2009. 
[65] M. Kass and J. Solomon, "Smoothed local histogram filters," ACM Trans. Graph., vol. 29, no. 4, pp. 1-10, Jul 2010.

[66] J. Kim and F. Pellacini, "Jigsaw image mosaics," ACM Trans. Graph., vol. 21, no. 3, pp. 657-664, Jul 2002.

[67] S. Y. Kim, R. Maciejewski, T. Isenberg, W. M. Andrews, W. Chen, M. C. Sousa, and D. S. Ebert, "Stippling by example," in Proceedings of the 7th International Symposium on Non-Photorealistic Animation and Rendering, ser. NPAR '09. ACM, 2009, pp. 41-50.

[68] P. Kubelka, "New contributions to the optics of intensely light-scattering materials. part I," J. Opt. Soc. Am., vol. 38, no. 5, pp. 448-457, May 1948.

[69] F. P. Kuhl and C. R. Giardina, "Elliptic Fourier features of a closed contour," Computer Graphics and Image Processing, vol. 18, no. 3, pp. 236-258, 1982.

[70] M. Kuwahara, K. Hachimura, S. Eiho, and M. Kinoshita, Processing of RIAngiocardiographic Images. Boston, MA: Springer US, 1976, pp. 187-202.

[71] J. E. Kyprianidis, "Image and video abstraction by multi-scale anisotropic Kuwahara filtering," in Proceedings of the ACM SIGGRAPH/Eurographics Symposium on Non-Photorealistic Animation and Rendering, ser. NPAR '11. ACM, 2011, pp. 55-64.

[72] J. E. Kyprianidis, J. Collomosse, T. Wang, and T. Isenberg, "State of the "art": A taxonomy of artistic stylization techniques for images and video," IEEE Transactions on Visualization and Computer Graphics, vol. 19, no. 5, pp. 866-885, 2013.

[73] J. E. Kyprianidis and J. Döllner, "Image abstraction by structure adaptive filtering," in Theory and Practice of Computer Graphics, I. S. Lim and W. Tang, Eds. The Eurographics Association, 2008.

[74] J. E. Kyprianidis, H. Kang, and J. Döllner, "Image and video abstraction by anisotropic Kuwahara filtering," Computer Graphics Forum, vol. 28, no. 7, pp. 1955-1963, 2009, special issue on Pacific Graphics 2009.

[75] C. Lee, C. Lee, and C.-S. Kim, "Contrast enhancement based on layered difference representation of 2D histograms," IEEE Transactions on Image Processing, vol. 22, no. 12, pp. 5372-5384, 2013.

[76] A. Levin, D. Lischinski, and Y. Weiss, "Colorization using optimization," in SIGGRAPH'04. New York, NY, USA: ACM, 2004, pp. 689-694.

[77] H. Li and D. Mould, "Contrast-aware halftoning," Comput. Graph. Forum, vol. 29, no. 2, pp. 273-280, 2010.

[78] — - "Structure-preserving stippling by priority-based error diffusion," in Proceedings of Graphics Interface, ser. GI '11. Waterloo, CAN: Canadian HumanComputer Communications Society, 2011, pp. 127-134. 
[79] S. Li, X. Xu, L. Nie, and T.-S. Chua, "Laplacian-steered neural style transfer," in Proceedings of the 25th ACM International Conference on Multimedia, ser. MM '17. ACM, 2017, pp. 1716-1724.

[80] Y. Li, C. Fang, J. Yang, Z. Wang, X. Lu, and M.-H. Yang, "Universal style transfer via feature transforms," in Proceedings of the 31st International Conference on Neural Information Processing Systems, ser. NIPS'17. Curran Associates Inc., 2017, pp. 385-395.

[81] S. Lin, D. Ritchie, M. Fisher, and P. Hanrahan, "Probabilistic color-bynumbers: Suggesting pattern colorizations using factor graphs," ACM Trans. Graph., vol. 32, no. 4, Jul 2013.

[82] Y. Liu, O. Veksler, and O. Juan, "Generating classic mosaics with graph cuts," Computer Graphics Forum, 2010.

[83] S. P. Lloyd, "Least squares quantization in PCM," IEEE Transactions on Information Theory, vol. 28, no. 2, pp. 129-137, 1982.

[84] C. Lu, L. Xu, and J. Jia, "Combining sketch and tone for pencil drawing production," in Proceedings of the Symposium on Non-Photorealistic Animation and Rendering, ser. NPAR '12. Eurographics Association, 2012, pp. 65-73.

[85] J. Lu, S. DiVerdi, W. A. Chen, C. Barnes, and A. Finkelstein, "RealPigment: Paint compositing by example," in NPAR '14, Proceedings of the 12th International Symposium on Non-photorealistic Animation and Rendering. New York, USA: ACM, 2014, pp. 21-30.

[86] D. Martin, C. Fowlkes, D. Tal, and J. Malik, "A database of human segmented natural images and its application to evaluating segmentation algorithms and measuring ecological statistics," in Proc. 8th Int'l Conf. Computer Vision, vol. 2, Jul 2001, pp. 416-423.

[87] D. Martín, G. Arroyo, M. V. Luzón, and T. Isenberg, "Example-based stippling using a scale-dependent grayscale process," in Proceedings of the 8th International Symposium on Non-Photorealistic Animation and Rendering, ser. NPAR '10. ACM, 2010, pp. 51-61.

[88] D. Martín, V. del Sol, C. Romo, and T. Isenberg, "Drawing characteristics for reproducing traditional hand-made stippling," in Proceedings of the Workshop on Non-Photorealistic Animation and Rendering, ser. NPAR '15. Eurographics Association, 2015, pp. 103-115.

[89] J. McCann and N. Pollard, "Soft stacking," Computer Graphics Forum, vol. 31, no. 2, pp. 469-478, May 2012.

[90] M. McGuire, NVIDIA, and W. College, Jul 2010. [Online]. Available: http://stylized.realtimerendering.com/

[91] D. Mould, "A stained glass image filter," in EGRW'03, Proceedings of the 14th Eurographics Workshop on Rendering. Aire-la-Ville, Switzerland: Eurographics Association, 2003, pp. 20-25. 
[92] _ _ "Stipple placement using distance in a weighted graph," in Proceedings of the Third Eurographics Conference on Computational Aesthetics in Graphics, Visualization and Imaging, ser. Computational Aesthetics'07. Eurographics Association, 2007, pp. 45-52.

[93] - "Texture-preserving abstraction," in NPAR '12, Proceedings of the Symposium on Non-Photorealistic Animation and Rendering. Goslar Germany, Germany: Eurographics Association, 2012, pp. 75-82. [Online]. Available: http://dl.acm.org/citation.cfm?id=2330147.2330162

[94] - "Image and video abstraction using cumulative range geodesic filtering," Comput. Graph., vol. 37, no. 5, pp. 413-430, Aug. 2013.

[95] —_ "Authorial subjective evaluation of non-photorealistic images," in Proceedings of the Workshop on Non-Photorealistic Animation and Rendering, ser. NPAR '14. ACM, 2014, pp. 49-56.

[96] D. Mould and K. Grant, "Stylized black and white images from photographs," in Proceedings of the 6th International Symposium on Non-photorealistic Animation and Rendering, ser. NPAR '08. New York, USA: ACM, 2008, pp. $49-58$.

[97] D. Mould and P. L. Rosin, "A benchmark image set for evaluating stylization," in Expressive '16, Proceedings of the Joint Symposium on Computational Aesthetics and Sketch Based Interfaces and Modeling and Non-Photorealistic Animation and Rendering, 2016, pp. 11-20.

[98] L. Neumann and A. Neumann, "Color style transfer techniques using hue, lightness and saturation histogram matching," in Proc. First Eurographics Conference on Computational Aesthetics in Graphics, Visualization and Imaging, ser. Computational Aesthetics'05. Eurographics Association, 2005, pp. 111-122.

[99] P. O’Donovan, A. Agarwala, and A. Hertzmann, "Color compatibility from large datasets," ACM Trans. Graph., vol. 30, no. 4, Jul 2011.

[100] J. Orchard and C. S. Kaplan, "Cut-out image mosaics," in NPAR '08: Proceedings of the 6th international symposium on Non-photorealistic animation and rendering. ACM, 2008, pp. 79-87.

[101] A. Orzan, A. Bousseau, P. Barla, and J. Thollot, "Structure-preserving manipulation of photographs," in NPAR '07, Proceedings of the 5th International Symposium on Non-photorealistic Animation and Rendering. New York, USA: ACM, 2007, pp. 103-110.

[102] A. Orzan, A. Bousseau, H. Winnemöller, P. Barla, J. Thollot, and D. Salesin, "Diffusion curves: A vector representation for smooth-shaded images," Commun. ACM, vol. 56, no. 7, pp. 101-108, Jul 2013.

[103] W.-M. Pang, Y. Qu, T.-T. Wong, D. Cohen-Or, and P.-A. Heng, "Structureaware halftoning," ACM Trans. Graph. (SIGGRAPH 2008 issue), vol. 27, no. 3, pp. 89:1-89:8, 2008. 
[104] G. Papari, N. Petkov, and P. Campisi, "Artistic edge and corner enhancing smoothing," Trans. Img. Proc., vol. 16, no. 10, pp. 2449-2462, Oct 2007.

[105] S. Paris, S. W. Hasinoff, and J. Kautz, "Local Laplacian filters: Edge-aware image processing with a Laplacian pyramid," in ACM SIGGRAPH 2011 Papers, ser. SIGGRAPH '11. ACM, 2011, pp. 1-12.

[106] E. Peli, "Contrast in complex images," J. Opt. Soc. Am. A, vol. 7, no. 10, pp. 2032-2040, Oct 1990.

[107] P. Pérez, M. Gangnet, and A. Blake, "Poisson image editing," ACM Trans. Graph., vol. 22, no. 3, pp. 313-318, Jul 2003.

[108] S. Perreault and P. Hebert, "Median filtering in constant time," IEEE Transactions on Image Processing, vol. 16, no. 9, pp. 2389-2394, Sep 2007.

[109] M. Pollack, "Letter to the editor - the maximum capacity through a network," Operations Research, vol. 8, no. 5, pp. 733-736, Oct 1960.

[110] Y. Qu, T.-T. Wong, and P.-A. Heng, "Manga colorization," ACM Trans. Graph., vol. 25, no. 3, pp. 1214 - 1220, Jul 2006.

[111] E. Reinhard, M. Ashikhmin, B. Gooch, and P. Shirley, "Color transfer between images," IEEE Comput. Graph. Appl., vol. 21, no. 5, pp. 34-41, Sep 2001.

[112] C. Rother, V. Kolmogorov, and A. Blake, "grabcut": Interactive foreground extraction using iterated graph cuts," ACM Trans. Graph., vol. 23, no. 3, p. 309-314, Aug. 2004.

[113] D. L. Ruderman, T. W. Cronin, and C.-C. Chiao, "Statistics of cone responses to natural images: Implications for visual coding," J. Opt. Soc. Am., vol. 15, pp. 2036-2045, 1998.

[114] A. T. Sanda Mahama, A. Dossa, and P. Gouton, "Choice of distance metrics for RGB color image analysis," Electronic Imaging, vol. 2016, pp. 1-4, Feb 2016.

[115] S. Schlechtweg, T. Germer, and T. Strothotte, "RenderBots - multi-agent systems for direct image generation," Computer Graphics Forum, vol. 24, no. 2, pp. 137-148, 2005.

[116] A. Secord, "Weighted Voronoi stippling," in Proceedings of the 2nd International Symposium on Non-Photorealistic Animation and Rendering, ser. NPAR '02. ACM, 2002, pp. 37-43.

[117] A. Semmo, "Design and implementation of non-photorealistic rendering techniques for 3d geospatial data," doctoralthesis, Universität Potsdam, 2016.

[118] A. Semmo, D. Limberger, J. E. Kyprianidis, and J. Döllner, "Image stylization by oil paint filtering using color palettes," in CAE' 15, Proceedings of the Workshop on Computational Aesthetics. Goslar Germany, Germany: Eurographics Association, 2015, pp. 149-158. 
[119] J. Serra, Image Analysis and Mathematical Morphology. USA: Academic Press, Inc., 1983.

[120] — "Connectivity on complete lattices," Journal of Mathematical Imaging and Vision, vol. 9, no. 3, pp. 231-251, 1998.

[121] L. Shapira, A. Shamir, and D. Cohen-Or, "Image appearance exploration by model-based navigation," Comput. Graph. Forum, vol. 28, no. 2, pp. 629-638, 2009 .

[122] M. Shugrina, M. Betke, and J. Collomosse, "Empathic painting: Interactive stylization through observed emotional state," in NPAR '06, Proceedings of the 4 th International Symposium on Non-Photorealistic Animation and Rendering. ACM, 2006, pp. 87-96.

[123] M. Shugrina, J. Lu, and S. DiVerdi, "Playful Palette: An interactive parametric color mixer for artists," ACM Trans. Graph., vol. 36, no. 4, pp. 1-10, Jul 2017.

[124] M. Son, H. Kang, Y. Lee, and S. Lee, "Abstract line drawings from 2D images," in 15th Pacific Conference on Computer Graphics and Applications (PG'07), Oct 2007, pp. 333-342.

[125] Y.-Z. Song, P. L. Rosin, P. M. Hall, and J. Collomosse, "Arty shapes," in Proceedings of the Fourth Eurographics Conference on Computational Aesthetics in Graphics, Visualization and Imaging, ser. Computational Aesthetics'08. Aire-la-Ville, Switzerland: Eurographics Association, 2008, pp. 65-72.

[126] M. Spicker, F. Hahn, T. Lindemeier, D. Saupe, and O. Deussen, "Quantifying visual abstraction quality for stipple drawings," in Proceedings of the Symposium on Non-Photorealistic Animation and Rendering, ser. NPAR '17. ACM, 2017, pp. 1-10.

[127] D. Sýkora, M. Ben-Chen, M. Čadík, B. Whited, and M. Simmons, "TexToons: Practical texture mapping for hand-drawn cartoon animations," in NPAR '11, Proc. ACM SIGGRAPH/Eurographics. New York, NY, USA: ACM, 2011, pp. $75-84$.

[128] D. Sýkora, J. Dingliana, and S. Collins, "LazyBrush: Flexible painting tool for hand-drawn cartoons," Computer Graphics Forum, vol. 28, no. 2, pp. 599-608, 2009.

[129] J. Tan, J.-M. Lien, and Y. Gingold, "Decomposing images into layers via RGBspace geometry," ACM Trans. Graph., vol. 36, no. 4, Nov 2016.

[130] C. Tomasi and R. Manduchi, "Bilateral filtering for gray and color images," in Proceedings of the Sixth International Conference on Computer Vision, ser. ICCV '98. IEEE Computer Society, 1998, pp. 839-846.

[131] A. Vedaldi and S. Soatto, "Quick shift and kernel methods for mode seeking," in Computer Vision - ECCV 2008. Berlin, Heidelberg: Springer Berlin Heidelberg, 2008, pp. 705-718. 
[132] S. Wang, J. Zheng, H. M. Hu, and B. Li, "Naturalness preserved enhancement algorithm for non-uniform illumination images," IEEE Transactions on Image Processing, vol. 22, no. 9, pp. 3538-3548, Sep 2013.

[133] F. Wen, Q. Luan, L. Liang, Y.-Q. Xu, and H.-Y. Shum, "Color sketch generation," in NPAR '06, Proceedings of the 4th International Symposium on Non-photorealistic Animation and Rendering. New York, USA: ACM, 2006, pp. $47-54$.

[134] Wikipedia contributors, "Color difference - Wikipedia, the free encyclopedia," 2019, [Online; accessed 17-September-2019]. [Online]. Available: https: //en.wikipedia.org/w/index.php?title=Color_difference\&oldid=910892104

[135] P. Wilmot, E. Risser, and C. Barnes, "Stable and controllable neural texture synthesis and style transfer using histogram losses," ArXiv, vol. abs/1701.08893, 2017.

[136] B. Wilson and K.-L. Ma, "Rendering complexity in computer-generated penand-ink illustrations," in Proceedings of the 3rd International Symposium on Non-photorealistic Animation and Rendering, ser. NPAR '04. ACM, 2004, pp. $129-137$.

[137] G. Winkenbach and D. H. Salesin, "Computer-generated pen-and-ink illustration," in Proceedings of the 21st annual conference on Computer graphics and interactive techniques - SIGGRAPH'94, 1994, pp. 91-100.

[138] H. Winnemöller, S. C. Olsen, and B. Gooch, "Real-time video abstraction," ACM Trans. Graph., vol. 25, no. 3, pp. 1221-1226, Jul 2006.

[139] H. Winnemöller, J. E. Kyprianidis, and S. C. Olsen, "Xdog: An extended difference-of-gaussians compendium including advanced image stylization." Computers $\&$ Graphics, vol. 36, no. 6, pp. 740-753, 2012.

[140] J. Xu and C. S. Kaplan, "Artistic thresholding," in NPAR '08, Proceedings of the 6th International Symposium on Non-photorealistic Animation and Rendering. New York, USA: ACM, 2008, pp. 39-47.

[141] L. Yatziv and G. Sapiro, "Fast image and video colorization using chrominance blending," Trans. Img. Proc., vol. 15, no. 5, pp. 1120-1129, May 2006.

[142] J. Zander, T. Isenberg, S. Schlechtweg, and T. Strothotte, "High quality hatching," Computer Graphics Forum, 2004.

[143] M. Zhao and S.-C. Zhu, "Sisley the abstract painter," in NPAR '10: Proceedings of the 8th International Symposium on Non-Photorealistic Animation and Rendering. ACM, 2010, pp. 99-107.

[144] M. Zheng, A. Milliez, M. Gross, and R. W. Sumner, "Example-based brushes for coherent stylized renderings," in Proceedings of the Symposium on NonPhotorealistic Animation and Rendering, ser. NPAR '17. New York, USA: ACM, 2017, pp. 1-10. 


\section{Appendix A}

\section{Image Copyright}

Many of the images used in this thesis are provided free for commercial use and modifications permitted. Figure A.1, shows the images come from Benchmark [97] with the names of the image owners.

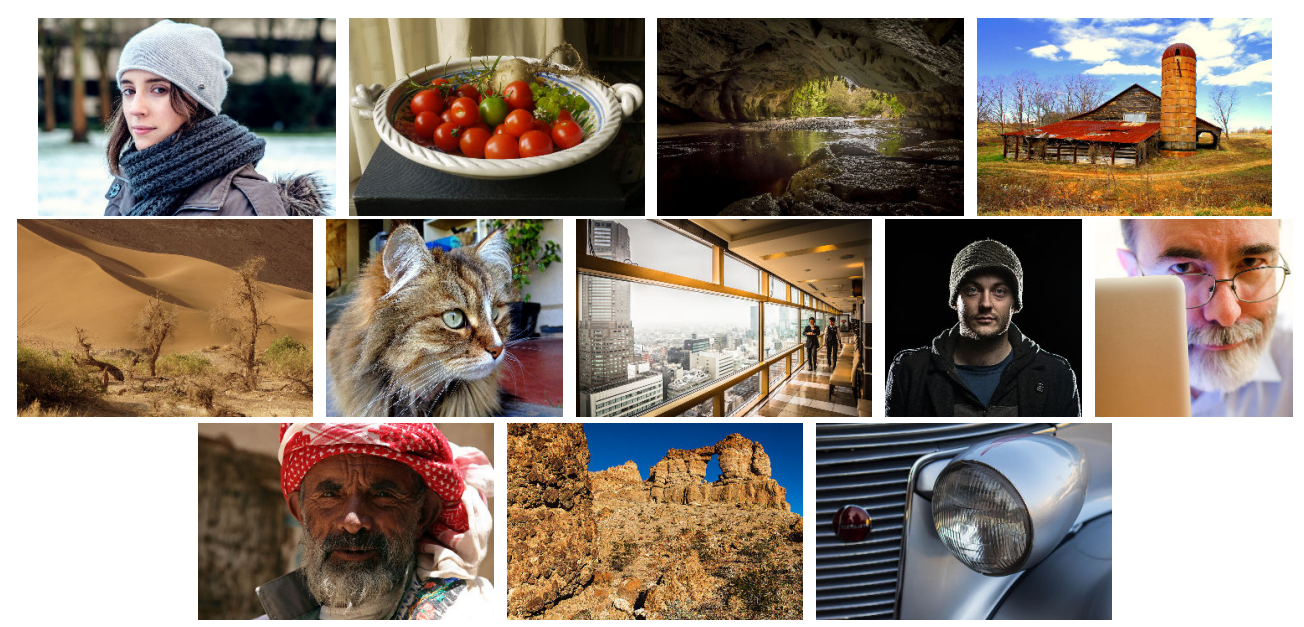

Figure A.1: Benchmark images used in this thesis. Left to right: sicknotepix (toque), Greg Myers (tomatoes), trevorklatko (Oparara), MrClean1982 (barn), Charles Roffey (desert), Theen Moy (cat), Rob Schneider (city), James Marvin Phelps (arch), Paul Stevenson (rim lighting), Martin Kenney (Mac), Richard Messenger (Yemeni), Photos By Clark (headlight).

Figure A.2 shows images "lanterns", "starfish", "lizard", "flower", "old man", "temple", and "boat" from The Berkeley Segmentation Dataset [86].

Figure A.3 shows other Flickr images other than the benchmark images that have been used in this thesis. 

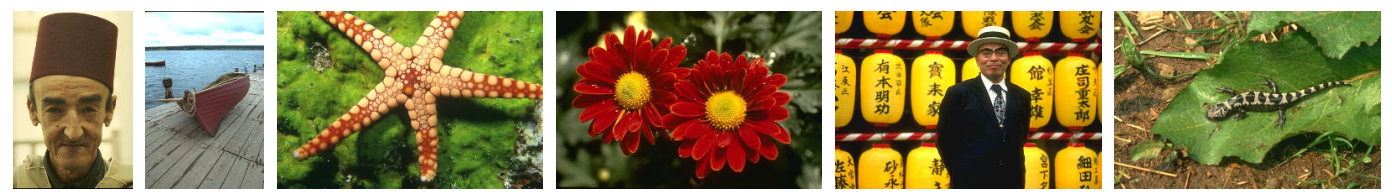

Figure A.2: Images from the Berkeley Segmentation Dataset.
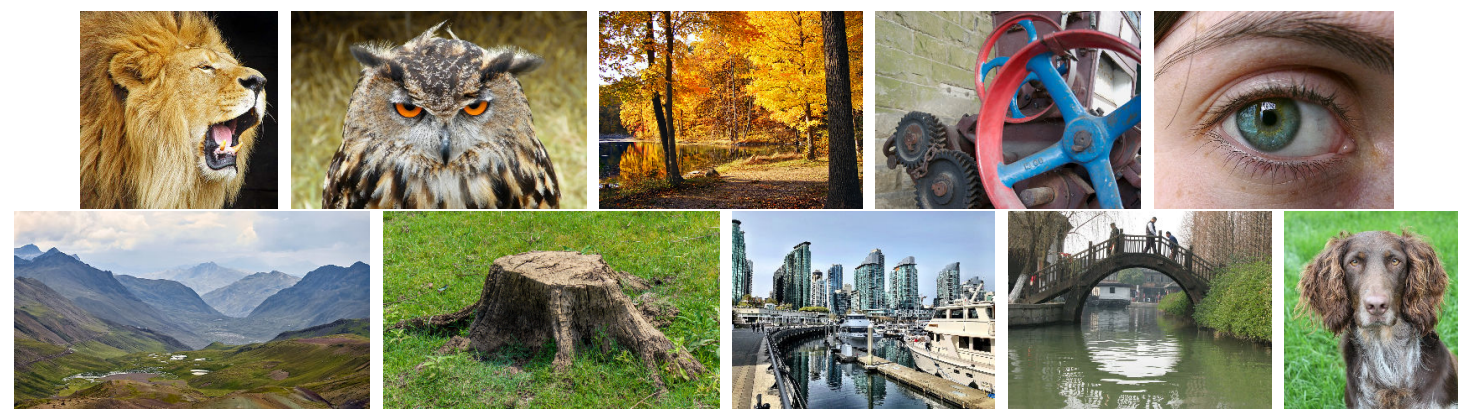

Figure A.3: Tambako the Jaguar (lion), Mark \& Lesley (owl), Nicholas A. Tonelli (autumn), Carl Mueller (machinery), Emi Jane (eye), Alec Gamoff (mountain), Mongkhon Pookpun (stump), David Taylor (port), Carsten Ullrich (bridge), Michal Šcuglík (dog).

The "sketch" and "leaf" images were taken from freesvg.org; the "lion" image is from Vecteezy.com user danvectorman. Market image come from the personal collections of the thesis author.
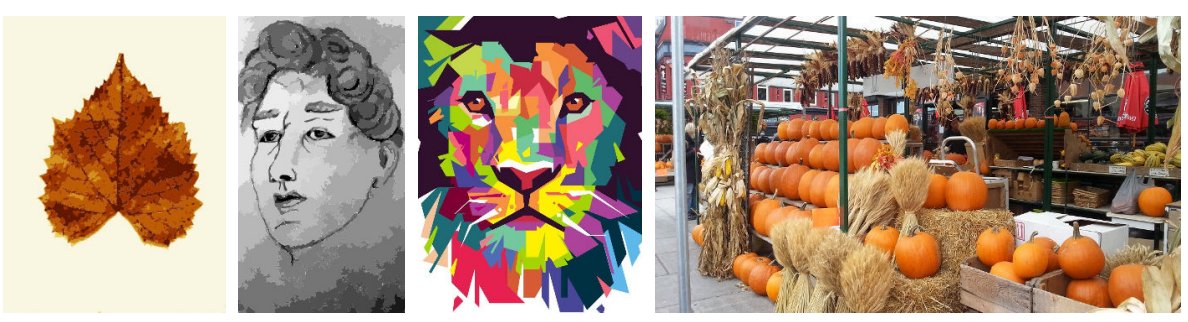

Figure A.4: Vector images: leaf, sketch and lion. Market courtesy of Rosa Azami 